\title{
$21^{\mathrm{e}}$ Congrès Annuel de la Société Française de Toxicologie Analytique (SFTA) \& 51 ${ }^{\mathrm{e}}$ Congrès Annuel de la Société de Toxicologie Clinique (STC)
}

Saint-Malo, 11-14 juin 2013

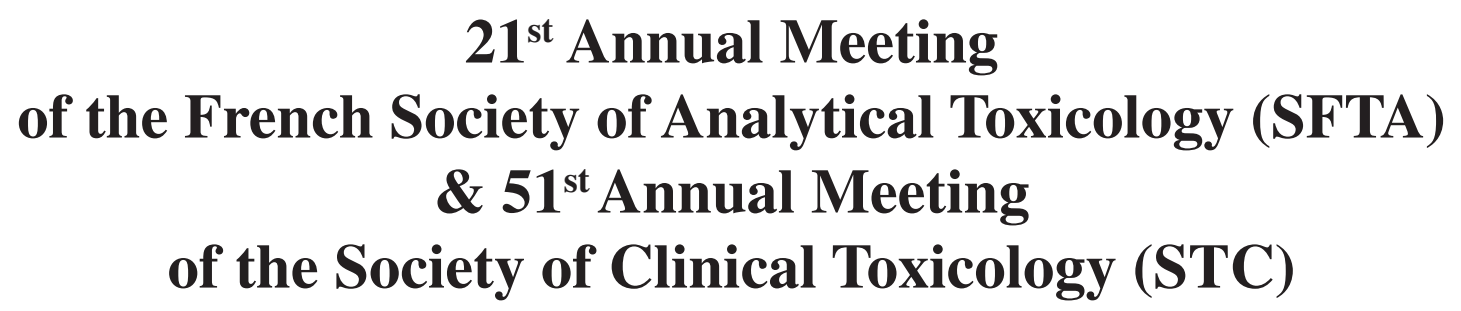

Saint-Malo, June 11-14, 2013

\section{Communications orales}

Risque chimique, Santé au travail, Santé publique ................................................................................. 130

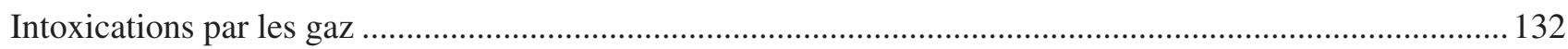

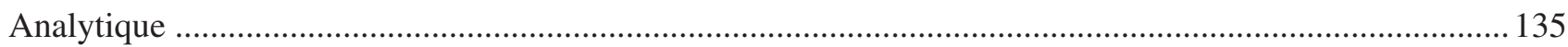

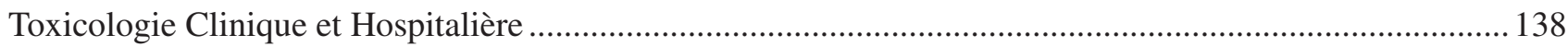

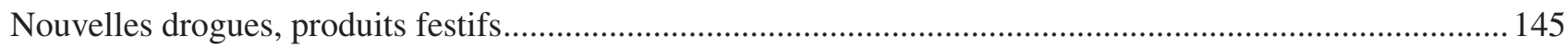

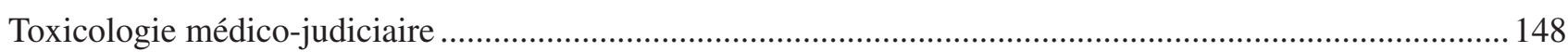

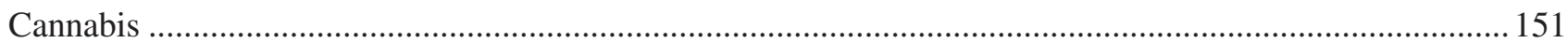

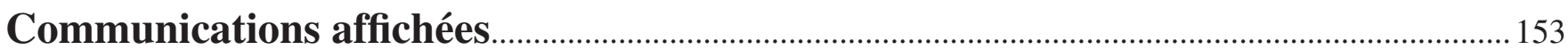




\section{COMMUNICATIONS ORALES}

\section{Risque chimique, Santé au travail, Santé publique}

\author{
C1. Profil métallique par ICP-MS chez l'enfant \\ E Saussereau ${ }^{1}$, P Le Roux ${ }^{1}$, A Cesbron ${ }^{1}$, A Belgaïd ${ }^{1}$, L Mahieu ${ }^{1}$, S Guyet-Job, \\ M Guerbet ${ }^{2}$, JP Goullé $^{1,2}$ \\ ${ }^{I}$ Groupe Hospitalier du Havre, Le Havre; ${ }^{2}$ UFR de Médecine et de \\ Pharmacie, Rouen \\ Objectifs : Les publications concernant les concentrations normales des \\ métaux chez l'enfant sont à la fois peu nombreuses et parfois divergentes. \\ En effet, le recueil du sang, en particulier chez le jeune enfant, constitue \\ une indéniable difficulté. L'emploi d'un plasma à couplage inductif relié \\ à un détecteur de masse (ICP-MS) pour réaliser ces dosages, grâce à une \\ faible prise d'essai, permet de quantifier un grand nombre d'éléments sur un \\ prélèvement sanguin réduit.
}

Matériels et méthodes: Quatre-vingt-dix-neuf enfants des deux sexes (48 âgés de moins de 10 ans, 51 âgés de 10 ans à 18 ans), indemnes de toute affection pouvant affecter le profil métallique, non traités par des oligo-éléments ou des compléments vitaminiques sont inclus, après accord de participation des parents. Un questionnaire comportant des renseignements sur l'habitat, l'environnement familial et scolaire, ainsi que le régime alimentaire, mais également le tabagisme parental accompagne chaque prélèvement. Deux $\mathrm{mL}$ de sang (au minimum $1 \mathrm{~mL}$ ) sont prélevés dans des tubes sous vide en PET de $6 \mathrm{~mL}$ pour éléments traces contenant $10 \mathrm{mg}$ d'EDTA diK (vacutainer réf. 368381, BD diagnostics). L'ICP-MS est un spectromètre X7CCT Thermo Elemental (Thermo Optek), avec cellule de collision réaction (CCR) utilisant comme gaz réactionnel un mélange d'hélium $(93 \%)$ et d'hydrogène $(7 \%)$. L'appareil est équipé d'un passeur d'échantillons et du logiciel PlasmaLab. L'eau employée est purifiée extemporanément sur Synergy UV (Millipore). Les réactifs, de qualité suprapur pour analyses de traces et les solutions étalons de métaux proviennent de chez Merck, Prolabo WWR et Analytika. Les dosages sont réalisés dans le plasma et le sang total $(0,3 \mathrm{~mL})$ après dilution en milieu acide (2,6 $\mathrm{mL}$ de butanol $0,5 \%$ - acide nitrique $1 \%$ - triton $0,01 \%$ pour le plasma et $0,1 \%$ pour le sang - avec 2 étalons internes In et $\mathrm{Rh} 1 \mathrm{ppb}$ ) auxquels on ajoute $0,1 \mathrm{~mL}$ d'acide nitrique à $2 \%$. Pour le plasma, l'étalonnage est effectué en milieu aqueux, alors qu'il est fait appel à la méthode des ajouts dosés pour le sang total. Les éléments suivants sont quantifiés : Li, Be, B, $\mathrm{Al}, \mathrm{V}, \mathrm{Cr}, \mathrm{Mn}, \mathrm{Co}, \mathrm{Ni}, \mathrm{Cu}, \mathrm{Zn}, \mathrm{As}, \mathrm{Se}, \mathrm{Rb}, \mathrm{Sr}, \mathrm{Mo}, \mathrm{Pd}, \mathrm{Ag}, \mathrm{Cd}, \mathrm{Sn}, \mathrm{Sb}, \mathrm{Te}$, $\mathrm{Ba}, \mathrm{W}, \mathrm{Pt}, \mathrm{Hg}, \mathrm{Tl}, \mathrm{Pb}, \mathrm{Bi}$, U. Les dosages de $\mathrm{Cr}$, de $\mathrm{V}$ sont réalisés avec la $\mathrm{CCR}$, selon des procédures déjà décrites. La qualité des résultats obtenus est vérifiée, par des contrôles internes, par la participation au programme canadien de comparaisons interlaboratoires par ICP-MS et par l'emploi de plasma et sang certifiés (Seronorm).

Résultats : Les coefficients de régression sont dans la gamme de linéarité (de 0 à 25 ou $1000 \mathrm{ng} / \mathrm{mL}$ selon les éléments) supérieurs à 0,999 . Les limites de quantification s'échelonnement de moins $1 \mathrm{pg} / \mathrm{mL}$ (U) à 2,9 ng/mL (Zn) pour le sang total, de $2 \mathrm{pg} / \mathrm{mL}$ (U) à $1,6 \mathrm{ng} / \mathrm{mL}$ (Al) pour le plasma. Les concentrations normales sont présentées pour les 30 éléments. Les résultats montrent pour de nombreux métaux des concentrations médianes plus faibles chez les enfants comparativement aux adultes :

- dans le sang total : moins $10 \%$ à moins $40 \%$ pour $\mathrm{Cr}, \mathrm{Co}, \mathrm{As}, \mathrm{Hg}, \mathrm{Pb}, \mathrm{Sr}$, $\mathrm{Ni}, \mathrm{V}, \mathrm{Ag}, \mathrm{Cd}, \mathrm{Sr}, \mathrm{Sn}, \mathrm{Ba}$;

- dans le plasma : moins $10 \%$ à moins $40 \%$ pour $\mathrm{V}, \mathrm{Ni}, \mathrm{As}, \mathrm{Sr}, \mathrm{Ba}, \mathrm{Co}$.
Conclusion : L'ICP-MS est un équipement dont la puissance permet d'établir sur un prélèvement sanguin réduit non seulement le profil métallique chez l'enfant, mais également de mettre en évidence de faibles expositions ou des intoxications à divers éléments tant dans le domaine de la toxicologie clinique que de la toxicologie judiciaire, professionnelle ou environnementale.

$\overline{\text { C2. Ingestion accidentelle d'acétate de plomb : un risque }}$ toujours d'actualité

$\underline{\text { B Lelievre }}^{1}$, M Bretaudeau Deguigne ${ }^{2}$, C Bruneau ${ }^{2}$, C Abbara $^{1}$, S Ferec ${ }^{1}$, P Harry $^{2}$, A Turcant ${ }^{1}$

${ }^{1}$ Laboratoire de pharmacologie et Toxicologie, CHU Angers; ${ }^{2}$ Centre antipoison - Toxicovigilance Angers, CHU Angers

Depuis 1942, la délivrance au grand public de solutions d'acétate de plomb est interdite mais il peut être encore, en 2012, l'objet de prescriptions.

Objectif: Nous décrivons deux observations d'intoxication à l'acétate de plomb. Cas 1: Vers 12h00, un homme de 67 ans ingère accidentellement $250 \mathrm{~mL}$ d'une solution à visée anti-inflammatoire pour usage externe préparée par son kinésithérapeute lui-même et contenant $20 \mathrm{mg} / \mathrm{L}$ d'acétate de plomb (soit une ingestion de $5 \mathrm{mg}$ ) et $20 \mathrm{~g} / \mathrm{L}$ de teinture d'arnica. Sur avis du Centre Anti-Poison, le patient est rapidement transporté aux urgences où il reçoit une irrigation digestive par 6 litres de PEG débutée vers $15 \mathrm{~h}$. Un traitement par du succimer $\left(\right.$ Succicapta $^{\circledR}$ ) à la posologie de $1600 \mathrm{mg} /$ jour pendant 5 jours est instauré le soir même. Un premier prélèvement de sang pour dosage de la plombémie est effectué à $15 \mathrm{~h} 10$. L'examen clinique et la biologie sont parfaitement normaux et aucune opacité digestive n'est visible à l'ASP. Le patient est sorti après $48 \mathrm{~h}$ de surveillance et est resté asymptomatique jusqu'au suivi effectué 90 jours après exposition. Un signalement a été effectué auprès de l'Agence Régionale de Santé pour inspection auprès du kinésithérapeute. Cas 2 : Dans un centre équestre, une jeune femme de 24 ans ingère accidentellement une gorgée de «liqueur de Villate» déconditionnée qui contient $42 \mathrm{~g} / \mathrm{L}$ d'acétate de plomb (associé à de l'acide acétique et du sulfate de cuivre et de zinc). Elle présente 5 minutes après des vomissements, des douleurs abdominales et une douleur de l'oropharynx. Elle est admise aux urgences à H2 où est effectué un premier prélèvement sanguin pour dosage de la plombémie. Une irrigation digestive par 6 litres de PEG et un traitement par Succicaptal ${ }^{\circledR}$ per os sont débutés vers $\mathrm{H} 2$ et $\mathrm{H} 4$ respectivement. Les symptômes se sont rapidement amendés et la patiente est sortie après $24 \mathrm{~h}$ de surveillance. Elle est restée asymptomatique jusqu'au suivi effectué 3 mois après exposition.

Méthodes : Le plomb $\left({ }^{208} \mathrm{~Pb}, \mathrm{E} 1 \mathrm{~V}=7,41 \mathrm{eV}\right)$ est dosé par une méthode validée d'ICP-MS (7500 A, Agilent). L'échantillon sanguin est pré-dilué au 1/2 par une solution de Triton $0,2 \%$ puis dilué au $1 / 20$ dans une solution $\mathrm{d}^{\prime} \mathrm{HNO}_{3} 1 \%$ contenant $10 \mu \mathrm{g} / \mathrm{L}$ de rhénium $\left({ }^{185} \mathrm{Re}, \mathrm{E} 1 \mathrm{~V}=7,87 \mathrm{eV}\right)$ utilisé comme étalon interne. Les contrôles internes de qualité utilisés sont les Seronorm Whole Blood niveau 1 et 2 (Ingen $\left.{ }^{\circledR}\right)$. En outre, le laboratoire participe au programme de comparaisons inter-laboratoires de l'INSP Québec.

Résultats : Pour le patient 1, la plombémie à l'admission (J0, H3) était de $454 \mu \mathrm{g} / \mathrm{L}$. L'absorption digestive de l'acétate de plomb, sel très hydrosoluble, semble donc de l'ordre de $50 \%$. La plombémie a ensuite diminué à $118 \mu \mathrm{g} / \mathrm{L}$ à $\mathrm{J} 2,56,6 \mu \mathrm{g} / \mathrm{L}$ à $\mathrm{J} 5$ et $95,4 \mu \mathrm{g} / \mathrm{L}$ à $\mathrm{J} 14$. Cette cinétique d'élimination rapide confirme une intoxication aiguë. Une plombémie de contrôle faite dans un laboratoire de ville à $\mathrm{J} 90$ était égale à $91 \mu \mathrm{g} / \mathrm{L}$. Pour la patiente 2 , la plombémie de l'admission était de 19,6 $\mu \mathrm{g} / \mathrm{L}$ et la plombémie de contrôle réalisé à $\mathrm{J} 1$ était de $17,9 \mu \mathrm{g} / \mathrm{L}$.

Conclusion : Les intoxications aiguës au plomb par ingestion sont certes peu fréquentes mais nécessitent une prise en charge rapide (antidote et irrigation digestive dans les cas présentés) pour éviter un stockage du plomb dans l'organisme et l'apparition de signes de toxicité. Les doses ingérées, de quelques milligrammes pour le patient 1 , étaient potentiellement très toxiques. Une prise en charge très précoce est recommandée en cas de suspicion d'ingestion de sels de plomb. 


\section{C3. Intoxication aiguë d'un horloger par l'aurocyanure de potassium et d'autres cyanures métalliques}

C Prochalska ${ }^{1}$, B Megarbane ${ }^{2}$, S El Balkhi ${ }^{3}$, J Poupon J ${ }^{3}$, FJ Baud ${ }^{2}$, A Villa ${ }^{1}$, $\underline{\text { R Garnier }}^{1}$

${ }^{1}$ Centre Antipoison, APHP, Groupe hospitalier Lariboisière - St Louis - F Widal, Paris; ${ }^{2}$ Service de réanimation médicale et toxicologique, APHP, Groupe hospitalier Lariboisière - St Louis - F Widal, Paris ; ${ }^{3}$ Laboratoire de toxicologie biologique, Groupe hospitalier Lariboisière - St Louis - F Widal, Paris Des cas d'intoxication par des cyanures métalliques n'ont été que rarement publiés. Nous rapportons un cas d'intoxication par l'aurocyanure de potassium et d'autres cyanures métalliques.

Observation : Un horloger de 69 ans a accidentellement ingéré 10 à $20 \mathrm{~mL}$ d'une préparation contenant $4 \%$ de cyanure de potassium, déconditionnée dans une bouteille d'eau minérale, dans laquelle il avait placé des montres plaquées or, pour les décaper, plusieurs semaines auparavant. Il a présenté des signes d'irritation digestive immédiats, mais les premières manifestations d'une intoxication cyanhydrique (acidose métabolique, hyperlactacidémie et troubles de conscience) ne sont apparues qu' une heure plus tard. Un traitement associant des mesures symptomatiques et l'administration d'hydroxocobalamine a permis la guérison sans séquelle de l'intoxication cyanhydrique.

Méthodes: Des dosages sanguins de cyanure ont été effectués par microdiffusion couplée à la colorimétrie. L'identification et le dosage des métaux impliqués ont été réalisés par ICP/MS dans le liquide ingéré, ainsi que dans le sérum et les urines de l'intéressé.

Résultats: La concentration sanguine de cyanure à l'admission en réanimation était de $3640 \mu \mathrm{g} / \mathrm{L}$, à 270 minutes de l'ingestion et 225 minutes de l'administration de $5 \mathrm{~g}$ d'hydroxocobalamine (population générale [nonfumeurs] : $<100 \mu \mathrm{g} / \mathrm{L}$ ). Du cadmium, du cuivre, du palladium, du zinc et surtout, de l'or et du nickel ont été détectés et mesurés dans la préparation ingérée et dans les liquides biologiques. L'évaluation des concentrations des métaux dans les liquides biologiques a été suivie jusqu'au $6^{\mathrm{e}}$ mois (tableau 1). Il n'a pas été observé de complication systémique de la contamination par ces agents.

Tableau 1 : Concentrations sériques et urinaires des métaux

\begin{tabular}{|l|c|c|c|c|c|c|c|}
\hline Métaux & \multirow{2}{*}{$\begin{array}{c}\text { LOD } \\
(\mu \mathrm{g} / \mathrm{L})\end{array}$} & \multicolumn{3}{|c|}{ Sérum $(\mu \mathrm{g} / \mathrm{L})$} & \multicolumn{3}{c|}{ Urines $(\mu \mathrm{g} / \mathrm{g}$ créatinine $)$} \\
\cline { 3 - 9 } & & $\begin{array}{c}\text { Maximum } \\
(\text { date })\end{array}$ & $\begin{array}{c}\text { Dernier } \\
\text { dosage }\end{array}$ & $\begin{array}{c}\text { Population } \\
\text { générale }\end{array}$ & $\begin{array}{c}\text { Maximum } \\
(\text { date })\end{array}$ & $\begin{array}{c}\text { Dernier } \\
\text { dosage }\end{array}$ & $\begin{array}{c}\text { Population } \\
\text { générale }\end{array}$ \\
\hline Cadmium & 0,0001 & $\begin{array}{c}0,36 \\
(\mathrm{H} 4,5)\end{array}$ & $\begin{array}{c}0,37 \\
(\mathrm{M} 6)\end{array}$ & $<0,40$ & $\begin{array}{c}6,30 \\
(\mathrm{H} 21)\end{array}$ & $\begin{array}{c}0,60 \\
(\mathrm{M} 6)\end{array}$ & $<1,00$ \\
\hline Cuivre & 0,0005 & $\begin{array}{c}1111 \\
(\mathrm{H} 2)\end{array}$ & $\begin{array}{c}908 \\
(\mathrm{M} 6)\end{array}$ & $<1400$ & $\begin{array}{c}209 \\
(\mathrm{H} 7)\end{array}$ & $\begin{array}{c}19 \\
(\mathrm{M} 6)\end{array}$ & $<50$ \\
\hline Nickel & 0,40 & $\begin{array}{c}416 \\
(\mathrm{H} 6)\end{array}$ & $\begin{array}{c}0,7 \\
(\mathrm{M} 6)\end{array}$ & $<1,20$ & $\begin{array}{c}2179 \\
(\mathrm{H} 21)\end{array}$ & $\begin{array}{c}<0,70 \\
(\mathrm{M} 6)\end{array}$ & $<3,80$ \\
\hline Or & 0,40 & $\begin{array}{c}984 \\
(\mathrm{H} 4,5)\end{array}$ & $\begin{array}{c}67 \\
(\mathrm{M} 6)\end{array}$ & $<0,10$ & $\begin{array}{c}113,2 \\
(\mathrm{~J} 22)\end{array}$ & $\begin{array}{c}19,60 \\
(\mathrm{M} 6)\end{array}$ & $<0,25$ \\
\hline Palladium & 0,30 & $\begin{array}{c}20,60 \\
(\mathrm{H} 4,5)\end{array}$ & $\begin{array}{c}1,80 \\
(\mathrm{~J} 22)\end{array}$ & $<0,05$ & $\begin{array}{c}151 \\
(\mathrm{H} 7)\end{array}$ & $\begin{array}{c}1,80 \\
(\mathrm{~J} 22)\end{array}$ & $<0,15$ \\
\hline Zinc & 1 & $\begin{array}{c}3601 \\
(\mathrm{H} 4,5)\end{array}$ & $\begin{array}{c}838 \\
(\mathrm{M} 6)\end{array}$ & $<1300$ & $\begin{array}{c}3440 \\
(\mathrm{H} 7)\end{array}$ & $\begin{array}{c}1025 \\
(\mathrm{M} 6)\end{array}$ & $<1100$ \\
\hline
\end{tabular}

LOD : limite de détection

Discussion : La survenue retardée de l'intoxication cyanhydrique s'explique par la réaction du cyanure de potassium avec les métaux des montres, en particulier avec l'or, pour former des cyanures métalliques dont, après ingestion, l'ion cyanure n'a été que lentement libéré. En l'occurrence, ce délai de survenue a été salvateur, car il a permis une prise en charge médicale efficace de l'intoxiqué. Cette observation est aussi révélatrice de la méconnaissance habituelle par les artisans des dangers et les risques des préparations et des substances qu'ils mettent en œuvre. Dans le cas présent, cette insuffisance de formation et d'information explique qu'un horloger expérimenté ait pu avoir l'habitude de déconditionner une préparation très toxique dans un emballage alimentaire. $\overline{\text { C4. Dosage des métabolites urinaires de la cyperméthrine }}$ dans le cadre de la biométrologie de travailleurs exposés

$\underline{\mathrm{R} L e}$ Grand, G Lachatre, G Lauthier, C Moesch

CHU, Service de Pharmacologie-Toxicologie, Pôle Biologie-Hygiène, Limoges Objectif: La cyperméthrine est un insecticide de la famille des pyréthrinoïdes de synthèse qui agit par contact et ingestion sur un grand nombre d'insectes, à des doses très faibles. Compte tenu du métabolisme rapide et de la courte demi-vie sanguine de la cyperméthrine chez l'Homme, le suivi biométrologique de travailleurs exposés repose sur la quantification de ses métabolites urinaires : 1'acide 3-phénoxybenzoïque (3-PBA) et les acides cis et trans-3-(2,2-dichlorovinyl)-2,2-diméthylcyclopropane-1carboxylique (cis et trans-DCCA). L'objectif de cette étude est de doser ces métabolites dans les urines de personnes exposées travaillant dans une entreprise de fabrication de produits phytosanitaires.

Méthode : Les urines de fin de poste - fin de semaine, de 27 travailleurs exposés, ont fait l'objet d'un dosage. Après une hydrolyse enzymatique, les métabolites ont été extraits par l'hexane en milieu acide puis une purification en milieu alcalin a été réalisée. Les échantillons ont été analysés par CL/SM$\mathrm{SM}$ en mode d'acquisition MRM [m/z $\underline{213} \rightarrow \underline{92,9}$ et $213 \rightarrow 65,1$ (3-PBA); $\mathrm{m} / \mathrm{z} \underline{219} \rightarrow \underline{70}$ (Étalon interne EI : 3-PBA C13); $\mathrm{m} / \mathrm{z} \underline{208,9} \rightarrow \underline{36,9}$ et $\mathrm{m} / \mathrm{z}$ $\underline{207,0} \rightarrow \underline{35}$ (cis et trans-DCCA)].

Résultats: La méthode développée pour ce dosage des métabolites urinaires de la cyperméthrine présente un domaine de mesure qui va de $25 \mathrm{ng} / \mathrm{L}$ à $10000 \mathrm{ng} / \mathrm{L}$, une limite de détection de $15 \mathrm{ng} / \mathrm{L}$ pour chacun des 3 métabolites, des coefficients de variation de reproductibilité, de répétabilité et des biais relatifs inférieurs à $20 \%$. Les résultats de nos dosages ainsi que ceux d'études réalisées chez des travailleurs exposés [Hardt J et al., Int Arch Occup Environ Health. 2003] et dans la population générale [Fréry $\mathrm{N}$ et al., InVS. 2011], sont rapportés dans le tableau suivant :

\begin{tabular}{|l|c|c|c|c|}
\cline { 2 - 5 } \multicolumn{1}{c|}{} & \multicolumn{2}{c|}{ Médiane $(\mathrm{C}$ max $)$} & \multicolumn{2}{c|}{$95^{\mathrm{e}}$ percentile } \\
\cline { 2 - 6 } \multicolumn{1}{c|}{} & Notre étude & $\begin{array}{c}\text { Étude allemande } \\
\text { professionnels } \\
\text { exposés }\end{array}$ & Notre étude & $\begin{array}{c}\text { Étude InVS } \\
\text { population générale }\end{array}$ \\
\hline Analyte & \multicolumn{2}{|c|}{$\mu \mathrm{g} / \mathrm{g}$ de créatinine } & \multicolumn{2}{c|}{$\mu \mathrm{g} / \mathrm{g}$ de créatinine } \\
\hline 3-PBA & $3,27(31,16)$ & $1,40(60,00)$ & 16,14 & $<3,50$ \\
\hline cis-DCCA & $4,39(23,70)$ & $\begin{array}{c}1,80(90,00) \\
\text { (somme cis + trans) }\end{array}$ & 21,39 & $<1,24$ \\
\hline trans-DCCA & $0,35(2,26)$ & & 0,72 & $<2,64$ \\
\hline
\end{tabular}

Conclusion : Dans notre série, les concentrations urinaires des métabolites de la cyperméthrine pour les travailleurs exposés sont nettement plus élevées que celles observées dans la population générale. En terme de médiane, nos valeurs sont légèrement plus élevées que celles rapportées précédemment, mais les concentrations maximales sont plus faibles.

\section{C5. Intoxications oxycarbonées professionnelles en Ile de France (2006 - 2011)}

E Dos Santos, D Chataigner, I Blanc-Brisset, A Villa, R Garnier pour le réseau de surveillance des intoxications oxycarbonées en Ile de France Centre antipoison et de toxicovigilance, Paris

Objectif : Les intoxications professionnelles par le monoxyde de carbone (CO) sont moins fréquentes que celles qui se produisent en milieu domestique. L'objectif de cette étude est de recenser et de décrire les cas survenus sur le lieu de travail, en Ile de France, de 2006 à 2011.

Méthodes : En raison de la fréquence des intoxications oxycarbonées et de leur possible gravité, un réseau de surveillance a été créé. Il associe de nombreux partenaires et a pour objectifs le recueil de tous les cas d'intoxication, le suivi médical des intoxiqués et la réalisation d'enquêtes environnementales pour prévenir les récidives. Des dossiers sont établis par le centre antipoison et de toxicovigilance, pour tous les cas confirmés. Ceux 
qui concernent les accidents survenus en milieu de travail ont été recherchés par un tri manuel.

Résultats : Pendant la période étudiée, 130 accidents sont survenus sur le lieu de travail ; ils concernaient 336 victimes. Le tableau ci-dessous indique le nombre de personnes professionnellement exposées et d'accidents et (entre parenthèse) le nombre total de personnes exposées et d'accidents, toutes circonstances confondues.

\begin{tabular}{|l|c|c|c|c|c|c|c|}
\hline Années & $\mathbf{2 0 0 6}$ & $\mathbf{2 0 0 7}$ & $\mathbf{2 0 0 8}$ & $\mathbf{2 0 0 9}$ & $\mathbf{2 0 1 0}$ & $\mathbf{2 0 1 1}$ & Total \\
\hline Personnes & $38(440)$ & $52(535)$ & $67(571)$ & $55(656)$ & $77(876)$ & $47(635)$ & $336(3713)$ \\
\hline Accidents & $16(206)$ & $19(235)$ & $28(214)$ & $21(212)$ & $26(266)$ & $20(211)$ & $130(1344)$ \\
\hline
\end{tabular}

Les victimes, 111 femmes et 218 hommes, étaient âgées de 15 à 69 ans (médiane: 35 ans). Les principaux secteurs d'activité impliqués étaient le bâtiment (65 exposés [E]; 33 accidents [A]), les locaux de bureaux et assimilés (56 E ; $12 \mathrm{~A})$, le nettoyage (33 E ; $14 \mathrm{~A})$, les entrepôts (31 E ; $8 \mathrm{~A}$ ), les restaurants $(26 \mathrm{E} ; 12 \mathrm{~A})$, la coiffure $(10 \mathrm{E} ; 5 \mathrm{~A})$, les commerces $(10 \mathrm{E}$; $5 \mathrm{~A})$ et la boulangerie $(7 \mathrm{E} ; 6 \mathrm{~A})$. Les sources de $\mathrm{CO}$ les plus souvent en cause étaient les appareils de chauffage (136 E; $51 \mathrm{~A})$, les outils et engins de chantier à moteur $(101 \mathrm{E} ; 36 \mathrm{~A})$, les groupes électrogènes $(61 \mathrm{E} ; 26 \mathrm{~A})$, les matériels de cuisson (18 E; $10 \mathrm{~A})$ et les gaz d'échappement de véhicules (10 E ; 4 A). La symptomatologie est restée inconnue chez 38 personnes $(11 \%)$; dans 83 cas (25\%), aucun symptôme n'est apparu; des signes d'intoxication étaient présents dans 215 cas (64\%). Dans 183 cas sur 215 ( $85 \%$ ) le tableau clinique était bénin, associant des céphalées (152 fois), des sensations vertigineuses ( 87 fois), des nausées et/ou vomissements (67 cas). Ving-sept (27) personnes, 26 hommes et 1 femme, ont eu une perte de connaissance et dans 13 cas la source de $\mathrm{CO}$ était un groupe électrogène ( 9 fois) ou un brasero (4 fois). Deux ouvriers du bâtiment ont été retrouvés morts dans un pavillon en construction dans lequel un groupe électrogène avait été utilisé ; la concentration atmosphérique de CO mesurée par les pompiers était de $1000 \mathrm{ppm}$. Dans 251 cas la prise en charge thérapeutique a comporté l'administration d'oxygène à haute concentration pendant 1 à 12 heures (durée non précisée dans 80 cas); 26 intoxiqués ont bénéficié d'une oxygénothérapie hyperbare.

Conclusion: Les intoxications professionnelles représentent une faible part de l'ensemble des expositions au CO, toutes circonstances confondues ( $<10 \%$ des personnes). Les causes les plus fréquentes sont les outils et engins de chantier à moteur utilisés dans le bâtiment, le nettoyage et le stockage de marchandises et les appareils de chauffage présents dans la plupart des secteurs d'activité. L'emploi inadapté de groupes électrogènes en milieu fermé concerne $10,7 \%$ des personnes exposées, dont deux sont décédées.

\section{Intoxications par les gaz}

\section{$\overline{\text { C6. Les marées vertes : causes, conditions, dangerosité, }}$ prévention}

\section{P Aurousseau}

Conseil Scientifique de l'Environnement de Bretagne (CSEB), Rennes

Introduction: Les marées vertes constituent une des manifestations de l'eutrophisation des eaux marines côtières. Il s'agit d'un phénomène proximal, c'est-à-dire qui se produit proximité immédiate des exutoires d'un certain nombre de fleuves ou de rivières. Dans certaines conditions naturelles favorables, des flux élevés d'azote inorganique peuvent être à l'origine de marées vertes. C'est le cas des rivières et des fleuves de Bretagne qui émettent des flux élevés d'azote nitrique (de 10 à $96 \mathrm{~kg} \mathrm{~N}$ / ha) avec une moyenne régionale de $25 \mathrm{~kg} \mathrm{~N} / \mathrm{ha}$. Les principales conditions naturelles favorables et nécessaires au développement des marées vertes sont un faible renouvellement de l'eau que l'on peut exprimer par le courant « résiduel de marée », des eaux peu profondes, chaudes et assez claires et des sédiments sableux à sablo-limoneux. Parmi les grands sites à marées vertes en Bretagne, on peut citer les baies de Lannion, de Saint-Brieuc, de Douarnenez et de Fouesnant... Parmi des sites qui résistent au phénomène des marées vertes, citons la rade de Brest, la baie du Mont Saint-Michel et l'estuaire de la Vilaine.

Matériel et méthodes : Les algues constitutives des marées vertes sont principalement des ulves. Ces algues sont en effet assez riches en composés soufrés comme tous les organismes vivant en milieu marin, mais elles sont peut-être plus riches en composés soufrés sous forme de sucres sulfatés. En cas d'accumulation sur des épaisseurs assez grandes, les tas d'algues se décomposent en anaérobie. Ceci est dû principalement à leur structure en feuilles qui contribue à la formation de poches qui ne communiquent pas avec l'air ambiant. La décomposition en anaérobie conduit à la production de multiples gaz soufrés au premier rang desquels figure le sulfure d'hydrogène $\mathrm{H}_{2} \mathrm{~S}$. On peut citer de nombreuses autres molécules soufrées : le diméthylsulfure, le méthylmercaptan, le diméthylsulfoxyde... Tous ces gaz ont en commun une forte odeur et sont toxiques comme le sulfure d'hydrogène. La concentration de ce gaz dans les poches peut atteindre 1000 ppm, c'est à dire une teneur largement supérieure au seuil létal. La toxicité du sulfure d'hydrogène est très bien connue dans divers domaines où l'homme peut être exposé à ce gaz. C'est le cas des égouts et du «coup de plomb des égoutiers ». Au-delà d'une certaine concentration la mort est instantanée et l'odeur ne permet plus d'alerter les personnes exposées.

Discussion et conclusion : Toutes ces raisons ont conduit au respect des règles suivantes : les algues doivent être ramassées à l'état frais au plus tard dans les 24 heures qui suivent leur dépôt. L'accès des sites où les algues ne peuvent pas être ramassées doit être interdit avec des marquages appropriés. Le transport des algues doit respecter tout un ensemble de préconisations. Les cabines doivent être équipées de filtres à cartouche, elles doivent être pressurisées. Le personnel doit être équipé de détecteurs. Les détecteurs doivent être approchés des tas d'algues suspects au bout d'une canne. Enfin le traitement des algues par compostage doit être réalisé dans des cuves étanches.

Pour voir disparaître les marées vertes, il faudra descendre les niveaux de concentration en nitrates en dessous 10 voire $5 \mathrm{mg} \mathrm{NO}_{3}^{-} / \mathrm{L}$ ). Si de telles concentrations peuvent sembler basses, elles ne le sont pas pour le milieu marin qui est naturellement un milieu oligotrophe où les teneurs en azote inorganique varient le plus souvent de 0,06 à $0,6 \mathrm{mg} \mathrm{NO}_{3}^{-} / \mathrm{L}$ ). Le domaine des développements des marées vertes correspond à des sites où le taux de dilution des apports d'azote par les fleuves est faible: si l'on part d'eaux douces contenant $60 \mathrm{mg} / \mathrm{L}$ de nitrates, il s'agit d'un domaine où le taux de dilution par l'eau de mer est inférieur à 10 . Si l'on part d'eaux douces contenant $30 \mathrm{mg} / \mathrm{L}$ de nitrates, il s'agit d'un domaine où le taux de dilution par l'eau de mer est inférieur à 5 . C'est la raison pour laquelle ce phénomène peut être appelé «proximal» car de tels taux de dilution ne peuvent s'observer qu'à proximité immédiate des exutoires des fleuves.

\section{C7. Gaz toxiques dans notre environnement}

\section{JP Anger}

Université de Rennes 1

Objectif : Dans les exploitations agricoles, des gaz toxiques peuvent se cacher dans les silos, les fosses à fumier liquide, les cellules à grain et les bâtiments d'élevage. Ces structures offrent des espaces clos dans lesquels certains gaz peuvent s'accumuler et atteindre des concentrations dangereuses. Il est maintenant démontré que la décomposition des algues vertes engendre la formation de gaz extrêmement toxiques, notamment l'hydrogène sulfuré, à des concentrations qui peuvent atteindre des niveaux très importants présentant même un danger mortel par endroits. L'exposition aux fumées d'incendies fait courir aux victimes une pléiade de risques qui 
vont de la chute de l'oxygène dans l'atmosphère ambiante, à l'inhalation de suies chaudes avec des brûlures respiratoires et celle de gaz irritants et asphyxiants. L'inhalation des gaz d'échappement lors du réglage du moteur, ou du travail dans une cabine de péage ou encore de l'utilisation d'un compresseur sur un chantier de construction présente des risques pour la santé des personnels travaillant dans des espaces mal ventilés. Il s'avère donc nécessaire de dresser le bilan des principaux gaz toxiques présents dans de telles ambiances.

Méthode : L'auteur recense les gaz toxiques émis dans l'environnement au cours des processus de fermentation ou de putréfaction des matières organiques animales ou végétales présents dans les fumiers ou les lisiers inhérents aux exploitations agricoles ainsi que ceux produits lors de la décomposition des algues vertes sur les côtes bretonnes. Il s'intéresse également à ceux présents dans les égouts et les stations d'épuration des eaux usées ou enfin ceux formés lors de la combustion de matériaux divers au cours d'incendies ou émis par le pot d'échappement des véhicules automobiles. À cet effet il rappellera leurs caractéristiques physicochimiques et leur toxicité vis à vis de l'homme ainsi que les mesures de prévention à mettre en œuvre pour en limiter les risques au cours d'une exposition accidentelle ou professionnelle.

Résultats : On retrouve dans l'environnement proche des sources précédemment énumérées, un certain nombre de composés gazeux à toxicité variée. Ainsi on peut souvent observer la présence d'oxydes d'azote (NOx): protoxyde d'azote $\left(\mathrm{N}_{2} \mathrm{O}\right)$, oxyde nitreux (NO), oxyde nitrique $\left(\mathrm{NO}_{2}\right)$, de l'ammoniac $\left(\mathrm{NH}_{3}\right)$, des oxydes de carbone : monoxyde de carbone $(\mathrm{CO})$ et dioxyde de carbone $\left(\mathrm{CO}_{2}\right)$, des composés soufrés: hydrogène sulfuré $\left(\mathrm{H}_{2} \mathrm{~S}\right)$ ainsi que des sulfures organiques: methylmercaptan $\left(\mathrm{CH}_{3} \mathrm{SH}\right)$, diméthylsulfure $\left(\mathrm{CH}_{3}\right)_{2} \mathrm{~S}$, diméthylsulfoxyde $\left(\mathrm{CH}_{3}\right)_{2} \mathrm{SO}$, du méthane $\left(\mathrm{CH}_{4}\right)$, et de l'acide cyanhydrique (HCN). Il s'agit là soit de gaz asphyxiants simples ou agissant par un mécanisme biochimique complexe empêchant le transport ou l'utilisation de l'oxygène au niveau cellulaire, soit de gaz irritants responsables de lésions caustiques des muqueuses respiratoires.

Conclusions : Il est évident que l'intervention en milieu professionnel ou l'exposition accidentelle à de telles ambiances toxiques présente un risque vital pour l'individu. Il importe donc en premier lieu de pouvoir identifier et quantifier ces gaz dans les ambiances, d'avertir les sujets exposés, de ventiler les locaux où ils sont susceptibles d'apparaître mais aussi de disposer d'appareils respiratoires autonomes ainsi qu'un harnais de sécurité pour le personnel éventuellement autorisé à intervenir dans de telles ambiances.

\section{C8. Gaz de plongée : théorie et accident(s)}

\section{P Kintz, J-F Garnier}

\section{$X$-Pertise Consulting, Oberhausbergen}

Objectif: La découverte et l'aventure sont inhérentes à l'humanité. La technologie du $21^{\mathrm{e}}$ siècle permet désormais de visiter des régions inaccessibles jusqu'alors. Mais le progrès a un prix. Voilà pourquoi la plongée fait partie des sports d'aventure et des domaines scientifiques ayant connu une forte croissance ces dernières années. La notion de plongée-loisir, développée par l'organisme américain PADI, inclut des exigences précises en matière de limites, de formation et d'équipement. Par définition, le plongeur doit pouvoir continuer à respirer sous l'eau.

Méthode : À partir des données de la littérature, en particulier celle dédiée à la plongée sous-marine et de l'expérience des auteurs (instructeurs OWSI PADI), la théorie de la respiration sous l'eau, les lois régissant les équilibres gazeux et les contraintes des mélanges gazeux, compressés à haute pression seront passées en revue dans cette présentation. Des exemples d'accidents, dans le cadre d'expertises, liés aux gaz respirés permettront de mieux comprendre les dangers potentiels de cette activité sportive.

Résultats : L'air ambiant est essentiellement constitué de $21 \%$ d'oxygène et de $79 \%$ d'azote. Au bord de la mer, la pression atmosphérique est de
1 bar. En plongée, soumise à la pression hydrostatique, la pression de l'eau augmente à un rythme de 1 bar tous les $10 \mathrm{~m}$. Ainsi, à $30 \mathrm{~m}$, la pression ambiante est de 4 bars. Cette pression répondant à la loi de Boyle (Pression $\mathrm{x}$ Volume $=$ constante), le volume pulmonaire s'en trouve affecté.

La loi de Dalton énonce que dans un mélange gazeux, chaque gaz exerce une pression proportionnelle à la pression totale. Dans le cas de l'air, $\mathrm{P}_{\text {total }}=\mathrm{ppO}_{2}$ $+\mathrm{ppN}_{2}$, soit $0,21+0,79$.

C'est l'azote qui est responsable de la narcose en plongée (ivresse des profondeurs) du fait de l'augmentation de la $\mathrm{ppN}_{2}$ au-delà de $30 \mathrm{~m}$. Par ailleurs, l'azote dissous dans le sang pendant la plongée risque de former des bulles (selon la loi de Henry) lors d'une remontée trop rapide ou d'un non-respect des paliers, conduisant au fameux accident de décompression (ADD).

Dans les mélanges enrichis à l'oxygène (NITROX), la proportion d'oxygène peut varier jusqu'à $100 \%$. L'intérêt d'un tel mélange est de diminuer la narcose (puisqu'il y a moins d'azote dans la bouteille), de diminuer l'intervalle de surface entre 2 plongées et d'augmenter le temps de plongée. Par contre, du fait de la toxicité propre de l'oxygène au-delà d'une $\mathrm{ppO}_{2}$ à 1,6 bar, la profondeur maximale doit être ajustée lors de chaque plongée.

Enfin, les applications des mélanges HELIOX (+ hélium), les risques de contamination des blocs de plongées par les gaz d'échappement et donc au monoxyde de carbone et le danger du dioxyde de carbone lors d'un essoufflement seront évoqués.

Conclusion : La plongée sous-marine consiste à explorer le monde sousmarin équipé d'un scaphandre autonome composé d'un gilet stabilisateur, d'un masque, de palmes, d'une bouteille de plongée et d'un détendeur. Ce sport, dont les lois et règles sont différentes selon les pays, est en plein essor. La plongée présente des risques potentiels comme les barotraumatismes et les accidents de désaturation (ADD). Les barotraumatismes sont des accidents touchant les tissus du corps humain, causés par un changement de pression des gaz dans le corps. Ils surviennent lorsqu'il y a une difficulté d'adaptation entre la pression d'air à l'intérieur d'une cavité du corps et la pression externe. Les ADD sont dus à une mauvaise élimination de l'azote restitué par nos tissus, menant à l'apparition de microbulles dans l'organisme et provoquant des lésions multiples.

La complexité des situations et la soudaineté des accidents posent diverses interrogations sur la recherche de l'origine des accidents, mais aussi sur la mise en danger d'autrui par le non respect des règles établies.

\section{C9. L'analyse des gaz dans l'air ambiant}

\section{A Nicolas}

Toxilabo, Nantes

Objectifs : Les analyses de gaz dans l'air répondent à des contextes très variés : évaluation de la qualité de l'air des lieux de travail, de l'air extérieur, intérieur. Différentes méthodes évaluent la pollution de l'air, le risque pouvant être immédiat, subaigu et /ou à plus ou moins long terme. Les risques immédiats sont l'explosion, l'asphyxie, l'intoxication aiguë. Les risques différés concernent en particulier, la cancérogénicité, la reproduction, les maladies chroniques respiratoires, neurologiques. La nuisance aiguë ou chronique correspond à des niveaux d'exposition faibles qu'il est nécessaire de chiffrer afin d'en évaluer le retentissement sur la santé. Les méthodes d'analyses sont choisies pour objectiver le niveau de risque ou pour satisfaire des exigences réglementaires.

Méthodes : Deux stratégies d'analyse sont utilisées: les détecteurs de gaz ou vapeurs à lecture directe, fixes ou portables, et les prélèvements sur adsorbants, dynamiques par pompage, ou passifs par badge, pratiqués pour être ensuite analysés au laboratoire par les méthodes classiques. En milieu industriel, des gaz dangereux sont utilisés soit générés par des procédés ou des déchets. La surveillance des émissions environnementales s'effectue à l'aide de détecteurs fixes. Pour intervenir dans une zone à risque, le personnel s'équipe d'un détecteur portable. Les appareils utilisent le 
principe de l'électrochimie ou de l'absorption infra- rouge. Les composés fréquemment mesurés sont : $\mathrm{CO}, \mathrm{NO}_{2}, \mathrm{SO}_{2}, \mathrm{H}_{2} \mathrm{~S}, \mathrm{HCN}, \mathrm{HCl}, \mathrm{NH}_{3}, \mathrm{Cl}_{2}$ et $\mathrm{CO}_{2}$. Les détecteurs PID, à photo-ionisation, dosent semi-quantitativement de nombreux COV. Les domaines de mesure varient de 0.1 à 10000 ppm, selon les gaz et les objectifs des mesures. Par ailleurs l'hygiène et la santé au travail obligent l'entreprise à des contrôles des niveaux d'exposition individuelle au cours de tâches particulières durant le travail et au cours de l'ensemble du poste. Les contrôles sont validés à partir de prélèvements continus sur adsorbants spécifiques, analysés suivant des méthodes validées, et par le calcul d'une concentration moyenne en fonction du temps de prélèvement. L'interprétation s'effectue par rapport à des valeurs limites (VLCT et VME). Les entreprises dont les activités relèvent d'un arrêté préfectoral ont l'obligation de mesurer les niveaux d'émission en rejet, les mêmes principes de prélèvements et d'analyse sont alors employés. Récemment, l'analyse par détecteurs portables et sur adsorbants s'est élargie au contrôle de la qualité de l'air intérieur, des logements et de certains établissements accueillant du public $\left(\mathrm{CO}_{2}\right.$, formaldéhyde, benzène). Des valeurs guides, VGAI, fixent des recommandations au-delà desquelles des actions sont à entreprendre pour améliorer la qualité de l'air.

Résultats : Nombreux sont les secteurs industriels qui recourent à l'analyse des gaz et vapeurs, dont la pétrochimie, l'industrie pharmaceutique, les ports, les transports, le stockage, le recyclage des déchets; ainsi que les cabinets de contrôles, les services de santé au travail. La qualité des résultats fournis par les détecteurs dépend de leur étalonnage, leur maintenance et des compétences des utilisateurs. Les prélèvements et l'analyse d'adsorbants sont réalisés par des organismes et laboratoires accrédités. Des situations où les concentrations atmosphériques dépassent les valeurs limites, sont décrites telles que la génération de vapeurs d'ammoniac lors de la livraison de matières premières ou durant la fabrication de biscuits, l'émission de formaldéhyde lors d'une synthèse organique utilisant le paraformaldéhyde, le trichlorure d'azote dans l'air des piscines traitées par les composés chlorés, ou encore les teneurs en anesthésiques halogénés dans l'air des blocs opératoires.

Conclusions : Les outils d'analyses de gaz et vapeurs, détecteursenregistreurs à lecture directe, ou l'analyse d'adsorbants en laboratoire, répondent à des besoins différents. Ils contribuent à l'amélioration de la sécurité et de la prévention des risques chimiques des populations, quel que soit leur milieu de vie.

\section{C10. Nouvelles stratégies d'analyse de gaz d'intérêt forensique: de l'échantillonnage à la quantification par étalons internes isotopiques stables (EIIS)}

V Varlet, F Smith, M Augsburger

Unité de Toxicologie et Chimie Forensiques, Centre Universitaire Romand de Médecine Légale, Lausanne, Suisse

Objectif : De nombreux gaz sont responsables chaque année d'intoxications et d'accidents parfois mortels. La stratégie actuelle d'analyse de ces gaz ne suffit plus à assurer une quantification précise pourtant essentielle dans les diagnostics médicolégaux. Notre approche a pour objectif de pallier à ces défauts, depuis l'échantillonnage jusqu'à l'analyse, en présentant les avantages de la quantification par étalons internes isotopiques stables (EIIS) générés in situ.

Méthodes : L'échantillonnage de gaz doit garantir l'étanchéité durant le prélèvement et le stockage des échantillons. Nos protocoles d'échantillonnage permettent de minimiser les fuites lors des prélèvements de gaz et de gaz dissous dans des matrices biologiques. Des informations sont également données quant aux matrices nécessaires selon les gaz suspectés. L'analyse est effectuée par HS-GC-MS/TCD. Un screening des gaz puis un dosage des gaz éventuellement présents sont réalisés grâce à une séparation avec une colonne Select Permanent Gas (tamis moléculaire Q5 Å et CP-Porabond montés en parallèle), permettant de maximiser le nombre de gaz détectables en une seule injection. L'acquisition des signaux MS se fait en mode SIM sur les différents ions moléculaires d'intérêt. La quantification se fait grâce à une calibration interne à partir d'étalons internes isotopiques stables générés in situ (EIIS).

Résultats : L'approche de quantification des gaz par EIIS est documentée par des exemples concrets d'applications forensiques. Par exemple, des étalons internes deutérés sont générés pour la quantification des alcanes volatils (méthane: Varlet V, Augsburger M. Confirmation of natural gas explosion from methane quantification by Headspace-Gas ChromatographyMass Spectrometry in post mortem samples of victims : a case report. International Journal of Legal Medicine. 2013; DOI:10.1007/s00414-0120726-2 1), propane, butane (Varlet V, Smith F, Augsburger M. Accuracy profile validation of a new analytical method for butane measurement using Headspace-Gas Chromatography-Mass Spectrometry. Journal of Chromatography B. 2013; 913-914: 155-160) par l'action de l'eau lourde $\left(\mathrm{D}_{2} \mathrm{O}\right)$ sur le chlorure d'alkyl magnésium (Réactif de Grignard) correspondant. L'emploi d'EIIS a permis d'identifier et renseigner plusieurs cas réels d'explosions mortelles volontaires ou accidentelles en Suisse en 2012. De manière similaire, des étalons internes ${ }^{13} \mathrm{C}$ peuvent être générés pour la quantification du monoxyde de carbone (Varlet V, Lagroy de Croutte E, Augsburger M, Mangin P. Accuracy profile validation of a new method for carbon monoxide measurement in the human blood using Headspace-Gas Chromatography-Mass Spectrometry. Journal of Chromatography B. 2012; 880: 125-131.) et du dioxyde de carbone et ont été utilisés dans plusieurs cas d'intoxications mortelles.

Conclusion : Une nouvelle stratégie d'analyse de gaz d'intérêt forensique est présentée, depuis l'échantillonnage jusqu'à l'analyse. Ses avantages sont comparés aux techniques actuelles notamment en ce qui concerne la quantification par EIIS assurant à la fois la sécurité de l'opérateur et la précision de l'analyse.

\section{C11. Dosage de l'hydrogène sulfuré : bilan de 10 ans d'expérience et contribution du dosage analytique dans l'élucidation de cas d'intoxication chez l'Homme et l'animal}

\section{Cirimele, M Etter, T Wachenheim, M Duez}

\section{Laboratoire ChemTox, Strasbourg}

Objectif : L'intoxication à l'hydrogène sulfuré est une intoxication sévère, souvent mortelle, décrite depuis de nombreuses années en milieu professionnel (industries pétrochimiques, teintureries, fabriques d'engrais...), mais également lors de l'exposition à la décomposition et fermentation anaérobie de substances organiques sous l'action de bactéries (fosses septiques, égouts, fosses à lisier, algues...). Les auteurs rapportent ici plusieurs cas d'intoxication par hydrogène sulfuré survenus chez l'Homme ou l'animal, l'expérience acquise et les dernières améliorations techniques apportées au dosage.

Méthodes : L'échantillon est déposé dans un flacon serti puis de l'acide ortho phosphorique concentré est introduit à travers le septum pour l'étape d'hydrolyse à chaud. Le flacon est alors pressurisé et un aliquote de phase vapeur est transféré au système chromatographique équipé d'une colonne Super-Q plot. La détection est réalisée par un détecteur de masse en mode ionisation par impact électronique et l'acquisition en mode ions sélectionnés. L'identification est basée sur les fragments spécifiques générés $(\mathrm{m} / z, 32 ; 33$ et 34) au temps de rétention spécifique de l'hydrogène sulfuré.

Résultats : Au total, 75 échantillons biologiques (essentiellement sang et poumon) ont été analysés depuis 2003. Les concentrations en hydrogène sulfuré sont rapportées dans le tableau ci-dessous :

\begin{tabular}{|l|c|c|}
\cline { 2 - 3 } \multicolumn{1}{c|}{} & Sang $(\mathrm{mg} / \mathrm{L})$ & Poumon $(\mathrm{mg} / \mathrm{kg})$ \\
\hline Homme & $<0,02$ à $114^{*}$ & $<0,02$ à 1,46 \\
\hline Animaux & $<0,02$ à 0,17 & $<0,02$ à 2,45 \\
\hline
\end{tabular}

*Liquide hématique de rein 


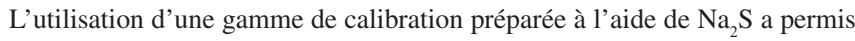
d'augmenter les performances du dosage (précision, reproductibilité, robustesse) par rapport au procédé initial qui faisait appel à la manipulation d'une ampoule de verre chargée en hydrogène sulfuré. Cette nouvelle approche a permis d'étendre la gamme aux concentrations physiologiques $(<0,05 \mathrm{mg} / \mathrm{L})$ et d'abandonner la manipulation de ce gaz très dangereux utilisé comme étalon.

Dans le cadre d'une suspicion d'intoxication par hydrogène sulfuré, il est primordial que les prélèvements biologiques soient rapidement collectés dans des contenants en verre hermétiquement clos et réfrigérés pour procéder à la recherche du gaz dans les plus brefs délais. Pour les décès, il conviendra de procéder au prélèvement de sang et de tissu pulmonaire le plus rapidement possible suite à la conservation du sujet dans des conditions de réfrigération limitant la néoformation de ce gaz. Dans tous les cas, la congélation des prélèvements est recommandée pour leur conservation prolongée.

Pour certains cas d'intoxication mortelle, il a été possible d'observer une valeur particulièrement basse d'hydrogène sulfuré dans le sang alors que le tissu pulmonaire présentait des concentrations élevées. En effet, ce gaz est d'une extrême volatilité, particulièrement instable et de demi-vie courte. De plus, la mise en place de manœuvres de réanimation ou la survenue du décès de façon immédiate du sujet peuvent être à l'origine de cette observation.

Conclusion: L'expérience acquise et les cas rencontrés ont permis de souligner l'intérêt de réaliser le dosage de l'hydrogène sulfuré dans les cas d'intoxication, au niveau sanguin, mais également dans le tissu pulmonaire, site de fixation privilégié de ce gaz hautement toxique. La sulfhémoglobine n'a pas été dosée car sa formation est décrite comme généralement trop faible, et et le dosage n'est pas applicable dans les organes.

\section{Analytique}

\section{C12. L'électrophorèse capillaire en toxicologie en 2013}

\section{P Houzé $^{1}$, L Labat ${ }^{2}$}

${ }^{1}$ Laboratoire de Biochimie, Groupe Saint Louis/Lariboisière/Fernand Widal, AP-HP, Paris ; ${ }^{2}$ Plateforme Biologie du Médicament, Groupe Cochin, APHP, Paris

Objectif : Dans les années 1980, l'apparition du capillaire en silice fondu révolutionnait l'électrophorèse capillaire $(\mathrm{EC})$ et permettait son utilisation en routine dans différents domaines d'applications. En 2001, lors du congrès de la SFTA, J. Hudson (RCM Police, Forensic Laboratory) présentait ses travaux sur un screening de 400 molécules en EC couplée à un détecteur à barrettes de diodes (EC-DBD). Séduisante pour son efficacité, sa rapidité, son faible coût de fonctionnement et sa préparation simplifiée des échantillons, l'EC semblait une méthode prometteuse en toxicologie clinique et médicolégale. Aujourd'hui, à côté d'outils chromatographiques de plus en plus performants comme la LC-MS/MS, il est intéressant de redécrire la place de l'EC dans les domaines de la toxicologie clinique et médico-légale en présentant les dernières applications.

Méthodes : La représentativité de l'EC a été évaluée par une enquête auprès de membres de la SFTA ainsi que par le nombre de publications dans Pubmed entre 2000 et 2012.

Résultats : Au travers du sondage effectué au sein de la SFTA, 4 laboratoires rapportent l'utilisation d'une EC avec détection UV-Visible (EC-UV/VIS) ou DBD. Deux utilisent encore l'EC en « routine» pour des applications toxico-biologiques. Deux autres ont utilisé l'EC mais n'ont pas poursuivi avec cette méthodologie (comparaison EC et LC-UV-Remedi pour le screening toxicologique; dosage des inhibiteurs de la recapture de la sérotonine et de leurs métabolites en toxicologie médico-légale).
D'après une analyse Pubmed, plus de 2500 publications impliquent les méthodes LC-MS/MS en toxicologie clinique et médico-légale entre 2000 et 2012, contre 196 pour l'EC durant la même période. Seule l'équipe de Tagliaro publie régulièrement des méthodes de screening toxicologique dans le sang, les urines ou les cheveux mais avec un couplage EC-MS (TOF, Maldi TOF). Malgré une sensibilité souvent insuffisante, l'EC-UV/VIS peut se révéler complémentaire des méthodes chromatographiques en particulier pour le dosage des ions inorganiques (thiosulfate), organiques (acides issus du métabolisme des alcools toxiques), des marqueurs d'exposition (CDT), de certains médicaments très polaires (pralidoxime) ou encore des composés difficilement quantifiables par d'autres méthodes (diméthylsulfoxide).

Conclusion : Temps d'analyse court et préparation d'échantillon simplifiée, atouts initiaux de l'EC sont aujourd'hui retrouvés dans les méthodes utilisant les systèmes LC-MS/MS dont la sensibilité est très supérieure. En attendant un couplage aisé et fiable, type «plug and play » avec la spectrométrie de masse, l'EC peut encore trouver sa place dans les laboratoires de toxicologie clinique et médico-légale pour des applications ponctuelles.

\section{C13. Intoxication à l'alcool frelaté ou soins de conservation ? L'électrophorèse capillaire à la rescousse !}

\section{Dumestre-Toulet ${ }^{1}, \mathrm{P}$ Houzé $^{2}, \mathrm{~N}$ Grosleron ${ }^{3}, \mathrm{~S}_{\text {Garapon }}{ }^{3}$}

${ }^{1}$ Laboratoire Toxgen, Bordeaux ; ${ }^{2}$ Laboratoire de Biochimie, Groupe Saint Louis/Lariboisière/Fernand Widal, AP-HP, Paris; ${ }^{3}$ Unité de Médecine Légale, CHU Pellegrin, Bordeaux

Objectif : Un Girondin de 44 ans décède à l'hôpital de l'île de Lombok, Indonésie, plusieurs heures après avoir absorbé un cocktail alcoolisé artisanal. La possibilité d'une intoxication avec de l'alcool frelaté, déjà décrite sur l'île de Bali voisine, est très vite évoquée par les amis et la famille. Le corps est rapatrié à Bordeaux deux semaines après, non sans avoir subi des soins de conservation. Après autopsie, des analyses toxicologiques pour recherche des causes de la mort sont requises par le Parquet. Elles mettent en évidence des taux élevés de méthanol. Une technique est alors développée en électrophorèse capillaire de zone pour doser l'acide formique (sous la forme ionisée formates), métabolite du méthanol dans différents milieux biologiques. La présence de formates dans les prélèvements pourrait en effet indiquer une métabolisation du méthanol et étayer l'hypothèse d'intoxication méthanolique.

Méthodes : Les analyses toxicologiques ont été réalisées selon les techniques du laboratoire dans le cadre des RCM et les alcools ont été dosés par HS/GC/ FID dans différents fluides prélevés sur le corps avec le 2-butanol comme étalon interne. L'électrophorèse capillaire de zone est réalisée avec une détection en UV inverse à $254 \mathrm{~nm}$. La technique est linéaire de 0,015 à $4 \mathrm{~g} / \mathrm{L}$, la précision et l'exactitude sont inférieures à $11 \%$ et la LOD est fixée à $0,005 \mathrm{~g} / \mathrm{L}$, le succinate est utilisé comme étalon interne. La méthode permet également la quantification d'autres anions organiques (lactates, acétates) et inorganiques (chlorures, sulfates phosphates). La recherche d'interférences a été effectuée pour différentes anticoagulants : citrate, EDTA et héparine.

Résultats : Les analyses de recherche de stupéfiants et de médicaments effectuées par LC/DAD, GC/MS et LC/MS-MS sont négatives. Les résultats des dosages d'alcools et de formates effectués selon les méthodes précitées sont détaillés dans le tableau suivant :

\begin{tabular}{|l|c|c|c|c|c|}
\cline { 2 - 6 } \multicolumn{1}{c|}{} & Urines & Humeur vitrée & $\begin{array}{c}\text { Contenu } \\
\text { gastrique }\end{array}$ & bile & $\begin{array}{c}\text { Liquide cavité } \\
\text { abdominale }\end{array}$ \\
\hline éthanol & $0,11 \mathrm{~g} / \mathrm{L}$ & $0,39 \mathrm{~g} / \mathrm{L}$ & $0,27 \mathrm{~g} / \mathrm{L}$ & $0,13 \mathrm{~g} / \mathrm{L}$ & $0,34 \mathrm{~g} / \mathrm{L}$ \\
\hline méthanol & $2,40 \mathrm{~g} / \mathrm{L}$ & $1 \mathrm{~g} / \mathrm{L}$ & $5,10 \mathrm{~g} / \mathrm{L}$ & $2,25 \mathrm{~g} / \mathrm{L}$ & $4,06 \mathrm{~g} / \mathrm{L}$ \\
\hline formates & $2,50 \mathrm{~g} / \mathrm{L}$ & $0,48 \mathrm{~g} / \mathrm{L}$ & & $0,42 \mathrm{~g} / \mathrm{L}$ & $0,73 \mathrm{~g} / \mathrm{L}$ \\
\hline
\end{tabular}

Les dosages de formates ont été également effectués dans des liquides de thanatopraxie utilisés en France, ainsi que dans le formol utilisé en anatomopathologie. Les résultats sont respectivement de $<0,015 \mathrm{~g} / \mathrm{L}$ et de $0,052 \mathrm{~g} / \mathrm{L}$. Le dosage de formol n'a pas été effectué. Il n'a pas été possible 
de récupérer le liquide utilisé pour embaumer le corps de la victime. D'après les données de la littérature (Viinamäki et al., Forensic Sci Int. 2011), les concentrations de formates urinaires sont supérieures à $2 \mathrm{~g} / \mathrm{L}$ dans $90 \%$ des intoxications méthanoliques avérées. Les concentrations mesurées chez la victime sont donc compatibles avec une intoxication méthanolique même si on ne peut exclure une contamination par le liquide de conservation indonésien dont seule une analyse permettrait de lever le doute.

Conclusion : Il n'est pas possible de conclure aujourd'hui pour notre cas où nous sommes toujours en attente du liquide de conservation utilisé et pour lequel une demande officielle a été effectuée par l'ambassade de France en Indonésie. La technique d'électrophorèse capillaire apporte néanmoins une aide précieuse à l'expertise toxicologique de recherche des causes de la mort en permettant la recherche et la quantification des formates, métabolites qui caractérisent une intoxication au méthanol.

\section{C14. Dépistage des drogues dans le plasma par immunoessai CEDIA sur l'analyseur Indiko Plus}

A Verstraete, M de Sloovere, M Hollebosch, E van Caeneghem, V Stove Laboratoire de biologie clinique, Hôpital Universitaire, Gent, Belgique

Objectif : Les laboratoires de toxicologie clinique reçoivent souvent du plasma pour la recherche des drogues. Les techniques de référence comme la chromatographie en phase gazeuse ou liquide couplée à la spectrométrie de masse (CG-SM et CL-SM) sont très sensibles et spécifiques, mais elles ne sont pas disponibles $24 \mathrm{~h}$ sur 24,7 jours sur 7 . Nous avons adapté les tests CEDIA existants sur l'analyseur Indiko Plus (Thermo Fisher Scientific, Vantaa, Finlande) pour l'analyse du plasma.

Méthodes : Nous avons utilisé les méthodes CEDIA pour le fluide oral pour l'amphétamine et la méthamphétamine et les tests CEDIA urinaires pour le cannabis, la cocaïne et les opiacés, en modifiant certains paramètres comme le volume de l'échantillon ou des réactifs. Les réactifs, contrôles et calibrateurs ont été gracieusement mis à disposition par Thermo Fisher Scientific. Nous les avons comparés à nos méthodes de dépistage existantes : pour l'amphétamine et la méthamphétamine la CL-SM/SM et pour les opiacés, la cocaïne et les cannabinoïdes par des tests urinaires EMIT modifiés, qualitatifs, sur le Dimension Xpand (Siemens). Les dépistages positifs par la technique EMIT (et $10 \%$ des négatifs) ont été confirmés par CG-SM. Les limites de quantification de la CG-SM étaient de $1 \mu \mathrm{g} / \mathrm{L}$ pour le tétrahydrocannabinol et le 11-hydroxy- $\Delta 9$-tétrahydrocannabinol, $5 \mu \mathrm{g} / \mathrm{L}$ pour l'acide 11-nor- $\Delta$ 9-tétrahydrocannabinol-9-carboxylique et $10 \mu \mathrm{g} / \mathrm{L}$ pour les autres substances. Des restants de plasma anonymisés ont été utilisés pour les analyses. Pour les analyses CEDIA, des calibrations à 4 points par dilution automatique ont été effectuées. Deux niveaux de contrôles (dilués manuellement) ont été utilisés. Les seuils de dépistage optimaux ont été déterminés par les courbes ROC (receiver operating characteristic). Tous les échantillons analysés pour l'amphétamine ont également été analysés pour la méthamphétamine.

Résultats : Pour tous les contrôles, la répétabilité était $<4 \%$ pour tous les tests. L'imprécision sur 20 jours était de 5,9\% $(20 \mu \mathrm{g} / \mathrm{L})$ et 3,8 \% (40 $\mu \mathrm{g} / \mathrm{L})$ pour l'amphétamine, $10,6 \mu \mathrm{g} / \mathrm{L}(9,6 \mu \mathrm{g} / \mathrm{L})$ et $5,5 \%(20,8 \mu \mathrm{g} / \mathrm{L})$ pour les cannabinoïdes, $4,9 \%(24,77 \mu \mathrm{g} / \mathrm{L})$ et $4,4 \%(43,44 \mu \mathrm{g} / \mathrm{L})$ pour la cocaïne, $6,7 \%(22,5 \mu \mathrm{g} / \mathrm{L})$ et $8,9 \%(37,5 \mu \mathrm{g} / \mathrm{L})$ pour la méthamphétamine et $8,9 \%$ $(22,5 \mu \mathrm{g} / \mathrm{L})$ et $5,2 \%(37,5 \mu \mathrm{g} / \mathrm{L})$ pour les opiacés. Les seuils de dépistage des méthodes CEDIA étaient : $9 \mu \mathrm{g} / \mathrm{L}$ pour l'amphétamine ( $\mathrm{n}=58), 10 \mu \mathrm{g} / \mathrm{L}$ pour les cannabinoïdes $(\mathrm{n}=70), 15 \mu \mathrm{g} / \mathrm{L}$ pour la cocaïne $(\mathrm{n}=65), 23 \mu \mathrm{g} / \mathrm{L}$ pour la méthamphétamine $(\mathrm{n}=58)$ et $13 \mu \mathrm{g} / \mathrm{L}$ pour les opiacés $(\mathrm{n}=61)$. Avec ces seuils, nous avons obtenu une séparation complète des positifs et des négatifs (100\% de sensibilité et spécificité) pour tous les paramètres à l'exception de l'amphétamine, où il y avait 1 faux positif et 1 faux négatif, ce qui correspond à une sensibilité de $94 \%$ et une spécificité de $98 \%$.

Conclusions : Les tests CEDIA adaptés peuvent détecter les drogues dans le plasma de façon très sensible, rapide et fiable.

\section{C15. Développement de deux méthodes pour l'analyse de composés organiques volatils, de pesticides et des métabolites associés dans le méconium}

\author{
M Meyer-Monath $^{1}$, I Morel ${ }^{2}, \mathrm{~K} \mathrm{Tack}^{1}$, F Lestremau ${ }^{1}$ \\ ${ }^{1}$ INERIS, Direction des Risques Chroniques, Verneuil-en-Halatte; \\ ${ }^{2}$ Laboratoire de Toxicologie Biologique et Médico-légale, CHU Pontchaillou, \\ Rennes
}

Objectif: Le méconium est une matrice complexe analysée afin de déterminer si le degré d'exposition des fœtus aux xénobiotiques joue un rôle dans la survenue de malformations, ceci dans le cadre du projet PHRC «PENEW» (Pregnancy Environment and NEWborn malformations, PHRC 2010, CHU Rennes). Le développement analytique pour la détection et le dosage dans le méconium de BTEX (benzène, toluène, éthylbenzène, xylènes), de deux solvants trichlorés (trichloroéthylène et tétrachloroéthylène) issus de la famille des COVs (Composés Organiques Volatils), de pesticides (pyréthrinoïdes, organophosphorés...) et des métabolites associés est présenté dans ce travail.

Méthodes : La préparation d'échantillon est complexe en raison des propriétés physico-chimiques très particulières du méconium. Concernant les COVs, la technique par MicroExtraction par Phase Solide (MEPS) en espace de tête couplée à une CG/SM a été choisie car elle ne nécessite pas de préparation préalable de l'échantillon et permet de le préserver pour une autre analyse (utile lors de faibles quantités collectées). L'analyse des métabolites de COVs, des pesticides et des métabolites de pesticides est ainsi réalisée sur le même échantillon par une extraction sur phase solide (SPE). Des cartouches de phases adsorbantes diverses (phase inverse, échange d'anions, échange de cations...) ont été évaluées. Les conditions de séparation des composés cibles ont été optimisées par le test de cinq colonnes d'interactions différentes (phase inverse, HILIC, mode mixte), par la variation des tampons aqueux pour l'analyse CL/SM/SM (nature et quantité du sel, $\mathrm{pH}$ ) et par l'optimisation du gradient chromatographique.

Résultats : La colonne Rtx-WAX (30 m × $0.25 \mathrm{~mm}$ ID., épaisseur de film de $0.50 \mu \mathrm{m}$ ) utilisée permet une séparation de tous les COVs étudiés en particulier les isomères du xylène. L'optimisation de la méthode par MEPS/CG/SM a montré que la fibre CAR/PDMS $85 \mu \mathrm{m}$ produisait les meilleures sensibilités et que l'ajout de sel n'était pas nécessaire. Les paramètres d'extraction ont été choisis en considérant le meilleur compromis entre la sensibilité spectrométrique et le temps d'analyse. Les essais ont démontré qu'une calibration interne dans le méconium avec des étalons internes spécifiques de type isotopique pour chaque composé analysé était indispensable pour une quantification fiable dans cette matrice. Des limites de quantification (LQ) de l'ordre de 100 pg/g de méconium sont atteintes par cette méthode.

Pour l'analyse par SPE/CL/SM/SM, les phases présentant les meilleurs rendements sont celles de type phase inverse (C18 polymérique) et échange d'anions forts (SAX). L'utilisation de trois colonnes d'interactions différentes pour la quantification est nécessaire pour l'analyse de ces molécules aux propriétés physico-chimiques très différentes: la RP-Amide (phase inverse) pour l'analyse des molécules apolaires et moyennement polaires ; la HILIC (phase normale) pour les molécules polaires et très polaires ; et la Trinity P1 (mode mixte) pour le glyphosate. L'optimisation des conditions chromatographiques permet 1'utilisation des mêmes solvants pour ces trois colonnes. Des LQ de l'ordre de $10 \mathrm{ng} / \mathrm{g}$ de méconium sont obtenues avec cette méthode.

Conclusion : Grâce à ces deux méthodes analytiques, basées sur l'utilisation du même échantillon contenant une faible quantité de méconium $(1,25 \mathrm{~g})$, il est possible de quantifier la quarantaine de molécules cibles et de mettre ainsi en évidence une exposition du fœtus aux contaminants de l'environnement. 


\section{C16. Intérêt de l'acquisition $\mathrm{MS}^{3}$ apportée par la technologie QTRAP : dosage simultané du $\Delta^{9}$-THC, du CBN, du CBD et du $\Delta^{9}$-THC-COOH dans les cheveux par CL-SM/SM}

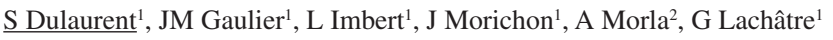

${ }^{I}$ Service de Pharmacologie, Toxicologie et Pharmacovigilance, CHU, Limoges ; ${ }^{2} A B$ Sciex - Courtabcuf

Objectif : Dans le domaine des analyses capillaires, le diagnostic d'une consommation de chanvre indien repose sur la recherche et/ou le dosage de 4 marqueurs : $\Delta^{9}$-THC, cannabinol (CBN), cannabidiol (CBD) et $\Delta^{9}$-THC$\mathrm{COOH}$. La mise en évidence d'une consommation chronique de chanvre indien ne pose généralement pas de problème. En revanche, dans la plupart des autres situations (exposition d'enfants au chanvre indien, suivi de sevrage...), seule la présence du dérivé carboxylique du $\Delta^{9}$-THC permet de différencier une consommation d'une exposition passive (Uhl et al., Forensic Sci Int. 2004). La plupart du temps, l'analyse de ces marqueurs fait appel à la chromatographie en phase gazeuse couplée à la spectrométrie de masse simple (analyse du $\Delta^{9}$-THC, du CBD et du CBN) (Musshoff et al., Forensic Sci Int. 2003) et en tandem (CG-SM/SM) (analyse du $\Delta^{9}$-THC-COOH) (Uhl et al., Forensic Sci Int. 2004). Nous présentons ici une méthode originale permettant le dosage simultané (extraction, séparation et détection) de ces 4 marqueurs par chromatographie en phase liquide couplée à la spectrométrie de masse en tandem (CL-SM/SM).

Méthodes : Après ajout des étalons internes $\left(\Delta^{9}-\mathrm{THC}-\mathrm{D}_{3}\right.$ et $\Delta^{9}$-THC$\mathrm{COOH}-\mathrm{D}_{3}$ ) et hydrolyse des cheveux par de la soude à chaud, les composés d'intérêt sont extraits à $\mathrm{pH}$ acide par un mélange acétate d'éthyle / éther diéthylique. Ils sont ensuite séparés par une colonne Waters Atlantis T3, puis détectés à l' aide d'un API 5500 QTRAP (AB Sciex). Deux modes de balayage sont utilisés : MRM pour le $\Delta^{9}$-THC $(313,1 \rightarrow \underline{245,1}$ et 190,8$)$, le $\Delta^{9}$-THC-D $(316,1 \rightarrow 248,2)$, le CBD $(313,0 \rightarrow \underline{245,1}$ et 106,9$)$ et le CBN $(309,0 \rightarrow \underline{279,1}$ et 221,9$)$, ainsi que $\mathrm{MS}^{3}$ pour le $\Delta^{9}$-THC-COOH $(343,1 \rightarrow 299,2 \rightarrow 245,2)$ et le $\Delta^{9}$-THC-COOH-D $(346,1 \rightarrow 302,2 \rightarrow 248,2)$.

Résultats : La méthode de dosage a été validée selon une procédure de validation évaluée favorablement par le CoFrAc. Les critères de validation définis dans cette procédure ont été remplis pour les 4 analytes. Les limites de détection et de quantification sont du même ordre de grandeur que celles obtenues pour les techniques de CG-SM et CG-SM/SM et sont compatibles avec les dernières recommandations de la Society of Hair Testing (Cooper et al., Forensic Sci Int. 2012). Ainsi la limite de quantification du $\Delta^{9}$-THC$\mathrm{COOH}$ est de $0,2 \mathrm{pg} / \mathrm{mg}$. Elle est de $50 \mathrm{pg} / \mathrm{mg}$ pour le THC, le CBD et le CBN. Les épreuves de répétabilité et de fidélité intermédiaire ont respectivement montré des $\mathrm{CV}$ inférieurs à $14 \%$ et $15 \%$; des biais inférieurs à $11 \%$ et $9 \%$, pour les 4 molécules.

Conclusion : Au-delà de ses performances, cette méthode de dosage simultané (une seule extraction et un seul passage chromatographique) par CL-SM/SM des 4 marqueurs capillaires d'une exposition au cannabis présente 3 avantages: (i) en toxicologie biologique et médico-légale, le champ des applications d'un système de CL-SM/SM, tel que l'API 5500 QTRAP, est beaucoup plus étendu que celui d'un système de CG-SM/SM. (ii) il n'y a pas d'étape de dérivation contrairement aux méthodes de dosage du $\Delta^{9}$-THC-COOH par CG-SM/SM, (iii) cette méthode s'avère efficiente quelle que soit la situation.

\section{C17. Étude de la stabilité des taches de sang sur différents supports}

C Jamey, A Tracqui, B Ludes, JS Raul

Laboratoire de Toxicologie, Institut de Médecine Légale, Strasbourg

Introduction : En pharmacologie, l'analyse des gouttes de sang séché est de plus en plus employée notamment pour les suivis thérapeutiques et les études pharmacocinétiques. La conservation de l'échantillon est simplifiée avec l'utilisation de cartes papier-filtre. En toxicologie médico-légale, une nouvelle approche analytique est envisagée avec le recueil de traces de sang sur les scènes de crime. Dans cette optique, nous avons étudié la stabilité de certains xénobiotiques (bromazépam, diazépam, zolpidem, méthadone, cyamémazine et tramadol) pour une concentration de $100 \mathrm{ng} / \mathrm{mL}$.

Méthode : Afin de reproduire des conditions réelles, les gouttes de sang ont été déposées sur différents supports (verre, carrelage, papier peint et tissus) puis exposées à différentes conditions d'environnement (lumière et obscurité) et de durée ( $\mathrm{J} 0, \mathrm{~J}+1, \mathrm{~J}+3, \mathrm{~J}+1$ semaine et $\mathrm{J}+1$ mois). Après une extraction en phase liquide, l'analyse a été réalisée par chromatographie liquide ultra haute performance couplée à la spectrométrie de masse en tandem (UPLC-MS/MS). Des gouttes de sang de $25 \mu \mathrm{L}$, surchargées à $100 \mathrm{ng} / \mathrm{mL}$ des molécules à tester, sont déposées sur les différents supports puis exposées aux conditions environnementales choisies. Le sang séché est ensuite prélevé avec un écouvillon imprégné d'eau distillée (verre, carrelage et papier-peint), ou découpé (papier peint et tissus). L'échantillon est ensuite réhydraté pendant une heure et demie avec un tampon ammonium à pH 9,5. Une extraction en phase liquide en présence de diazépam-d5 (standard interne) est alors réalisée. L'analyse est effectuée ensuite par UPLC-MS/MS. La validation de la méthode a été réalisée sur des gouttes de sang déposées directement sur des écouvillons, du papier-peint et du tissus.

Résultats : Dans ces conditions analytiques, la méthode s'avère être sensible (LOQ : $2 \mathrm{ng} / \mathrm{mL})$, linéaire (LOQ $-5000 \mathrm{ng} / \mathrm{mL})$ et répétable $(\mathrm{CV}<20 \%)$. Les rendements d'extraction sont supérieurs à $35 \%$ et aucun effet matrice significatif n'est constaté. Toutes les molécules testées ont été détectées. Aucune dégradation majeure n'a été relevée : on observe des diminutions de concentration de l'ordre de 20 à $30 \%$ selon les molécules et les supports. Conclusion : Cette étude permet de démontrer la stabilité de certains xénobiotiques sur différents supports, sans influence majeure du délai d'exposition et des conditions environnementales. Le recueil des taches de sang par écouvillons ou découpage offre une nouvelle approche analytique en toxicologie médico-légale susceptible de présenter un intérêt sur certaines scènes de crime (absence de cadavre, traces de sang provenant d'un agresseur en fuite, etc.).

$\overline{\text { C18. Criblage par CLUHP-SM à haute résolution dans le }}$ sang, de cinquante-deux composés organiques d'origine naturelle d'intérêt toxicologique

J Carlier $^{1}$, J Guitton ${ }^{2}$, F Bevalot ${ }^{3}$, L Fanton ${ }^{4}$, Y Gaillard ${ }^{1}$

${ }^{1}$ Laboratoire LAT-LUMTOX, La Voulte-sur-Rhône; ${ }^{2}$ Laboratoire de toxicologie, faculté de pharmacie, Lyon; ${ }^{3}$ Laboratoire LAT-LUMTOX,

Lyon ; ${ }^{4}$ Institut de Médecine Légale, faculté de médecine, Lyon

Objectif : Le monde végétal et animal est une riche source de nourriture et le fondement des médecines traditionnelles à travers le monde. Ils sont également une arme puissante et une source importante de substances pharmacologiquement actives. En conséquence, les intoxications d'origine naturelle ne sont pas rares, que la cause soit accidentelle (usage détourné, méconnaissance des espèces, mauvais dosage, mauvaise préparation), à visée suicidaire ou criminelle.

Les méthodes d'analyse multirésidus et les criblages dans les échantillons biologiques sont de bons outils de détermination d'une intoxication, en particulier dans le cas d'échantillons de volume réduit. Nous présentons ici une méthode de criblage dans le sang, orientée vers l'identification et la quantification de cinquante-deux composés naturels d'intérêt toxicologique : l'aconitine, l'ajmaline, l' $\alpha$-amanitine, l' $\alpha$-lobéline, l' $\alpha$-solanine, l'anabasine, l'atropine, la baccatine III, la brucine, la bufoténine, la céphalomannine, la cévadine (non quantifiée), la cévine (non quantifiée), la colchicine, la coniine, la convallatoxine, la cymarine, la cytisine, la digitoxine, la digoxine, l'émétine, la gelsémine, l'harmine, l'ibogaïne, la jervine, la kavaïne, le lanatoside C, la lupanine, la méthysticine, la mitragynine, la monocrotaline, la N,Ndiméthyltryptamine, la nériifoline, l'oléandrine, l'ouabaïne, le paclitaxel, la pilocarpine, la podophyllotoxine, la proscillaridine A, la psilocine, la quinine, 
la réserpine, la rétrorsine, la ricinine, la salvinorine A, la scopolamine, la sénécionine, la spartéine, la strophanthidine, la strychnine, la vératridine, et la yohimbine.

Méthodes : Les analyses sont réalisées en chromatographie en phase liquide à ultra-haute performance couplée à une détection par spectrométrie de masse (CLUHP-SM) à haute résolution (technologie orbitrap). Il s'agit d'un criblage large de $m / z 120$ à 1050 en mode positif. La quantification est réalisée en mode SIM (selected ion monitoring) à \pm 5 ppm de la masse exacte des composés. L'identification des molécules est ensuite confirmée lors d'une seconde injection en CLUHP-SM ${ }^{2}$ par leur spectre de fragmentation. La préparation de l'échantillon consiste en une extraction en phase solide (EPS) sur cartouche Oasis ${ }^{\circledR}$ HLB (Waters ; Guyancourt, France).

Résultats : La méthode a été validée pour cinquante molécules dans le sang total, pour des concentrations s'échelonnant de 0,1 à 500 ng. $\mathrm{mL}^{-1}$. Les limites de détection et de quantification étaient basses, atteignant la dizaine de $\mathrm{pg} \cdot \mathrm{mL}^{-1}$. Le rendement d'extraction variait selon les composés de 35 à $100 \%$.

Conclusion: La détection à haute résolution permet de réaliser une quantification hautement spécifique en mode SIM en combinant le niveau de sensibilité de la $\mathrm{SM}^{2}$ et la flexibilité du criblage. La spécificité de la haute résolution permet en outre de valider cette méthode de criblage.

Il s'agit à notre connaissance, de la première méthode de dosage dans le sang d'un nombre aussi important de toxiques d'origine naturelle.

\section{C19. Automatisation de la recherche large de xénobiotiques par le couplage SPE online-UPLC-QToF}

A Rifflet $^{1}$, C Richeval $^{1}$, L Humbert $^{1}$, M Lhermitte $^{1}$, G Tournel $^{2}$, D Allorge ${ }^{1,3}$ ${ }^{1}$ Laboratoire de Toxicologie et Génopathies, Centre de Biologie et Pathologies, CHRU, Lille; ${ }^{2}$ Institut de Médecine Légale, CHRU, Lille; ${ }^{3}$ Faculté de Pharmacie, Université Nord de France, Lille

Contexte: L'extraction de matrices biologiques complexes (sang total, sérum, bile...) est une étape décisive dans l'identification des xénobiotiques dans le cadre d'un criblage toxicologique. De nombreuses approches, décrites dans la littérature, font appel à des extractions liquide-liquide avec des solvants organiques variés, ou à des extractions en phase solide (SPE), voire encore à de simples précipitations (Jang et al., J Pharm Biomed Anal. 2013 ; Johnson and Botch, J Anal Toxicol. 2011 ; Roman et al., Anal Bioanal Chem. 2013). Les extractions liquide-liquide présentent cependant quelques inconvénients majeurs : elles sont chronophages, parfois opérateurdépendantes et généralement favorables à certains types de molécules. L'extraction SPE offre l'avantage d'être facilement automatisable, soit par l'emploi d'automates d'extraction (offline), soit par un couplage direct avec le système chromatographique (online).

Objectif : Au laboratoire, deux extractions liquide-liquide, une sous condition basique et l'autre en acide, sont actuellement menées en parallèle pour cette recherche (Richeval et al., Ann Toxicol Anal. 2011). Le but de ce travail a été d'automatiser la recherche large de xénobiotiques en développant une méthode générique d'extraction pour le couplage UPLCQToF. Les résultats obtenus en routine seront comparés avec ceux issus de la méthode développée.

Matériel et Méthode : $100 \mu \mathrm{L}$ de sang total ou d'humeur vitrée, ainsi que $20 \mu \mathrm{L}$ d'urine, de bile ou de contenu gastrique, auxquels sont ajoutés $100 \mu \mathrm{L}$ d'étalon interne (solution méthanolique de méthylclonazépam et de $\beta-\mathrm{OH}$ éthylthéophylline), sont déprotéinisés par de l'acide sulfosalicylique à $3 \%$. Après centrifugation, $100 \mu \mathrm{L}$ de surnageant sont injectés sur une colonne SPE Oasis ${ }^{\circledR}$ HLB $(20 \mu \mathrm{m}, 2.1 \times 30 \mathrm{~mm})$ connectée, par le biais de vannes, à une colonne analytique Acquity UPLC ${ }^{\circledR}$ HSS C18 $(1,8 \mu \mathrm{m}, 2,1 \times 150 \mathrm{~mm})$. L'échantillon est chargé sur la colonne SPE sous un débit de $2 \mathrm{~mL} / \mathrm{min}$ d'eau contenant $2 \%$ d'ammoniaque. L'élution par «backflush» et la séparation chromatographique sont réalisées à l'aide d'un gradient tampon formate d'ammonium $\mathrm{pH} 3$ / acétonitrile à $0,1 \%$ d'acide formique. L'acquisition en electrospray positif est réalisée en mode $\mathrm{MS}^{\mathrm{e}}$ et le traitement des données est managé par le logiciel POSIT \pm VE.

Résultats : L'analyse des résultats, effectuée sur une centaine d'échantillons de cas réels, démontre l'efficacité de notre système pour l'extraction d'un plus grand nombre de xénobiotiques dans la recherche des causes de la mort ou d'intoxications. Contrairement à notre procédure actuelle, l'extraction online a permis d'isoler et d'identifier des molécules très polaires comme la metformine. Ce protocole permet de détecter à la fois des molécules basiques, neutres et acides en mode electrospray positif. L'association entre l'extraction SPE online, l'acquisition $\mathrm{MS}^{\mathrm{e}}$ et la base de données POSIT $\pm \mathrm{VE}$ contribuent à l'amélioration du criblage toxicologique.

Conclusion : L'automatisation du criblage toxicologique par couplage SPE online-UPLC-QTof réduit considérablement le temps de préparation des échantillons, ainsi que le temps d'acquisition. Après un simple prétraitement de l'échantillon, la SPE online apporte de la robustesse au criblage toxicologique.

\section{Toxicologie Clinique et Hospitalière}

\section{$\overline{\text { C20. Anamnèse, clinique, biologie : une trilogie souvent }}$ imparfaite}

F Flesch $^{1}$, AM Gutbub ${ }^{2}$, L Berthelon ${ }^{1}$, P Sauder $^{3}$, S Gayol $^{4}$

${ }^{1}$ Centre antipoison et de toxicovigilance, $\mathrm{CHU}$, Strasbourg; ${ }^{2}$ Service de réanimation, Hôpitaux Civils, Colmar ; ${ }^{3}$ Service de réanimation médicale, Nouvel Hôpital Civil, Strasbourg ; ${ }^{4}$ Service des urgences, Nouvel Hôpital Civil, Strasbourg

Introduction : La prise en charge de patients pour intoxications aiguës fait partie de l'activité quotidienne des services d'urgence et plus rarement des services de réanimation. La trilogie anamnèse, examen clinique, examens biologiques, peut être prise en défaut du fait de patients peu coopérants ou comateux à l'admission ou de médecins trop occupés pour percevoir la discrète altération clinique et/ou biologique qui va être déterminante pour orienter la suite des investigations et la prise en charge du patient.

Objectif : Illustrer, à l'aide de cas cliniques, les pièges et difficultés du diagnostic toxicologique et l'importance de la collaboration clinicoanalytique.

Méthode : 5 cas d'intoxication, dont 4 médicamenteux et 1 non médicamenteux sont rapportés. Pour chacun de ces cas sont retracés précisément l'anamnèse, les antécédents, le tableau clinique et biologique ainsi que l'évolution. Chacun de ces cas illustre une particularité soit anamnestique, soit clinique, soit biologique susceptible de perturber l'évaluation initiale du risque: antécédents qui peuvent «égarer» l'évaluation de la gravité potentielle de l'intoxication, anamnèse inexistante ou erronée, tableau clinique discordant par rapport à l'anamnèse, absence de contrôle des paramètres biologiques. Seule l'analyse toxicologique permet alors, dans ces cas, de confirmer le diagnostic et d'adapter le traitement.

Conclusion: La prise en charge d'un patient intoxiqué implique une anamnèse précise, un examen clinique, un examen biologique ainsi qu'une analyse toxicologique le cas échéant. Lorsqu'il existe une discordance entre certains de ces paramètres, le dialogue entre le clinicien et l'analyste est alors indispensable pour confirmer l'intoxication et adapter la thérapeutique. Disposer d'un prélèvement sanguin initial permet, dans certains cas, de faire un diagnostic à posteriori et de rectifier la prise en charge médicale. 


\section{C21. Toxidrome «cholinergique » : apport de l'analyse toxicologique pour optimiser l'utilisation précoce d'antidote aux urgences}

\section{$\underline{\text { P Nisse }}^{1}$, JM Gaulier ${ }^{2}$, AM Guerin-Robardey ${ }^{3}$, A Garat ${ }^{1,4}$}

${ }^{1}$ Centre antipoison et toxicovigilance, Lille; ${ }^{2}$ Laboratoire de toxicologie, Limoges ; Réanimation Polyvalente, Beauvais ; ${ }^{4}$ Laboratoire de toxicologie, Lille

Objectif : Lors d'une intoxication volontaire par un produit phytopharmaceutique déconditionné et non étiqueté, la clinique, même si elle est évocatrice, n'est pas toujours suffisante pour différencier le chlormequat, un herbicide, d'un insectide organophosphoré ou d'un carbamate anticholinestérasique. Ainsi, devant un toxidrome «cholinergique», l'identification précoce de la substance phytopharmaceutique est parfois nécessaire pour une administration optimale des antidotes et éviter l'éventuelle survenue de complications.

Cas clinique : Un homme de 73 ans ingère volontairement une solution rose de préparation artisanale dite «à but rodenticide», sans autre indice sur la composition. Très rapidement, le patient présente une altération de la conscience, des mouvements anormaux incontrôlés, une diarrhée, une incontinence urinaire et une hypersalivation. L'hémodynamique est stable. Le patient est intubé, ventilé et sédaté (par des morphiniques). En réanimation, on note un myosis et la persistance de la diarrhée, de l'hypersialorrhée et d'un encombrement bronchique. Le traitement est symptomatique. Un prélèvement de la substance absorbée est adressé au laboratoire de toxicologie. À J+3, le diagnostic d'intoxication aux insecticides organophosphorés est posé devant l'identification du mévinphos dans la préparation et l'effondrement de l'activité des acétylcholinestérases érythrocytaires et sériques. Un traitement par oximes est alors initié et poursuivi durant 24 heures. À J+4, alors que la sédation est levée et que les signes cholinergiques ont quasi disparu, le tableau clinique se complique par la survenue d'un syndrome intermédiaire nécessitant une réintubation du patient.

Discussion : Même si le syndrome intermédiaire reste une complication rare des intoxications aux organophosphorés, sa gravité, du fait de l'atteinte respiratoire, justifie une surveillance d'au moins 96 heures en réanimation. Compte tenu de l'effondrement de l'activité de l'acétylcholinestérase mesuré au moment de l'apparition de ce syndrome et persistant pendant toute sa durée, plusieurs hypothèses ont été proposées quant à son origine et notamment une inadéquation du traitement par les oximes ou un retard dans leur utilisation. En effet, le syndrome intermédiaire est probablement lié à un excès persistant d'acétylcholine au niveau de la plaque motrice. Les oximes peuvent lever l'inhibition enzymatique tant que les acétylcholinestérases inhibées n'ont pas encore subi le processus de vieillissement irréversible. Pour être totalement efficace, les oximes doivent être administrées dans les 8 premières heures si l'on veut espérer une lever du blocage neuromusculaire (et en tout état de cause avant la $36^{\mathrm{e}}$ heure où intervient le vieillissement des cholinestérases phosphorylées). Si la mesure de l'activité des acétylcholinestérases permet de différencier une intoxication au chlorméquat (activité normale) d'une intoxication aux anticholinestérasiques (effondrée), elle ne permet pas faire la différence entre un carbamate insecticide et un organophosphoré (activité effondrée dans les 2 cas). Seule l'identification précoce de l'insecticide organophosphoré permettra une administration à bonne dose et sans délai des oximes afin de lever le blocage neuromusculaire, de régénérer les cholinestérases et probablement de prévenir et d'éviter la survenue d'un syndrome intermédiaire.

Conclusion : Si la confirmation analytique en urgence ne doit pas être systématique lors de tentative d'autolyse par ingestion de pesticides formellement identifiés (en dehors du paraquat), la caractérisation analytique devient indispensable pour la prise en charge d'une intoxication volontaire par un pesticide non identifié.
C22. Les dosages biologiques et toxicologiques dans l'intoxication volontaire aiguë par antidépresseurs sont-ils pertinents ?

N Franchitto, F Pelissier

Centre antipoison et de toxicovigilance, Centre Hospitalier Universitaire Purpan, Toulouse

Objectif : En France, les intoxications médicamenteuses volontaires (IMV) ont une incidence annuelle de 4/1000 habitants. La prise en charge hospitalière associe la prescription de bilans biologiques et toxicologiques. Le but de notre étude était d'évaluer les indications de ces bilans dans l'intoxication volontaire aiguë par antidépresseurs, après la réactualisation des recommandations de pratiques professionnelles sur le sujet.

Méthodes : Nous avons réalisé une étude observationnelle, rétrospective, de 191 IMV par antidépresseurs, seuls ou avec d'autres toxiques, admises dans un service d'urgences du CHU entre janvier et octobre 2012. Les données socio-démographiques des patients, la dose toxique supposée ingérée, les bilans biologiques et toxicologiques ont été recueillis et analysés.

Résultats : 84,3\% des patients avaient ingéré des ISRS, et 15,7\% des antidépresseurs tricycliques (ATD). L'âge médian du premier groupe était de 40 ans (sex-ratio H/F : 0,4), et de 43,5 ans pour le deuxième (sex-ratio : 0,7). $55,5 \%$ des patients avaient associé une prise de benzodiazépines, 27,7\% de 1'alcool, et $14,1 \%$ les deux. Des analyses toxicologiques qualitatifs et/ou quantitatifs ont été effectués pour $77 \%$ des patients (77\% des IMV aux ISRS et 76,7\% de celles aux ATD). Dans les cas d'IMV aux ATD, leur présence a été détectée dans $52,4 \%$ des cas. Un bilan électrolytique sanguin a été prescrit pour 93,3\% des IMV aux ATD, et pour 90,1\% des IMV aux ISRS. Une numération formule sanguine a été effectuée pour $93,3 \%$ des IMV aux ATD, et pour $88,2 \%$ de celles aux ISRS. Les bilans hépatiques étaient plus fréquents pour les IMV aux ATD que pour les autres $(p=0,03)$. Aucune relation statistiquement significative n'a été retrouvée entre la prise d'autres toxiques (alcool, benzodiazépines) ou la médicalisation du transport, et la réalisation de bilans biologiques et toxicologiques. La natrémie moyenne des IMV aux ISRS était mesurée à $140 \mathrm{mmol} / \mathrm{L}$ et celle des IMV aux ATD à $138,9 \mathrm{mmol} / \mathrm{L}(\mathrm{p}=0,03)$. Aucun autre désordre électrolytique (kaliémie, réserve alcaline) n'a été noté.

Conclusion : La réactualisation des recommandations pour la prescription des examens de biologie dans le cadre des intoxications graves a pour but d'uniformiser les pratiques dans les prescriptions de bilans toxicologiques. Pour les intoxications aux antidépresseurs, la quantification du toxique dans le sang ou les urines est rarement corrélée à l'intensité du tableau clinique. L'objectif de l'analyse qualitatif permet l'orientation diagnostique dans les cas suspects d'overdose ou lorsque le patient est inconscient au moment de la prise en charge. Les bilans électrolytiques sanguins doivent être orientés en fonction des antécédents du patient et des co-ingestions associées.

\section{C23. Surveillance des expositions aux antihistaminiques de $2^{\mathrm{e}}$ génération chez l'enfant}

M Bretaudeau Deguigne, E Rouxel, C Bruneau, D Boels, P Harry

Centre antipoison - toxicovigilance, $C H U$ Angers

Objectif : L'objectif de cette étude est de faire une mise au point sur la toxicité des antihistaminiques de $2^{\mathrm{e}}$ génération dans un contexte de surdosage aigu chez l'enfant et de proposer des recommandations de prise en charge en fonction des doses supposées ingérées (DSI).

Méthode : Étude rétrospective et observationnelle des mono expositions chez l'enfant de moins de 15 ans à un antihistaminique de $2^{\mathrm{e}}$ génération (cétirizine, lévocétirizine, loratadine, desloratadine, féxofenadine, ébastine et mizolastine) enregistrées dans les systèmes d'information du CAPTV

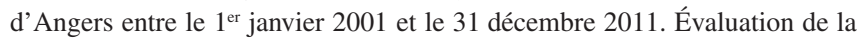
gravité des intoxications selon le Poisoning Severity Score (PSS) (Persson et 
al., J Toxicol Clin Toxicol. 1998). La comparaison des valeurs quantitatives a été effectuée par test de Mann et Whitney.

Résultats : Au total 603 expositions chez l'enfant ont été recensées dont 36 expositions volontaires. L'âge moyen était de $4 \pm 3,6$ ans ( 1 mois 15 ans). Près de $95 \%$ des enfants sont restés asymptomatiques $(n=571)$. Pour les 32 enfants symptomatiques, les symptômes observés étaient une somnolence $(n=14)$, des vomissements $(n=6)$, une agitation $(n=4)$, une tachycardie $(n=3)$, des céphalées $(n=3)$, des nausées $(n=1)$, des douleurs digestives $(n=1)$, une sécheresse des muqueuses $(n=1)$ et des vertiges $(n=1)$. La gravité globale était toujours faible (PSS 1). Un total de 130 enfants a consulté aux urgences et 49 ont reçu du charbon activé. Aucune anomalie de 1'ECG n'a été observée. La DSI était renseignée dans 569 dossiers. La DSI moyenne pour les enfants symptomatiques et asymptomatiques ainsi que les DSI maximales sans effet clinique observé sont présentées dans le tableau ci-dessous.

\begin{tabular}{|c|c|c|c|c|}
\hline & PSS & $\begin{array}{c}\text { DSI moyenne } \\
(\mathrm{mg} / \mathrm{kg})+/-\mathrm{SD}\end{array}$ & $\mathrm{p}$ & $\begin{array}{c}\text { DSI maximale } \\
\text { sans effet clinique } \\
\text { observé }\end{array}$ \\
\hline \multirow{2}{*}{ Cétirizine (n = 166) } & $\mathrm{PSS}=0(\mathrm{n}=155)$ & $1,8 \pm 2,0$ & \multirow{2}{*}{0,00005} & \multirow{2}{*}{$9,4 \mathrm{mg} / \mathrm{kg}$} \\
\hline & $\mathrm{PSS}=1(\mathrm{n}=11)$ & $3,2 \pm 1,3$ & & \\
\hline \multirow{2}{*}{ Lévocétirizine $(\mathrm{n}=50)$} & $\mathrm{PSS}=0(\mathrm{n}=47)$ & $0,7 \pm 0,75$ & \multirow{2}{*}{0,015} & \multirow{2}{*}{$3,5 \mathrm{mg} / \mathrm{kg}$} \\
\hline & $\mathrm{PSS}=1(\mathrm{n}=3)$ & $2,1 \pm 1,2$ & & \\
\hline Féxofenadine $(\mathrm{n}=8)$ & PSS $=0(n=8)$ & $15,5 \pm 12,8$ & - & $38,5 \mathrm{mg} / \mathrm{kg}$ \\
\hline \multirow{2}{*}{ Loratadine $(\mathrm{n}=98)$} & $\mathrm{PSS}=0(\mathrm{n}=93)$ & $1,8 \pm 2,4$ & \multirow{2}{*}{0,06} & \multirow{2}{*}{$10 \mathrm{mg} / \mathrm{kg}$} \\
\hline & $\mathrm{PSS}=1(\mathrm{n}=5)$ & $2,4 \pm 1,2$ & & \\
\hline \multirow{2}{*}{ Desloratadine $(n=191)$} & PSS $=0(n=187)$ & $0,7 \pm 0,7$ & \multirow{2}{*}{0,003} & \multirow{2}{*}{$4,5 \mathrm{mg} / \mathrm{kg}$} \\
\hline & PSS $=1(n=4)$ & $2,1 \pm 0,8$ & & \\
\hline Mizolastine $(\mathrm{n}=10)$ & $\mathrm{PSS}=0(\mathrm{n}=10)$ & $0,8 \pm 0,4$ & - & $2 \mathrm{mg} / \mathrm{kg}$ \\
\hline \multirow{2}{*}{ Ébastine ( $n=46)$} & $\mathrm{PSS}=0(\mathrm{n}=42)$ & $1,6 \pm 1,3$ & \multirow{2}{*}{0,6} & \multirow{2}{*}{$5 \mathrm{mg} / \mathrm{kg}$} \\
\hline & PSS $=1(n=4)$ & $2,8 \pm 2,7$ & & \\
\hline
\end{tabular}

L'évolution était renseignée dans 461 dossiers d'exposition avec une guérison dans tous les cas. Tous les enfants avec PSS = 1 ont été suivis et ont évolué vers la guérison.

Conclusion: Pour la cétirizine, la lévocétirizine, la loratadine, la desloratadine et l'ébastine, une simple surveillance à domicile peut être recommandée pour les expositions accidentelles inférieures ou égales aux DSI maximales sans effet clinique observé, puisqu'à ces doses seuls des symptômes mineurs sont attendus. Un suivi évolutif par le CAPTV peut être préconisé à partir des doses moyennes avec effet (PSS $=1$ ). Pour la féxofénadine et la mizolastine, aucune conclusion n'est possible en raison du faible effectif de patients.

\section{C24. Pharmacobézoards et intoxications médicamenteuses volontaires}

G Durand, JC Gallart, F Pelissier, N Franchitto

Centre antipoison et de toxicovigilance de Toulouse Midi-Pyrénées et Limousin, Pôle de Médecine d'Urgences, CHU Toulouse-Purpan

Objectif : Dans la prise en charge des intoxications médicamenteuses volontaires, l'évolution doit être surveillée car elle se complique parfois d'aggravations retardées en relation avec la présence de pharmacobézoards, ou concrétions digestives médicamenteuses. Cette situation est propice à un relargage prolongé de toxiques et peut être objectivée par des dosages toxicologiques itératifs et des examens complémentaires.

Méthodes : Nous présentons le cas d'une patiente de 41 ans qui a absorbé de façon volontaire une quantité importante et non connue de clomipramine LP (Anafranil ${ }^{\circledR} 75$ LP).
Résultats : Une patiente dépressive de 41 ans, traitée par Anafranil ${ }^{\circledast} 75$ LP est découverte inconsciente à son domicile par ses parents. L'examen clinique initial par le médecin du SMUR retrouve un syndrome anticholinergique et sur l'ECG un effet stabilisant de membrane. La prise en charge immédiate consiste en une protection des voies aériennes supérieures par intubation orotrachéale et d'une réhydratation hydroélectrolytique, complétées à l'admission en réanimation d'une décontamination digestive par doses répétées de charbon activé. Les dosages toxicologiques sanguins initiaux retrouvent une concentration de clomipramine à $1489 \mu \mathrm{g} / \mathrm{L}$ (zone thérapeutique: $40-89 \mu \mathrm{g} / \mathrm{L}$ ) et la radiographie thoracique permet de visualiser la présence d'un conglomérat de comprimés dans la partie supérieure de l'estomac. L'état est stationnaire et les taux de clomipraminémies quotidiennes restent élevés, jusqu'à $1299 \mu \mathrm{g} / \mathrm{L}$ au $5^{\text {e }}$ jour. Évoquant l'existence d'un pharmacobézoard, une radiographie de l'abdomen sans préparation est alors réalisée et retrouve le conglomérat digestif identifié à l'entrée. Deux fibroscopies permettent alors le retrait de 35 puis de 50 comprimés. Seule une dizaine de comprimés ne peut être retirée et ce geste endoscopique visualise une gastrite hémorragique sans signe de gravité. Après le retrait des comprimés par fibroscopie, l'état clinique s'améliore en parallèle de la décroissance des taux de clomipramine qui atteignent la zone thérapeutique au dixième jour.

Conclusion : Dans la littérature, plusieurs facteurs favorisant la formation de pharmacobézoards lors d'intoxications aiguës ont été identifiés comme l'ingestion de formes à Libération Prolongée, un nombre important de comprimés ingérés ou le ralentissement du transit. Les risques liés à la présence d'un pharmacobézoard sont mécaniques par occlusion et toxicologiques par des relargages secondaires et imprévisibles de la substance active. Paradoxalement, un effet protecteur sur le plan toxicologique a également été décrit. La prise en charge thérapeutique n'est pas consensuelle et l'administration de doses répétées de charbon activé, la réalisation d'irrigation intestinale, d'endoscopie et la l'ablation chirurgicale ont été proposées. L'endoscopie a été plus souvent utilisée car elle permettait le diagnostic et l'évacuation du pharmacobézoard, sans avoir prouvé sa supériorité par rapport aux autres techniques. Le diagnostic de pharmacobézoard est donc important et repose sur des arguments anamnestiques, sur une évolution clinique défavorable et sur des dosages toxicologiques. En raison des risques d'aggravation associés sa présence justifie une admission en service de réanimation.

\section{C25. Risque d'hypoglycémie sévère lors de surdosage en Venlafaxine : à propos de 3 observations}

\section{$\underline{\text { C Bruneau }}^{1}$, D Perroux ${ }^{1}$, A Turcant ${ }^{2}$, B Lelievre ${ }^{2}$, S Ferec ${ }^{2}$, P Harry ${ }^{1}$}

${ }^{1}$ Centre Anti-Poison-Toxicovigilance, $\mathrm{CHU}$ d'Angers ; ${ }^{2}$ Laboratoire de Pharmaco-Toxicologie, $\mathrm{CHU}$ d'Angers

Objectif : Alerter sur les risques d'hypoglycémies sévères résistantes au glucose hypertonique lors des intoxications massives à la venlafaxine. En effet, cet antidépresseur bicyclique inhibe la recapture neuronale de la sérotonine et de la noradrénaline. L'effet hypoglycémiant de la sérotonine qui augmente la sensibilité à l'insuline est contrecarré par l'effet l'hyperglycémiant de la noradrénaline.

Méthodes : Tous les cas d'intoxication à la venlafaxine répertoriés au CAP du CHU d'Angers ont été recherchés. Seuls les cas où un dosage de venlafaxine a été réalisé par CLHP-UV/BD et où le patient a présenté des hypoglycémies ont été sélectionnés.

Résultats: Le CAP a recensé 3 intoxications à la venlafaxine où l'hypoglycémie persistait malgré les perfusions de glucose. Dans les 3 cas, 2 à 3 injections d'octréotide toutes les 8 à 12 heures ont permis la normalisation de la glycémie en 24 heures, réduit le débit des perfusions de glucose et supprimé les injections de glucose hypertonique. 


\begin{tabular}{|c|c|c|c|}
\hline & Cas $1: F, 39$ ans (1) & Cas $2: F, 44$ ans & Cas $3: F, 43$ ans \\
\hline $\begin{array}{l}\text { Doses } \\
\text { supposées } \\
\text { ingérées }\end{array}$ & $\begin{array}{l}\text { Venlafaxine } 7,5 \mathrm{~g} \text {, } \\
\text { alprazolam } 45 \mathrm{mg}\end{array}$ & $\begin{array}{l}\text { Venlafaxine } 10,5 \mathrm{~g}, \\
\text { loxapine } 1 \mathrm{~g}, \\
\text { quétiapine } 36 \mathrm{~g}, \\
\text { zopiclone } 315 \mathrm{mg}\end{array}$ & $\begin{array}{l}\text { Venlafaxine } 15,75 \mathrm{~g}, \\
\text { clorazépate } 450 \mathrm{mg}, \\
\text { zopiclone } 420 \mathrm{mg}, \\
\text { prednisolone } 800 \mathrm{mg}, \\
\text { amoxicilline } 12 \mathrm{~g}, \\
\text { métopimazine } 105 \mathrm{mg}, \\
\text { lévofloxacine } 1 \mathrm{~g}\end{array}$ \\
\hline Clinique & $\begin{array}{l}\text { Syndrome } \\
\text { sérotoninergique, } \\
\text { GS = 13/15. }\end{array}$ & $\begin{array}{l}\text { GS = 3/15, } \\
\text { hypotension, } \\
\text { convulsions }\end{array}$ & $\begin{array}{l}\text { GS = 10/15, } \\
\text { hypotension, } \\
\text { hypoventilation, } \\
\text { rhabdomyolyse }\end{array}$ \\
\hline Biologie & $\begin{array}{l}\text { Hypoglycémie récidivante } \\
(2,5 \mathrm{mmol} / \mathrm{L}), \\
\text { insuline }=83 \mu \mathrm{mol} / \mathrm{L}(\mathrm{N}), \\
\text { peptide } \mathrm{C}=1,9 \mathrm{nmol} / \mathrm{L}(\uparrow)\end{array}$ & \begin{tabular}{|l} 
Hypoglycémie \\
récidivante \\
$(3,9 \mathrm{mmol} / \mathrm{L})$
\end{tabular} & $\begin{array}{l}\text { Hypoglycémie récidivante } \\
(3,7 \mathrm{mmol} / \mathrm{L}), \\
\text { insuline }=9,6 \mu \mathrm{U} / \mathrm{mL}(\mathrm{N}) \text {, } \\
\text { peptide } \mathrm{C}=3,3 \mu \mathrm{g} / \mathrm{L}(\mathrm{N})\end{array}$ \\
\hline \begin{tabular}{|l}
$\begin{array}{l}\text { Analyse } \\
\text { toxicologique } \\
\text { (plasma) }\end{array}$ \\
\end{tabular} & $\begin{array}{l}\text { Venlafaxine* }=12 \mathrm{mg} / \mathrm{L} \\
\text { Sulfamide hypoglyc. : } 0\end{array}$ & Venlafaxine $*=9 \mathrm{mg} / \mathrm{L}$ & Venlafaxine ${ }^{*}=16 \mathrm{mg} / \mathrm{L}$ \\
\hline Traitement & $\begin{array}{l}\text { Glucosé } 10 \% \text { et } 30 \% \text {, } \\
\text { octréotide } 50 \mu \mathrm{g} \text { SC x2 }\end{array}$ & 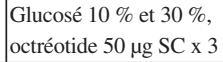 & $\begin{array}{l}\text { Glucosé } 10 \% \text { et } 30 \% \text {, } \\
\text { octréotide } 50 \mu \mathrm{g} \mathrm{SC} \times 2\end{array}$ \\
\hline
\end{tabular}

Conclusion : La venlafaxine induit un syndrome sérotoninergique associé à des concentrations d'insuline et de peptide Célevées ou normales en dépit de l'hypoglycémie. L'octréotide est un analogue de la somatostatine qui inhibe les sécrétions pancréatiques exocrines et endocrines. L'octréotide normalise la glycémie et diminue les besoins en glucose. Lors des intoxications massives à la venlafaxine, nous préconisons une surveillance étroite de la glycémie, l'administration d'octréotide en cas d'hypoglycémies récidivantes sous perfusion de glucose.

\section{$\overline{\text { C26. Deux cas d'intoxication aiguë par des carburants pour }}$ modèles réduits constitués de méthanol et de nitrométhane}

\section{Blanc, A Villa, S Maisant, D Chataigner, R Garnier}

Centre antipoison de Paris, Hôpital Fernand Widal, Paris

Introduction : Nous rapportons deux cas d'ingestion volontaire d'un carburant pour modèles réduits constitué d'un mélange de méthanol et de nitrométhane.

Cas cliniques : $\operatorname{Cas~}^{\circ} 1$ : Une femme de 46 ans dépressive, aurait ingéré volontairement, $48 \mathrm{~h}$ avant son admission, un verre de Rocket Fuel ${ }^{\circ}$ (70$99 \%$ méthanol et 1-30\% nitrométhane). À l'arrivée aux urgences, l'examen clinique était normal. La patiente se plaignait de céphalées, d'un flou visuel, de nausées, de brûlures gastriques et de diarrhée. Le bilan biologique montrait une créatininémie (dosé par la méthode de Jaffe) à $668 \mu \mathrm{mol} / \mathrm{L}$, une azotémie normale $(6,0 \mathrm{mmol} / \mathrm{L})$. Le reste du bilan biologique était normal. Quelques heures plus tard, un contrôle de la créatininémie par dosage enzymatique, ne montrait plus d'anomalie $(60,3 \mu \mathrm{mol} / \mathrm{L})$. La patiente est sortie contre avis médical le soir même. Devant la persistance de la dysphagie, elle a été hospitalisée à J15. Une oesogastroduodénoscopie ne montrait qu'une gastrite stade 1. Un bilan ophtalmologique systématique a mis en évidence une neuropathie optique : fond d'œil normal mais baisse de l'acuité visuelle (2/10 œil gauche, 4/10 œil droit), dyschromatopsie et scotome central bilatéral. Cas $\mathbf{n}^{\circ} 2$ : Un homme de 37 ans dépressif et éthylique chronique, est hospitalisé suspicion d'intoxication volontaire par le bromazépam et l'éthanol (J1). L'examen clinique montre un patient somnolent, une mydriase bilatérale, un nystagmus, une déviation de la tête et du regard à droite, des mouvements tonico-cloniques des membres supérieurs. Le bilan biologique met en évidence une acidose métabolique et un trou anionique élevé à 36,2 mais aussi une élévation importante de la créatininémie (dosage par méthode de Jaffé) à $4452 \mu \mathrm{mol} / \mathrm{L}$, alors que l'azotémie était normale $3,51 \mathrm{mmol} / \mathrm{L}$. Le scanner cérébral, la radio de thorax et l'ECG réalisés sont normaux. Le bilan toxicologique par immunochimie révèle la présence de benzodiazépines dans le sang; l'éthanol, le paracétamol, les barbituriques, et les antidépresseurs tricycliques sont indétectables. Le screening toxicologique urinaire par immunochimie ne révéle aucune autre substance. Le patient est transféré, à $\mathrm{H} 5$, en réanimation pour suspicion d'intoxication par le méthanol. À son arrivée, le score de Glasgow est de $12 / 15$, la pression artérielle systolique de $100 \mathrm{mmHg}$, la fréquence cardiaque de 90 battements/minute. La méthanolémie est de $1,87 \mathrm{~g} / \mathrm{L}$. Un traitement par 4-méthylpyrazole est initié à $\mathrm{H} 20$ et poursuivi jusqu'à $\mathrm{H} 51$; de H20 à H48 il est associé à une hémodialyse maintenue; de double traitement permet une correction rapide de l'acidose. À son réveil, à J2, le patient déclare avoir ingéré volontairement du carburant Modelbouw Brandstoff ${ }^{\circ}$ (> 50\% méthanol et 10-30\% nitrométhane) et se plaint d'une baisse de l'acuité visuelle. Les jours suivants les troubles visuels s'aggravent, cependant que la créatininémie (toujours mesurée par la méthode de Jaffé) diminue progressivement (jusqu'à $\mathrm{J} 7: 123 \mu \mathrm{mol} / \mathrm{L}$ ) ; pendant toute cette période l'azotémie reste normale. À J7, l'examen ophtalmologique montre une atrophie optique bilatérale avec disparition des contenus papillaires et une semi-mydriase aréflexique.

Conclusion: Les carburants pour modèles réduits (avions, voitures, bateaux...) sont généralement composés de nitrométhane et de méthanol. Ces produits accessibles au public sont potentiellement responsables d'intoxications graves par le méthanol. Le nitrométhane ne semble pas constituer un facteur aggravant, mais il est responsable de fausses élévations de la créatinine sérique lorsque celle-ci est mesurée avec la méthode de Jaffe, en raison d'interférences colorimétriques.

\section{$\overline{\text { C27. Éthyl glucuronide dans les urines : étude de stabilité et }}$ données en toxicologie clinique}

M Le Merdy, X Decleves, L Labat

Plateforme Biologie du Médicament, Groupe Cochin, AP-HP, Paris

Objectif : Dans le cadre d'une étude randomisée en milieu ambulatoire ayant pour objectif de montrer l'efficacité à un an du bacloféne sur la consommation d'alcool, un bilan biologique comprenant g-GT, CDT, transaminases et numération formule sanguine est réalisé permettant une évaluation indirecte de la poursuite de l'intoxication alcoolique. Afin de mieux évaluer cette consommation alcoolique, il est proposé lors de la visite de sortie de l'étude de réaliser le dosage de l'éthyl glucuronide (EtG) dans les urines. Les inclusions de cette étude multicentrique étant réalisées dans de nombreuses communes de France, le problème de conservation des prélèvements urinaires avant acheminement apparaît comme une limite au dosage de ce marqueur. Nous avons donc envisagé une étude de stabilité complète sur trois semaines de différents prélèvements urinaires pour apprécier la faisabilité de ce dosage dans les conditions de cette étude.

Méthodes : Six urines de témoins ( 2 femmes, 4 hommes) considérés comme buveurs sociaux ou alcooliques chroniques sont recueillies le matin. Seuls les buveurs sociaux ont estimé leur consommation de la veille entre $10 \mathrm{~g}$ et $100 \mathrm{~g}$ d'alcool. Les dosages d'EtG (DRI, Microgenics, cut off $0,5 \mathrm{mg} / \mathrm{L}$ ), d'éthanol et de créatinine urinaires ainsi qu'une analyse cytobactériologique sont réalisés au moment du recueil. Le dosage d'EtG est testé sur des tubes contenant de l'acide borique (V-Monovette urine, Sarstedt) et sur des flacons type ECBU sans conservateur. On réalise l'étude de stabilité pour chaque urine pendant 3 semaines dans des conditions différentes de température $\left(4{ }^{\circ} \mathrm{C}\right.$, température ambiante), d'oxydation et de lumière. En parallèle une étude de stabilité à $-20^{\circ} \mathrm{C}$ est réalisée pendant 3 mois.

Résultats : Les prélèvements urinaires réalisés sur des tubes contenant de l'acide borique ont tous donné des résultats faussement négatifs. Les concentrations mesurées dans les flacons type ECBU varient de 0,4 à $17,9 \mathrm{mg} / \mathrm{g}$. créat. (entre $\mathrm{H} 12$ et H18) pour la catégorie des buveurs sociaux $(\mathrm{n}=4)$ et de 88,3 à $125,2 \mathrm{mg} / \mathrm{g}$. créat. pour les alcooliques chroniques $(\mathrm{n}=2)$. Un seul des prélèvements est positif à l'éthanol $(0,4 \mathrm{~g} / \mathrm{L})$. L'étude menée sur une période de trois semaines montre une excellente stabilité pour l'ensemble 
des conditions étudiées (CV entre 3,1\% et 13,5\%). La congélation à 1 mois est également stable pour ce marqueur $(\mathrm{CV}<13,9 \%)$. Pour le témoin LA02 (femme), une concentration proche du cut off, $0,6 \mathrm{mg} / \mathrm{L}$ soit $2,3 \mathrm{mg} / \mathrm{g}$. créat (H13) pour une consommation de $10 \mathrm{~g}$ et une concentration de $13,9 \mathrm{mg} / \mathrm{L}$ soit $17,9 \mathrm{mg} / \mathrm{g}$. créat. (H13) pour une consommation de $50 \mathrm{~g}$ sont mesurées. De même pour le témoin MA01 (homme), des concentrations de 1,2 mg/L soit $0,6 \mathrm{mg} / \mathrm{g}$.créat $(\mathrm{H} 15)$ pour $30 \mathrm{~g}$ consommés et $11,8 \mathrm{mg} / \mathrm{L}$ soit $7,4 \mathrm{mg} / \mathrm{g}$. créat. (H15) pour $75 \mathrm{~g}$ consommés sont mesurées.

Conclusion : Les résultats sur les tubes contenant de l'acide borique ajouté pour éviter la prolifération bactérienne pouvant fausser le dosage de l'EtG dans les urines, ne sont pas utilisables. Pour l'étude en ambulatoire, nos résultats montrent qu'il n'y a aucune précaution particulière à prendre en terme de conservation des échantillons et que des pots type ECBU peuvent être utilisés. Comme décrit dans la littérature, les valeurs mesurées en EtG pour les femmes sont toujours supérieures à celles des hommes à consommation équivalente. Pour LA02 et MA01, les différences de concentrations entre les deux situations peuvent être expliquées par une saturation des voies oxydatives principales de l'alcool (voie de l'ADH entre autres) à l'origine d'une surexpression de la voie de l'UDP-Glucuronosyltransferase (UGT) et d'une augmentation importante de l'EtG. On peut supposer que cette voie minoritaire non oxydative en situation de faible consommation devient plus importante dans les cas de consommations plus élevées. Une étude de cinétique d'élimination urinaire à doses variables est envisagée avec ces mêmes témoins.

\section{C28. Une interférence analytique peut en cacher une autre : exemple de l'urapidil, du dépistage de MDMA et de l'identification d'un stimulant de synthèse, le 2-MeOPP}

$\underline{\mathrm{N}}$ Allibe $^{1}$, X Fonrose ${ }^{2}$, M Bartoli ${ }^{2}$, M Barbieux ${ }^{3}$, H Eysseric ${ }^{1,2}$, F StankeLabesque $^{2}$

${ }^{1}$ Laboratoire de Médecine Légale, UJF Grenoble; ${ }^{2}$ UM Pharmacologietoxicologie, CHU Grenoble ; ${ }^{3}$ Pathologie Neuro Vasculaire, CHU Grenoble

Objectif : Un dépistage urinaire de MDMA a été réalisé dans le cadre d'une recherche systématique d'étiologie toxique lors d'un accident vasculaire cérébral (AVC) chez un patient âgé de 53 ans. Le résultat obtenu par dépistage immunochimique de MDMA était bien supérieur au seuil de positivité $(1098 \mu \mathrm{g} / \mathrm{L})$. Le patient niant formellement la consommation de toute substance illicite, nous avons mis en œuvre des analyses chromatographiques pour confirmer ce résultat ou identifier une éventuelle interférence analytique.

Méthodes : Le dépistage immunochimique de MDMA (IC/MDMA) dans les urines a été réalisé à l'aide du kit Syva ${ }^{\circledR}$ EMIT $^{\circledR}$ Plus Ecstasy utilisé sur un système Dimension Vista ${ }^{\circledR}$ avec un seuil de positivité fixé à $300 \mu \mathrm{g} / \mathrm{L}$. Les criblages chromatographiques ont été réalisés par une technique de CLBD (Shimadzu Prominence, colonne C18 nucleosil) et par une technique de CPG-SM (5975 Inert XL MSD, Agilent, colonne DB-5MS UI). Le dosage spécifique du MDMA et du MDA a été réalisé en CL-SM/SM (Shimadzu Prominence, colonne Atlantis, AB SCIEX 3200 QTRAP).

Résultats: Le dosage spécifique en CL-SM/SM n'a pas confirmé la présence de MDA et MDMA (limite de détection à $1 \mu \mathrm{g} / \mathrm{L}$ ). Le screening réalisé en CPG-SM a mis en évidence la présence de (1,2-méthoxyphényl)pipérazine (2-MeOPP), un stimulant de synthèse. Le criblage réalisé en CL-BD a permis de détecter et quantifier de l'urapidil, un vasodilatateur, à la concentration de $94 \mathrm{mg} / \mathrm{L}$, administré lors de la prise en charge médicale. Des solutions de 2-MeOPP et urapidil testées en IC/MDMA montrent une absence de signal avec le 2-MeOPP et un signal positif avec l'urapidil. L'analogie structurale entre ces 2 composés nous permet de supposer que le vasodilatateur, ou l'un de ses métabolites, est dégradé par nos conditions chromatographiques de CPG-SM et génère un pic de 2-MeOPP. Afin de confirmer cette interférence en IC/MDMA, nous avons testés différentes urines de patients traités par urapidil. Le signal obtenu était soit proche, soit supérieur au seuil de positivité confirmant l'hypothèse d'une interférence par l'urapidil et/ou ses métabolites.

Conclusion : Nous avons mis en évidence un double risque d'interférence de l'urapidil non seulement avec le kit Syva ${ }^{\circledR}$ EMIT $^{\circledR}$ Plus Ecstasy mais également avec l'identification de 2-MeOPP en CPG-SM. Ce travail montre qu'il peut être utile de confirmer la présence de composés à l'aide de plusieurs techniques complémentaires afin d'éviter le piège de faux positif.

\section{C29. Un toxique peut en cacher un autre}

C Tournoud ${ }^{1}$, A Charton ${ }^{2}$, V Cirimele ${ }^{3}, \underline{\text { F Flesch }}^{1}$

${ }^{1}$ Centre antipoison, Nouvel Hôpital Civil, Strasbourg; ${ }^{2}$ Réanimation Chirurgicale, Hôpital de Hautepierre, Strasbourg; ${ }^{3}$ Laboratoire Chemtox, Illkirch

Introduction : Certaines intoxications posent de réels problèmes diagnostiques. L'aide des toxicologues analystes peut alors s'avérer indispensable, y compris en urgence, pour adapter la prise en charge thérapeutique du patient.

Cas clinique : Un homme, âgé de 45 ans, est retrouvé sur la voie publique, en coma Glasgow 3, à côté de plusieurs boites vides de médicaments : Seresta $₫$, Nuctalon $®$, Valdoxan $®$, Valium $®$, Zopiclone, Zolpidem. Après intubation, le patient est transféré en réanimation. Le bilan d'admission

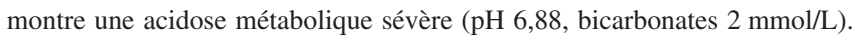
Un réveil partiel transitoire est obtenu après administration de flumazenil. L'évolution se traduit par la réapparition rapide du coma, l'installation d'une insuffisance rénale aiguë anurique et d'une hyperammoniémie à $154 \mu \mathrm{mol} / \mathrm{L}$ ainsi que l'aggravation de l'acidose ( $\mathrm{pH}$ 6,76). Les dosages d'aspirine, de valproate, de GHB et de méthanol réalisés à l'admission sont négatifs. Le dosage d'éthylène glycol n'est pas disponible. Le patient est stable sous vasopresseurs, avec correction de l'acidose sous hémodiafiltration continue (HDFC). Trente six heures après l'admission, le dialogue entre réanimateur et médecin du Centre antipoison permet de reprendre les différents éléments chronologiques de l'observation clinique, de confirmer l'existence initiale d'un trou anionique à $39 \mathrm{mmol} / \mathrm{L}$, d'un trou osmolaire à $55 \mathrm{mosm} / \mathrm{L}$ et d'évoquer une intoxication à l'éthylène glycol : l'indication d'un traitement par fomépizole est alors posée. La confirmation de l'intoxication est obtenue 24 heures plus tard grâce aux dosages réalisés sur les prélèvements initiaux qui ont pu être réacheminés vers le laboratoire réalisant le dosage (par GC/ MS après dérivation par BSTFA/TMCS). Les concentrations sanguine et urinaire d'éthylène glycol étaient respectivement de 2,51 g/L et 7,4 g/L (une intoxication est potentiellement sévère à partir d'une concentration sérique supérieure à $500 \mathrm{mg} / \mathrm{L}$ ). L'état du patient s'est progressivement amélioré sous HDFC et traitement par fomépizole. Le réveil a été lent et progressif avec néanmoins persistance de séquelles neurologiques. L'IRM cérébrale (à 1 mois) montrait des lésions nécrotiques et hémorragiques de la substance blanche sous corticale temporo-occipitale, pariétale et frontale bilatérales.

Discussion : Cette observation permet de souligner la nécessité d'une collaboration étroite et permanente entre le médecin du Centre antipoison et les réanimateurs, la nécessité de remettre en question à tout moment les hypothèses diagnostiques et celle de trouver un laboratoire susceptible de réaliser, en urgence, les dosages pouvant confirmer ou infirmer ces hypothèses. Dans le cas présent, l'ensemble de ces facteurs aurait probablement permis une mise en route plus précoce du traitement antidotique et, peut-être, une évolution séquellaire moins grave.

Conclusion : Le diagnostic d'une intoxication à l'éthylène glycol repose sur l'association d'un coma avec une acidose métabolique non lactique, un trou anionique et un trou osmolaire augmentés. Dans les cas d'intoxications sévères, une collaboration étroite entre les réanimateurs, les toxicologues cliniciens et les toxicologues analystes est essentielle. 


\section{C30. Évaluation de l'intérêt du criblage toxicologique demandé par les services de réanimation et d'urgences au CHU de Grenoble}

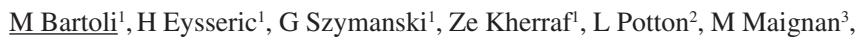
F Stanke-Labesque ${ }^{1}$

${ }^{1}$ UM Pharmacologie et Toxicologie, CHU, Grenoble; ${ }^{2} U M$ Réanimation Médicale CHU, Grenoble ; ${ }^{3} \mathrm{UM}$ Urgences Médicales, $\mathrm{CHU}$, Grenoble

Objectif : Suite à la question posée par le groupe de travail «toxicologie clinique » sur le bénéfice de la mise en œuvre de criblage toxicologique pour la prise en charge des patients dans les intoxications graves (Bartoli et al., Ann Biol Clin. 2012), nous avons réalisé une évaluation prospective de la justification et de l'utilité de ces demandes, prescrites par les services de réanimation et des

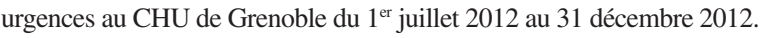

Méthodes : Le criblage toxicologique a concerné le plasma et/ou les urines. Il a débuté systématiquement par une analyse en CL-BD (Prominence ${ }^{\circledR}$ Shimadzu) complétée suivant la symptomatologie et les toxiques suspectés, par CPG-SM (6890N/5975 Agilent) et/ou CL-SM/SM (Prominence ${ }^{\circledR}$ Shimadzu, 3200 QTRAP ${ }^{\circledR}$ AB Sciex). Chaque prescription de criblage a fait l'objet d'un dialogue clinico-biologique afin d'en préciser son contexte (intoxication médicamenteuse grave, absence d'orientation diagnostique, contexte médicolégal). L'utilité du criblage a été évaluée selon les critères suivants : aide à l'établissement du diagnostic (confirmation d'intoxication suspectée, exclusion, mise en évidence d'une étiologie toxique, identification de toxiques autres que ceux suspectés) et/ou modification de la prise en charge du patient. Résultats : Durant cette période, 82 criblages ont été demandés par les médecins cliniciens, 19 par les services des urgences et 63 par les services de réanimation. 19 demandes (23\%) n'ont pas été réalisées après accord avec le clinicien du fait de l'absence de justification. 63 demandes ont donc fait l'objet d'analyses, $51 \%$ pour absence d'orientation diagnostique, $43 \%$ pour contexte d'intoxication médicamenteuse et $6 \%$ pour contexte médico-légal. L'évaluation de l'utilité des analyses figure dans le tableau :

\begin{tabular}{|l|c|c|c|c|c|c|}
\hline $\begin{array}{l}\text { Utilité } \\
\text { Contexte }\end{array}$ & $\begin{array}{c}\text { Confirmation } \\
\text { intoxication }\end{array}$ & $\begin{array}{c}\text { Exclusion } \\
\text { intoxication }\end{array}$ & $\begin{array}{c}\text { Découverte } \\
\text { étiologie } \\
\text { toxique }\end{array}$ & $\begin{array}{c}\text { Autres } \\
\text { toxiques } \\
\text { retrouvés }\end{array}$ & $\begin{array}{c}\text { Aucune } \\
\text { utilité }\end{array}$ & $\begin{array}{c}\text { Modification } \\
\text { de la prise } \\
\text { en charge }\end{array}$ \\
\hline $\begin{array}{l}\text { Absence } \\
\text { d'orientation } \mathrm{n}=32\end{array}$ & 0 & $23(72 \%)$ & $7(22 \%)$ & 0 & $2(6 \%)$ & $3(9 \%)$ \\
\hline $\begin{array}{l}\text { Suspicion } \\
\text { d'intoxication } \\
\mathrm{n}=27\end{array}$ & $20(74 \%)$ & $1(4 \%)$ & 0 & $6(22 \%)$ & 0 & $3(11 \%)$ \\
\hline Médico-légal $\mathrm{n}=4$ & 0 & $4(100 \%)$ & 0 & 0 & 0 & 0 \\
\hline
\end{tabular}

Conclusion : Après un premier filtre effectué par le biologiste et en accord avec le clinicien, seulement $3 \%$ des criblages n'ont eu aucune utilité. Le diagnostic d'exclusion est largement majoritaire lors de l'absence d'orientation diagnostique et dans le cadre médico-légal. En cas de suspicion d'intoxication, le diagnostic de confirmation prime. Le criblage a permis également de découvrir une étiologie toxique dans $22 \%$ des contextes d'absence d'orientation et de dévoiler d'autres toxiques dans $22 \%$ des cas de suspicion d'intoxication. Au total la prise en charge est modifiée dans $10 \%$ des cas. Ces résultats confirment l'utilité du criblage toxicologique lorsque son indication est bien posée.

\section{C31. Intoxication mortelle à la colchicine chez une patiente de 22 ans : articulation entre médecine pré-hospitalière, CAPTV et réanimation}

\section{JC Gallart, F Pelissier, N Franchitto}

Centre antipoison et de toxicovigilance de Toulouse Midi-Pyrénées et Limousin, Pôle de Médecine d'Urgence, CHU Toulouse-Purpan

Objectif: Les intoxications volontaires par la colchicine restent peu fréquentes et la gravité potentielle nécessite une parfaite articulation des différents acteurs pré-hospitaliers, hospitaliers et des CAPTV.
Méthodes : Description de la prise en charge d'une patiente intoxiquée par la Colchicine.

Cas clinique : Une jeune femme de 22 ans a contacté le SAMU 30 minutes après avoir ingéré 40 milligrammes de colchicine $(0,7 \mathrm{mg} / \mathrm{kg})$ et deux comprimés de Noctran ${ }^{\circledR} 10 \mathrm{mg}$ dans un but suicidaire. À l'appel, elle est asymptomatique et à l'arrivée de l'équipe médicale, la pression artérielle est mesurée à 130/84 mmHg, symétrique, la fréquence cardiaque à 85 battements par minute et l'ECG est normal. La patiente est transportée jusqu'au Centre Hospitalier Universitaire, situé à 40 minutes du domicile. À l'admission aux soins intensifs, aucun signe clinique de défaillance viscérale n'est présent, et le bilan biologique est normal. La colchicinémie est mesurée à $10,9 \mu \mathrm{g} / \mathrm{L}$ et les autres toxiques sont négatifs. Devant la dose supposée ingérée de colchicine, un lavage gastrique et l'administration de charbon activé sont réalisés. Sept heures après l'ingestion, l'état hémodynamique de la patiente se dégrade brutalement, avec une cytolyse hépatique et l'ECG retrouve des troubles de la repolarisation. Une écho-cardiographie transthoracique permet de mesurer une fraction d'éjection ventriculaire gauche à $15 \%$. Un traitement symptomatique de la défaillance cardio-circulatoire associe alors des catécholamines (Dobutamine), une épuration rénale continue et secondairement une assistance circulatoire extra-corporelle ou ECMO. Deux jours après l'admission, la concentration de Colchicine reste élevée à $7,9 \mu \mathrm{g} / \mathrm{L}$. Malgré ces traitements, la patiente décède 3 jours après l'ingestion. Conclusion : La colchicine est un alcaloïde naturel utilisé comme agent antimitotique et anti-inflammatoire dans le traitement de la crise de goutte, de la chondrocalcinose articulaire du lupus, de la sclérodermie ou de la fièvre Méditerranéenne familiale. Elle a un index thérapeutique étroit et une toxicité intrinsèque importante, qui participe à la sévérité du pronostic. Sur le plan thérapeutique, la prise en charge fait appel à une décontamination digestive précoce, des traitements symptomatiques et de suppléance. En France, le constat d'une augmentation récente des cas graves et des décès a relancé la recherche sur l'immunothérapie par anticorps spécifique anti-colchicine. Au regard des résultats encourageants déjà obtenus en expérimentation animale, cette alternative thérapeutique pourrait être efficace dans les intoxications humaines les plus sévères. Dans le cas présenté ici, le recours précoce à l'utilisation de charbon activé en pré-hospitalier a été évoqué sans garantie qu'il aurait modifié l'évolution de cette intoxication à la mortalité proche de $50 \%$ (en raison d'un dose supposée ingérée comprise entre 0,5 et $0,8 \mathrm{mg} / \mathrm{kg}$ ). On peut aussi penser que le recours aux anticorps spécifiques aurait pu modifier cette évolution défavorable.

\section{$\overline{\text { C32. Baclofène et encéphalopathie : à propos d'un surdosage }}$ chez un patient insuffisant rénal}

AS Lemaire-Hurtel $^{1}$, A Cheyroux ${ }^{2}$, C Presne ${ }^{3}$, A Knapp ${ }^{4}, \mathrm{~K}^{2}$ Masmoudi ${ }^{2}$, $\mathrm{V} \mathrm{Gras}^{2}$, JC Alvarez ${ }^{4}, \mathrm{M}^{\text {Andrejak }}{ }^{1,2}$

${ }^{1}$ Laboratoire de Pharmacologie et Toxicologie-CHU Amiens; ${ }^{2}$ Pharmacovigilance-CHU Amiens ; ${ }^{3}$ Service de Néphrologie-CHU Amiens ; ${ }^{4}$ Laboratoire de Pharmacologie et Toxicologie-CHU Garches

Objectifs : Le baclofène (Liorésal ${ }^{\circledR}$ ) est un myorelaxant d'action centrale, analogue du GABA, prescrit en France depuis près de 40 ans dans le traitement de la spasticité centrale cérébrale ou médullaire (hémiplégie ou paraplégie spastique). Nous rapportons le cas d'un patient de 60 ans, insuffisant rénal chronique (clairance à la créatinine à $20 \mathrm{~mL} / \mathrm{min}$ ), traité quotidiennement par $15 \mathrm{mg}$ de baclofène pour la prise en charge d'une hémiplégie droite spastique post AVC hémorragique secondaire à une HTA sévère non suivie depuis 2004. Suite à la découverte d'une volumineuse tumeur rénale, ce patient a été récemment admis en chirurgie pour une néphrectomie élargie droite. Son traitement comportait de l'aténolol $1000 \mathrm{mg} \times 2 / \mathrm{j}$, amlodipine $10 \mathrm{mg} / \mathrm{j}$, rilmenidine $1 \mathrm{mg} \times 2 / \mathrm{j}$, zocor $40 \mathrm{mg} / \mathrm{j}$, coversyl $10 \mathrm{mg} / \mathrm{j}$, novonorm $1 \mathrm{mg} / \mathrm{j}$, kardégic $75 \mathrm{mg} / \mathrm{j}$, lioresal $1 / 2 \times 3 / \mathrm{j}$, furosémide $10 \mathrm{mg} / \mathrm{j}$ et Mimpara $30 \mathrm{mg} / \mathrm{j}$. 
L'hospitalisation s'est compliquée d'un choc hémorragique et d'une encéphalopathie 7 jours après l'intervention.

Méthodes : Un double screening en CG/SM (Shimadzu QP2010) et CLHP/ BD (Waters, Alliance) est demandé sur 2 échantillons sanguins prélevés avant et après dialyse du patient. La concentration de baclofène est mesurée par une méthode validée utilisant la chromatographie liquide couplée à un spectromètre de masse à trappe d'ions, après double extraction. La séparation des analytes est effectuée en phase inverse sur une colonne C18 de 5- $\mu \mathrm{m}$, l'élution isocratique est assurée par un mélange de tampon formiate et d'acétonitrile à $200 \mu \mathrm{L} / \mathrm{min}$. La détection se fait en modes full-scan MS et full-scan MS/MS en sélectionnant les rapports m/z 214.0 et $\mathrm{m} / \mathrm{z} 218.0$ respectivement pour le baclofène et baclofène-D4, l'étalon interne retenu. La méthode est linéaire de 20 à $2000 \mathrm{ng} / \mathrm{mL}$. La répétabilité et la fidélité intermédiaire ont été mesurées par l'analyse de 18 contrôles de qualité à 3 niveaux de concentrations différents : $70 \mathrm{ng} / \mathrm{mL}, 600 \mathrm{ng} / \mathrm{mL}$ et $1200 \mathrm{ng} /$ $\mathrm{mL}$. Pour ces 3 niveaux, les tests de répétabilité et de fidélité intermédiaire correspondaient aux critères attendus $(\mathrm{CV}<15 \%)$. Les LOD et LOQ sont respectivement de 10 et $20 \mathrm{ng} / \mathrm{mL}$.

Résultats : Sept jours après l'intervention, le patient présente un épisode de confusion avec hypotension. Sa fonction rénale s'est nettement dégradée (insuffisance rénale aiguë avec clairance à la créatinine à $10 \mathrm{~mL} / \mathrm{min}$ ). Le scanner cérébral ne retrouve pas de signe d'AVC. Un surdosage médicamenteux est évoqué. Le screening toxicologique montre la présence de baclofène. Les concentrations sériques de baclofène sont mesurées à $2100 \mathrm{ng} / \mathrm{mL}$ sur le prélèvement effectué avant la dialyse, et à $1150 \mathrm{ng} / \mathrm{mL}$ sur le prélèvement post dialyse, les concentrations habituellement efficaces étant comprises entre 80 et $160 \mathrm{ng} / \mathrm{mL}$ et celles toxiques entre 100 et $3500 \mathrm{ng} / \mathrm{mL}$. La différence observée entre les 2 prélèvements signifie que la dialyse a permis l'élimination de la moitié du médicament.

Conclusion: L'encéphalopathie est très probablement la conséquence d'un surdosage en baclofène, conséquence d'une activation excessive des récepteurs GABAergiques. La régression des symptômes après une séance d'hémodialyse, corrélée à une nette diminution des concentrations sériques, va dans le sens de cette hypothèse. En effet, le baclofène est majoritairement éliminé par filtration glomérulaire. Chez les sujets à fonction rénale normale, $80 \%$ de la dose administrée est retrouvée sous forme inchangée dans les urines 24 heures après l'administration. Ce médicament s'accumule chez les patients dont la fonction rénale est altérée. Il existe dans la littérature plusieurs observations d'encéphalopathies induites par le baclofène. Elles concernent des patients insuffisants rénaux dialysés traités à des posologies normales de médicament. Cette observation montre l'intérêt de la dialyse qui a permis d'épurer ce médicament, réduisant cliniquement l'atteinte neurologique. Il est donc nécessaire de rappeler la nécessité de baisser les posologies chez les patients présentant une fonction rénale altérée, d'autant plus qu'il n'existe pas d'antidote au baclofène.

\section{C33. Administration criminelle de mifépristone (RU486) et de misoprostol : à propos de $\mathbf{2}$ cas}

M Cheze, G Hoizey, M Deveaux, A Muckensturm, E Medrano, F Billault, G Pepin

\section{Laboratoire Toxlab, Paris}

Introduction : L'administration criminelle de médicaments abortifs comme la mifépristone (Mifégine ${ }^{\circledR}$, RU486) et le misoprostol $\left(\right.$ Gymiso $^{\circledR}$ ou Cytotec $^{\circledR}$, MIS) est très rare. Les auteurs décrivent 2 cas où le conjoint a fait prendre à la mère, à son insu et dans le but de provoquer un avortement, du RU486 (Cas\#1) et du MIS (Cas\#2).

Description des cas : $\underline{\text { Cas\#1 }}$ : Au décours d'une hospitalisation d'urgence pour fausse-couche sans cause médicale apparente, le laboratoire est requis en urgence pour rechercher les médicaments ayant un fort potentiel abortif ; une recherche large de médicaments et de stupéfiants a également été effectuée Les échantillons analysés sont : sang de la mère $(\mathrm{T}+21 \mathrm{~h})$; foie, poumon et reins du fœtus. $\underline{\text { Cas\#2 }}$ : Dans un scénario identique, les échantillons analysés sont : sérum de la mère $(T+3 h)$, urine de la mère $(T+72 h)$, eau de boisson ayant pu contenir le médicament, placenta.

Méthodes : La recherche de RU486 (m/z 430.3 $\rightarrow 372.2$, 288.2, 236.2, 134.2), de misoprostol $(\mathrm{m} / \mathrm{z} 363.3 \rightarrow 333.2,315.2,233.2,217.2)$ et de misoprostol acide $(\mathrm{m} / \mathrm{z} 367.1 \rightarrow 249.0)$ était effectuée par ESI(+)-LC-MS/ MS en mode MRM sur les extraits de prélèvements biologiques et sur une dilution de l'eau de boisson. La séparation chromatographique était réalisée sur une colonne Uptisphère ODB C18 $5 \mu \mathrm{m}, 150 \times 2 \mathrm{~mm}$ thermostatée à $30{ }^{\circ} \mathrm{C}$. La phase mobile était délivrée en gradient de $85 \%$ tampon formiate $2 \mathrm{mM} \mathrm{pH} 3$ et $15 \%$ d'acétonitrile augmenté à $90 \%$ en 10 min puis rééquilibré aux conditions initiales en $5 \mathrm{~min}$. La durée de l'analyse est de $15 \mathrm{~min}$. La recherche large d'autres médicaments et de stupéfiants a été effectuée en CLHP-BD et en CPG-SM.

Résultats : Cas\#1 du RU486 a été retrouvée aux concentrations suivantes : sang de la mère $131,4 \mathrm{ng} / \mathrm{mL}$; fotus : foie $0,35 \mathrm{ng} / \mathrm{g}$, poumon $0,56 \mathrm{ng} / \mathrm{g}$, reins $0,83 \mathrm{ng} / \mathrm{g}$. Aucun autre médicament n'a été détecté, notamment pas de MIS. $\underline{\text { Cas\#2 }}$ : Le MIS n'a pas été détecté dans le sérum et l'urine de la mère, mais dans l'eau : $250 \mathrm{ng} / \mathrm{mL}$; il a été retrouvé du MIS acide (métabolite du MIS) dans le placenta. Ni le RU486 ni aucun autre médicament n'ont été détectés.

Discussion : Le RU486 est un stéroïde de synthèse à action antiprogestative, indiqué dans l'IVG en respectant le cadre réglementaire des dispositions de l'article R.2212-10 du CSP, ou après mort in-utero. La concentration retrouvée dans le sang de la mère (Cas\#1) est en accord avec le prélèvement tardif et la demi-vie $(20-85 \mathrm{~h})$ car la concentration sanguine maximale habituellement retrouvée 1,5 à $2 \mathrm{~h}$ après la prise de 25 à $600 \mathrm{mg}$ va de 750 à $1910 \mathrm{ng} / \mathrm{mL}$. Des recherches ultérieures dans les plats ayant pu contenir le RU486 dissimulé dans les aliments seront effectuées si l'enquête le nécessite. Pour le MIS (analogue de prostaglandine) dans le Cas\#2, seules les analyses de l'eau de boisson et du placenta ont permis de confirmer son administration. Sa demi-vie extrêmement courte (20-40 min) rendait illusoire toute recherche efficace dans le sang et l'urine de la mère. La quantité totale présente dans la bouteille a été estimée à $400 \mu \mathrm{g}$, qui correspondent à la posologie habituelle, mais un seul verre avait été consommé. Le conjoint s'était fait prescrire du Cytotec ${ }^{\circledR}$ pour l'administrer à sa femme. Le RU486 et le MIS sont normalement être administrés successivement.

Conclusion : Si l'enquête le nécessite dans certains cas de fausse-couche sans cause médicale avérée, il est donc tout à fait possible de mettre en évidence le RU486 et le MIS utilisés habituellement dans les IVG. Cette recherche est possible en urgence, notamment dans des prélèvements inhabituels comme le placenta et le fotus, étant donné la demi-vie très courte de ces médicaments.

\section{C34. Dermatite flagellaire au shiitake Lentinus edodes cru : une alerte des centres antipoison français}

D Boels ${ }^{1}$, A Landreau ${ }^{1}, \mathrm{R}_{\text {Garnier }}^{2}$, C Pulce ${ }^{3}$, L De Haro $^{4}$, A Caubet $^{5}$, P Harry ${ }^{1}$ ${ }^{I}$ Centre antipoison - toxicovigilance, $\mathrm{CHU}$ Angers; ${ }^{2}$ Centre antipoison - toxicovigilance, Hôpital Fernand Widal, Paris; ${ }^{3}$ Centre antipoison toxicovigilance, CHU Lyon; ${ }^{4}$ Centre antipoison - toxicovigilance, Hôpital Salvator, Marseille; Centre antipoison - toxicovigilance, ${ }^{5} \mathrm{CHU}$ Pontchaillou, Rennes

Objectifs : Les premières intoxications à l'origine d'une toxidermie au champignon Lentinus edodes appelé plus communément shiitake ont été décrites en 1974 au Japon. Cette problématique semble être de plus en plus fréquente en Europe et notamment en France bien qu'elle reste méconnue.

Matériels et méthodes : Il s'agit d'une étude rétrospective des cas d'exposition au shiitake saisis sur le système informatique (SICAP) des CAPTV entre janvier 2000 et février 2013. Les critères de tri dans le système d'exploitation Infocentre ont porté sur les libellés shiitake, lentinan, Lentinus edodes de la classe «agent». Un cas est défini par toute personne exposée à l'un de ces 
agents. Les demandes d'information et les dossiers pour lesquels il s'avère que les champignons en cause n'étaient pas des shiitake ont été exclus.

Résultats : Sur les 42 dossiers, 22 dossiers ont été exclus. Sur les 20 dossiers restants (30 exposés), 6 cas d'ingestion de shiitake ont été suivis de troubles digestifs mineurs (douleurs abdominales, nausées, vomissements, diarrhées) d'évolution spontanément favorable ne nécessitant pas d'hospitalisation sans que nous puissions préciser le mode de cuisson et les aliments associés et donc confirmer ou infirmer le rôle du shiitake. Dans 10 cas, les consommateurs de shiitake sont restés asymptomatiques.

Pour 14 personnes, la consommation de shiitake a été suivie d'une éruption cutanée et d'un prurit intense. Pour 11 personnes, le diagnostic de dermatite flagellaire au shiitake est très probable avec une chronologie des symptômes évocatrice. Cette toxidermie a été observée, dans 10 cas, suite à la consommation de shiitake crus ou peu cuits qu'ils soient frais ou réhydratés. Dans 2 cas, les shiitake ont été consommés pour un usage médicinal (en infusion pour l'un et en gélule de poudre pour l'autre).

L'éruption cutanée a débuté entre $12 \mathrm{~h}$ et 5 jours après l'ingestion des champignons avec une médiane à $24 \mathrm{~h}$. Cette éruption prurigineuse papuleuse caractéristique formant des lignes évoquant des « griffures » s'est généralisée au tronc puis aux extrémités en $24 \mathrm{~h}$ en moyenne. Les symptômes ont persisté entre 3 à 21 jours et l'évolution a été spontanément favorable. Le premier cas de dermatite flagellaire par consommation de shiitake de cette série a été notifié en 2006 et $50 \%$ des cas (7 cas) ont été recensés depuis 2012.

Conclusion : Dans la littérature médicale une série de 51 cas de dermatites flagellaires a été décrite suite à l'ingestion de shiitake au Japon (Nakamura T, Contact Dermatitis. 1992). Le mécanisme serait toxique et est différent des allergies des travailleurs exposés aux cultures de shiitake (Tarvainen $\mathrm{K}$ et al., J Am Acad Dermatol. 1991). Un polysaccharide appelé lentinan entrant dans la composition du shiitake et utilisé pour sa propriété anti-cancéreuse serait à l'origine de cette toxidermie. En effet, les dermatites flagellaires sont une des complications observées lors de l'utilisation thérapeutique du lentinan. Il n'existe pas de traitement spécifique et les corticoïdes n'ont pas fait la preuve de leur efficacité. Ces champignons consommés habituellement en Asie sont désormais cultivés et commercialisés en France. Des recettes culinaires de shiitake crus ainsi que de produits à base de shiitake vantant ses vertus thérapeutiques fleurissent sur Internet. L'augmentation des cas depuis 2012, observée par les CAPTV, nécessite une alerte auprès des autorités de santé afin de prévenir le public du risque de toxidermie dû à une consommation de ces champignons crus ou peu cuits.

\section{C35. Lésions oculaires graves liées à l'Euphorbe : une intoxication accidentelle pas si rare...}

\section{E Ripley ${ }^{1}$, I Brisset ${ }^{2}$, M Boissonot ${ }^{3}$, F Penouil ${ }^{1}$, M Labadie $^{1}$}

${ }^{I}$ Centre antipoison et de toxicovigilance, CHU Hôpital Pellegrin, Bordeaux ; ${ }^{2}$ Centre antipoison et de toxicovigilance, CHU Hôpital Fernand Widal, Paris ; ${ }^{3}$ Service d'Ophtalmologie, $\mathrm{CHU}$, Poitiers

Objectif : Évaluer les risques d'intoxication oculaire liés aux projections oculaires d'Euphorbe, et leurs conséquences en terme de prise en charge.

Méthode : Nous avons rapporté les cas d'exposition de 2 patients suivis entre juin 2012 et septembre 2012 pour une intoxication oculaire à l'Euphorbe (Euphorbia lathyris dans un cas et Euphorbia Tommasiniana dans l'autre cas). Résultats : Les signes cliniques oculaires observés étaient similaires dans les 2 cas mais d'intensité variable, d'une simple gêne oculaire à la kératoconjonctivite, avec baisse de l'acuité visuelle. Dans le premier cas, après avoir immédiatement rincé l'œil à l'eau claire pendant 20 minutes et devant l'intensité des symptômes, le patient a consulté son médecin traitant qui a proposé un traitement symptomatique par collyre à la vitamine A. L'évolution a été favorable en $4 \mathrm{~h}$. Dans le second cas, le patient a bénéficié d'un avis ophtalmologique précoce puis d'un avis spécialisé au CHU, en raison de la gravité et la persistance des signes cliniques (réalisation de photos qui seront insérées dans le poster). Il était retrouvé une uvéo-kératite (ulcère central avec Tyndall +). Le traitement a consisté en un grattage superficiel associé à une injection sous-conjonctivale de corticoïdes, à l'instillation de collyre d'azithromycine, et de collyres mouillants. L'évolution a été favorable après 10 semaines de traitement.

Discussion et conclusion : Les euphorbiacées sont des plantes dont la toxicité est liée au latex contenant Diterpènes dérivés de l'ingénol et du 5 désoxyingénol (Crowder JL et al., Keratoconjonctivitis resulting from the sap of candelabra cactus and the pencil tree. Arch Ophthalmology. 1964). Au CAPTV de Bordeaux, nous avons recensé 10 cas d'exposition oculaire entre 2008 et 2012. Tous les patients étaient symptomatiques même pour des expositions minimes (frottement des yeux avec les doigts contaminés par exemple)(EKE, et al., The spectrum of ocular inflammation caused by Euphorbia plant sap. Arch Ophthalmology. 2000).

Au vu de ces 2 dossiers, des données des 5 dernières années (Glaizal M et al., Mediterranean Spurge (Genus Euphorbia) Poisonings: A Case Series from the Marseille Poison Centre, Clin Tox. 2011), il nous parait nécessaire d'insister sur la prise en charge précoce de ces plantes à latex irritant, avec lavage abondant et prolongé. Dans tous les cas, l'examen ophtalmologique avec test à la fluorescéine est essentiel pour repérer les lésions précocement et proposer la conduite à tenir (Shlamovitz GZ et al., A case of acute keratoconjunctivitis from exposure to latex of Euphorbia tirucali (pencil cactus). J Emer Med. 2009). Le premier patient a été suivi plusieurs mois en ophtalmologie avec in fine une évolution favorable et récupération complète de l'acuité visuelle. Le deuxième patient n'a pas nécessité de soins spécialisés ni de suivi.

\section{Nouvelles drogues, produits festifs}

\section{C36. Caractérisation d'une consommation de kava par analyse LC-MS/MS rapide de spots de sang séché (DBS)}

$\mathrm{C} \mathrm{Staub}^{1}, \mathrm{~F}_{\text {Versace }}{ }^{1}, \mathrm{Y}$ Barguil $^{2}$

${ }^{I}$ Centre Universitaire Romand de Médecine Légale, Unité de Toxicologie et Chimie Forensiques, Genève, Suisse ; ${ }^{2}$ Centre Hospitalier Territorial de Nouvelle-Calédonie, Nouméa, Nouvelle-Calédonie

Objectif : La boisson «kava» est obtenue par broyage et macération du rhizome de la plante Piper methysticum et consommée dans certaines îles du pacifique comme la Nouvelle-Calédonie. L'objectif principal est de caractériser une consommation de kava dans des microéchantillons de sang prélevés sur papier buvard à Nouméa et de déterminer la distribution des trois kavalactones principales : la kavaïne, la méthysticine et la yangonine à Genève après acheminement des échantillons par poste. Dans un deuxième temps, nous nous proposons d'évaluer l'influence de la distance d'acheminement, du mode de transport ainsi que de la durée de stockage avant l'analyse proprement dite.

Méthodes : L'étude a porté sur 29 cas postmortem datant de 2005 à 2012. Les échantillons de sang complet ont été stockés à $-20{ }^{\circ} \mathrm{C}$ jusqu'au moment de l'étude. $5 \mathrm{~mL}$ de sang ont été déposés à Nouméa sur un papier filtre type 903 proteinsaver de Whatman, puis ont été acheminés par la poste de Nouméa à Genève, dans un emballage hermétique contenant un sachet déshydratant. Les DBS ont été analysés sur la plateforme LC/MS/MS développée pour le screening (Versace et al., Chromatographia, 2013).

Résultats : La kavaïne a pu être caractérisée dans $96,6 \%$ des cas (28/29). Les concentrations mesurées sont comprises entre 14 et $5090 \mathrm{mg} / \mathrm{L}$ (moyenne : 588 et médiane : $214 \mathrm{mg} / \mathrm{L}$ ). La méthysticine, le deuxième composé actif, a été identifié dans 93,1\% des cas (27/29). Des concentrations de 12 à $1110 \mathrm{mg} / \mathrm{L}$ (moyenne : 198 et médiane : $75 \mathrm{mg} / \mathrm{L}$ ) ont été retrouvées. Enfin la yangonine, un composé inactif, a été mise en évidence dans 57,9\% des cas $(11 / 29)$ avec des concentrations variant de 58 à $1840 \mathrm{mg} / \mathrm{L}$ (moyenne : 
414 et médiane : $239 \mathrm{mg} / \mathrm{L}$ ). Ces concentrations sont dans la fourchette supérieure des teneurs retrouvées chez des consommateurs vivants et habituels de kava (Barguil, Thèse de doctorat, 2011).

Conclusion: L'approche DBS-LC/MS/MS nous a permis d'identifier les trois kavalactones (la kavaïne, la méthysticine et la yangonine) dans des microéchantillons de sang séché malgré la distance Nouméa-Genève, le mode de transport (envoi par la poste, courrier normal) et la durée de conservation avant le dépôt des spots de sang. De plus, cet envoi s'est fait de l'hémisphère sud en plein été austral vers l'hémisphère nord en plein hiver, avec des variations de température et d'humidité considérables. Finalement, ces résultats démontrent le potentiel des spots de sang séché pour des études à plus grande échelle où la distance entre le site de prélèvement et le laboratoire est importante.

\section{C37. Intoxications par les phénéthylamines, étude rétrospective au Centre antipoison et de toxicovigilance d'Angers}

G Le Roux ${ }^{1,3}$, A Turcant $^{2}$, D Boels ${ }^{3}$, C Bruneau ${ }^{3}$, M Bretaudeau ${ }^{3}$, B Lelievre ${ }^{2}$, S Ferec $^{2}$, P Harry ${ }^{3}$

${ }^{1}$ Service Pharmacie; ${ }^{2}$ Laboratoire de Pharmaco-toxicologie; ${ }^{3}$ Centre antipoison et toxicovigilance des régions Centre et Pays de la Loire; $\mathrm{CHU}$ d'Angers

Objectifs : Pour contourner la législation sur les stupéfiants, de nombreux produits appelés «nouveaux produits de synthèse » (NPS) apparaissent sur le marché des drogues dont ils «copient» les structures chimiques. Des phénéthylamines se rapprochant de la MDMA ou des amphétamines sont identifiées en France dès 2000 (4-MTA, 2C-B); les premières cathinones de synthèse sont identifiées en 2008 (méphédrone). En 2010, les phénéthylamines sont les NPS les plus représentées en Europe (Lahaie et al., Tendances. 2013). L'objectif de ce travail est de dresser un bilan rétrospectif des cas d'intoxications par les phénéthylamines recueillis pendant 6 ans par le CAPTV d'Angers.

Méthodes : L'interrogation sur Infocentre a été réalisée entre le $1^{\text {er }}$ janvier 2007 et le 31 décembre 2012 sur les cas du CAPTV d'Angers. Un cas est défini par toute personne exposée à un agent de la classe chimique des phénéthylamines dans un contexte de toxicomanie. Les critères de tri ont donc porté sur le libellé «Toxicomanie/Addiction » du champ "Circonstance » et sur les agents de la classe des phénéthylamines du champ «agent ». La gravité clinique a été définie selon le Poisoning Severity Score.

Résultats : Le CAPTV d'Angers a recensé 77 cas, provenant majoritairement des régions Centre et Pays de la Loire (43 cas dont 12 pour la seule LoireAtlantique), mais aussi de Bretagne (21 cas); 4 cas d'imputabilité nulle ont été exclus. Le sex-ratio (H/F) est de 3,70; l'âge moyen est de 25,2 \pm 8,2 ans. La consommation est principalement par voie orale (63,5\%). Dans 43 cas, une confirmation analytique a été possible. Pour les autres cas, il s'agissait principalement d'Ecstasy ou d'amphétamine et la confirmation analytique n'a pas été réalisée. Ecstasy (MDMA) (33 cas), amphétamine (15 cas) et méthamphétamine (11 cas) sont les plus consommées. La classe des $2 \mathrm{C}-\mathrm{X}$ (2C-P : 3 cas, $2 \mathrm{C}-\mathrm{E}: 1$ cas et $2 \mathrm{C}-\mathrm{T}-2: 1$ cas) et des cathinones (méphédrone : 3 cas, methcathinone : 4 cas, MDPV : 2 cas, méthylone : 1 cas ; 4-MEC: 1 cas) sont représentées. Dans près de la moitié des dossiers, la consommation est de type «monoagent». Dans les autres cas, le cannabis ( 21 cas), l'éthanol (16 cas) et la cocaïne ( 7 cas) sont les produits majoritairement associés aux phénéthylamines. La tachycardie est le symptôme le plus fréquent (10 cas) et l'hypertension artérielle dans les intoxications à l'amphétamine. Sur le plan psychiatrique et neurologique, les symptômes les plus courants sont : céphalées, paresthésies/hypoesthésie, angoisse/anxiété, agitation, et hallucinations. Hyperthermie $\left(38-41{ }^{\circ} \mathrm{C}\right)$ et hypersudation sont également retrouvées avec l'amphétamine et la méthcathinone. Les complications observées sont : convulsions (4 cas), arrêt cardiaque (4 cas), myocardite toxique (1 cas), AVC hémorragique (1 cas). Les patients ont été hospitalisés dans $80 \%$ des cas et 7 ont nécessité une prise en charge aux soins intensifs. Nous recensons 10 cas de gravité forte dont 4 décès et 1 séquelle. Pour tous ces cas de gravité forte, un dosage analytique spécifique a été demandé.

Conclusion : L'usage des phénéthylamines conduit à un tableau clinique assez homogène et typique : céphalées, tachycardie, et sur le plan psychiatrique, agitation, anxiété et fréquemment, des hallucinations. Les complications telles que convulsions, arrêt cardiaque, myocardite toxique, AVC assombrissent le tableau clinique (Simon, et al., L'information psychiatrique. 2003). Un dépistage urinaire de routine doit être réalisé précocement. Cependant, interférences, faux négatifs et informations imprécises sur les drogues (street names approximatifs, provenance incertaine, composition douteuse) imposent le recours à l'identification et au dosage du produit incriminé, par un laboratoire de toxicologie de référence devant toute intoxication grave ou inhabituelle. Les intoxications par les phénéthylamines génèrent un nombre croissant d'appels au CAPTV, et bien que banalisé sur Internet, l'usage des tels produits doit toutefois faire redouter des complications graves, parfois mortelles. Pour une veille efficace de ces nouvelles tendances de consommation, les échanges cliniciens/analystes sont indispensables.

\section{C38. Exposition aiguë à la 4-méthyl-éthylcathinone (4MEC), autre « nouvelle drogue de synthèse » accessible via Internet}

S Ferec ${ }^{1}$, D Boels ${ }^{2}$, M Bretaudeau ${ }^{1}$, B Lelievre ${ }^{1}$, I Leborgne ${ }^{1}$, P Harry ${ }^{1}$, B Diquet ${ }^{1}$, A Turcant ${ }^{1}$

${ }^{1}$ Laboratoire de pharmacologie et Toxicologie, CHU Angers; ${ }^{2}$ Centre antipoison - Toxicovigilance Angers, $\mathrm{CHU}$ Angers

Objectifs : La 4-méthyl-éthylcathinone (4-MEC), molécule proche de la méphédrone et de la pentédrone, fait partie de la famille des cathinones récemment classée en 2012 comme produits stupéfiants par l'Agence Nationale de Sécurité du Médicament. Une analyse clinico-biologique est présentée pour un homme de 39 ans après exposition intraveineuse à cette substance.

Cas clinique : Un patient toxicomane s'injecte en IV vers 18h (H0), à l'aide d'un kit Stéribox ${ }^{\circledast}$, entre 0,25 et $0,5 \mathrm{~g}$ de poudre achetée sur un site web hollandais sous le nom 4-MEC. Il présente les effets recherchés euphorisants et désinhibants. Puis il inhale vers $21 \mathrm{~h} 30$ un aérosol d'une bombe de froid à usage médical à base de chlorure d'éthyle. Sous l'effet euphorisant toujours intense de la 4-MEC, le patient décide d'ingérer du GBL (produit de nettoyage de jantes fourni par un ami) qu'il surdose par rapport à ses consommations précédentes. Dans les 30 min suivantes, son compagnon le retrouve inconscient. Aux urgences, vers $23 \mathrm{~h}$, le patient présente des pauses respiratoires nécessitant une stimulation et une oxygénation. L'examen clinique retrouve une FC à $81 \mathrm{bpm}$, une TA à 120/80 mmHg, des pupilles intermédiaires réactives et l'ECG est sinusal. À 23h30, le patient se réveille mais reste confus. Les propos deviennent cohérents $4 \mathrm{~h}$ après son réveil. Le bilan biologique à $\mathrm{H} 8$ est sans particularité. L'alcoolémie est nulle. Un bilan toxicologique plasmatique et urinaire à $\mathrm{H} 8$ est également effectué. La poudre de 4-MEC a également été analysée. Le patient est sortant le lendemain à $12 \mathrm{~h}$. Au suivi à 3 mois le patient va bien.

Matériel et méthodes : Une recherche large de médicaments et toxiques a été réalisée par CPG-SM et CLHP-UV-BD. Les amphétamines et le GHB sont analysés par CPG/SM après dérivation par HFBA ou silylation. Une méthode quantitative par CLUHP-SM/SM a également été développée sur $100 \mu \mathrm{L}$ de plasma (ou urine diluée $\geq 1 / 20^{\mathrm{e}}$ ) avec simple précipitation des protéines par le méthanol $(150 \mu \mathrm{L})$ puis dilution au 1/4 dans l'eau avant injection $(5 \mu \mathrm{L})$. La colonne (Acquity UPLC $^{\circledR}$ BEH C18 $1,7 \mu \mathrm{m}$ $2,1 \times 50 \mathrm{~mm}$ ) est maintenue à $40{ }^{\circ} \mathrm{C}$. La phase mobile, méthanol et formiate d'ammonium $2 \mathrm{mM}(+0,1 \%$ acide formique), varie de $20 / 80(0,5 \mathrm{~min})$ à $90 / 10$ en 2,5 $\mathrm{min}(0,5 \mathrm{~mL} / \mathrm{min})$. L'acquisition s'effectue en mode MRM (Multiple Reaction Monitoring) après ionisation ES+ avec 2 transitions $(192>145,1$ et $192>159,1)$ et une pour l'EI $(261.3>142.8)$. La méthode a été validée sur le plasma à 3 concentrations différentes $(15,300$ et $750 \mu \mathrm{g} / \mathrm{L}$; $\mathrm{n}=4$ sur 4 jours). 
Résultats : Le dépistage urinaire des amphétamines est positif. La méthode $\mathrm{SM} / \mathrm{SM}$ est linéaire de 10 à $1000 \mu \mathrm{g} / \mathrm{L}\left(\mathrm{r}^{2}>0.994\right)$. La justesse $\left(0,2 \%<\mathrm{I} \%_{\text {moye }}\right.$ $<6,0 \%)$, la répétabilité $\left(\mathrm{CV}_{\mathrm{r}}<7,5 \%\right)$ et la reproductibilité $\left(\mathrm{CV}_{\mathrm{FI}}<7,9 \%\right)$ sont satisfaisantes. La limite de quantification est fixée et validée à $10 \mu \mathrm{g} / \mathrm{L}$. Les concentrations observées chez le patient sont de $353 \mu \mathrm{g} / \mathrm{L}$ pour le plasma et $100 \mathrm{mg} / \mathrm{L}$ pour l'urine. Le GHB plasmatique et urinaire est égal à 300 et $1000 \mathrm{mg} / \mathrm{L}$. Plusieurs pics chromatographiques présentant des analogies spectrales avec $4 \mathrm{MEC}$ sont mis en évidence et peuvent correspondre à des métabolites.

Conclusion : Ce cas clinique, avec évolution favorable en raison d'une prise en charge rapide, illustre l'utilisation de plus en plus fréquente de produits en accès libre sur Internet et les risques de polyconsommation induite, dans le cas présent, par la 4-MEC. Aucune donnée de concentration de 4-MEC n'a été retrouvée dans la littérature.

\section{C39. La 5 APB et la 5 MAPB, nouvelles alternatives échappant à la législation sur les excitants en milieu festif ?}

\section{F Aknouche $^{1}$, E Guibert $^{1}$, M Eyraud ${ }^{1}$, K Fargeot $^{1}$, P Kintz $^{2}$}

${ }^{1}$ Laboratoire BIO6MED, Antibes ; ${ }^{2} X$-Pertise Consulting, Oberhausbergen

Introduction : De plus en plus citées dans les congrès scientifiques, de nouvelles molécules ne cessent de faire leur entrée dans le marché des substances récréatives. Pour échapper à la réglementation internationale ou nationale, les laboratoires clandestins et les trafiquants synthétisent des molécules soit par ajout soit par suppression d'un ou plusieurs radicaux sur des structures existantes. Classer ces substances comme stupéfiant prend un certain temps, et par conséquent ces nouvelles substances peuvent échapper à toute règlementation. Par ailleurs, s'en procurer reste un jeu d'enfant grâce à Internet, d'où la nécessité et le rôle pour les laboratoires experts de pouvoir les identifier et de communiquer! Nous rapportons ici le cas d'une saisie de poudre blanche par les forces de gendarmerie dans la région des AlpesMaritimes. Contenus dans trois sachets hermétiques, ces quelques grammes de poudre pouvaient laisser penser à une saisie d'amphétamine.

Méthodes : Les investigations scientifiques mises en œuvre pour qualifier la poudre contenue dans chaque scellé ont commencé par l'application de la procédure de screening classique utilisée lors d'expertise toxicologique de poudre. Cette analyse est réalisée par chromatographie en phase gazeuse (modèle FOCUS GC, ThermoElectron, Courtabœuf, France), couplée à un spectromètre de masse de type trappe d'ions (modèle POLARIS Q, ThermoElectron, Courtabœuf, France). En parallèle, un rapide test immunologique (automate de type AXSYM, Abbott, Rungis, France), a été réalisé pour détecter la présence éventuelle de cocaïniques ou d'amphétaminiques par simple dilution de la poudre dans de l'eau distillée. De plus, une méthode de chromatographie en phase gazeuse (modèle TRACE GC, ThermoElectron, Courtabœuf, France), couplée à un détecteur de type spectrométrie de masse (modèle TSQ 8000, ThermoElectron, Courtabœuf, France) a été mise en place afin d'identifier les ions fils des ions majoritaires détectés lors du screening chromatographique.

Résultats : Concernant la technique immunologique, cette molécule a donné une valeur supérieure à $8000 \mathrm{ng} / \mathrm{mL}$ pour la détection du groupe des amphétamines. L'analyse de cette poudre en GC-MS a révélé la présence dans les scellés 1 et 2 d'une molécule avec deux ions majoritaires $\mathrm{m} / \mathrm{z}$ suivants : 131, 44 et dans le scellé 3 d'une molécule avec trois ions majoritaires $\mathrm{m} / \mathrm{z}$ suivants : 58, 131, 190. Cependant, il demeure un ion présent dans chaque étude, à savoir le $\mathrm{m} / \mathrm{z}=77$. La méthode de spectrométrie de masse en tandem effectuée après dérivation des poudres par un agent dérivant de type HFBA (Heptafluorobutyric Acid - Macherey-Nagel), a donné les résultats suivants : $\mathrm{m} / \mathrm{z} 371>158, \mathrm{~m} / \mathrm{z} 240>192, \mathrm{~m} / \mathrm{z} 240>169$ pour les poudres contenues dans les sachets 1 et 2 et m/z $386>158, \mathrm{~m} / \mathrm{z} 210>131$ dans le sachet 3 .

Conclusion: Les ions spécifiques trouvés sur les spectres des poudres analysées semblent correspondre à la fragmentation des structures chimiques mentionnées dans la littérature des deux poudres identifiées
5-APB (5-(2-amino-propyl) benzofuran-5-ylpropan-2-amine) et 5-MAPB (1-(benzofuran-5-yl)-N-méthyl-2-amine). Cependant aucune substance de référence n'est aujourd'hui disponible. Cette identification présente deux nouvelles molécules analogues de la MDA aux effets excitants et entactogènes, non référencées comme stupéfiant actuellement en France.

\section{C40. Décès par noyade après consommation de « NRG-3 » : rôle des cathinones?}

M Loilier $^{1}$, P Laburthe-Tolra ${ }^{2}$, D Debruyne ${ }^{1}$, F Papin-Lefebvre $^{2}$, A Coquere $^{1}$ ${ }^{1}$ Laboratoire de Pharmacologie-Toxicologie, CHU, Caen, France ; 2 Institut de Médecine Légale, CHU, Caen, France

Objectifs : La 3,4-methylenedioxypyrovalérone (MDPV), la pyrrolidinovalérophénone (PVP) et l'éthylmethcathinone (pentédrone) sont trois molécules synthétiques dérivées de la cathinone appartenant à la famille des «nouvelles drogues». Ces trois molécules sont accessibles sur Internet notamment sous les appellations «NRG-2 », «NRG-3». Ces appellations sont des mélanges de plusieurs cathinones. Les effets pharmacologiques sont de type amphétamine-like avec troubles neurologiques, neuropsychiques et cardiovasculaires avec possibilité de survenue d'infarctus du myocarde et d'arrêt cardio-respiratoire. Nous rapportons un cas de décès par noyade d'un jeune homme ayant consommé des quantités importantes de «NRG-3 » contenant un mélange de 3 cathinones : MDPV, PVP et pentédrone.

Description du cas : Un jeune homme de 27 ans est retrouvé noyé dans un port, près d'un ponton, tête en bas touchant le fond à la verticale. L'examen de corps révèle un syndrome asphyxique avec une cyanose en pèlerine et met en évidence un sachet non identifié dans la poche du pantalon contenant une poudre blanche. Des échantillons biologiques sont adressés au laboratoire pour analyse toxicologique ainsi que le sachet de poudre pour identification. Parallèlement à ces premiers échantillons biologiques, un second sachet de poudre identifié « NRG-3 » nous a été fourni provenant du domicile du sujet. Méthodes : Un dépistage urinaire de toxiques est effectué en immunochimie. Des recherches larges par méthodes séparatives de substances toxiques et médicamenteuses sont également faites sur les urines, par chromatographie gazeuse couplée à un spectromètre de masse (GC/MS) et par chromatographie liquide couplée à un spectromètre de masse en tandem (LC/MS/MS), après extraction liquide-liquide. Les dosages sanguins quantitatifs spécifiques des molécules identifiées sont réalisés par différentes méthodes chromatographiques. Les deux sachets de poudre sont identifiés par chromatographie gazeuse couplée à un spectromètre de masse (GC/MS).

Résultats : Les analyses toxicologiques ont révélé la présence de MDPV, PVP et pentédrone dans les échantillons biologiques ainsi que dans les 2 poudres mais avec des proportions différentes :

\begin{tabular}{|l|c|c|c|c|}
\cline { 2 - 5 } \multicolumn{1}{c|}{} & Poudre 1 (\%) & Poudre 2 (\%) & Sang $(\boldsymbol{\mu g} / \mathbf{L})$ & Urines $(\boldsymbol{\mu g} / \mathbf{L})$ \\
\hline MDPV & 17 & 24 & 106 & 760 \\
\hline PVP & 6 & 8 & 40 & 295 \\
\hline Pentédrone & 46 & 10 & 33 & 110 \\
\hline
\end{tabular}

De l'hydroxyzine, du nordazepam, de l'oxazepam et du THC-COOH ont été également mis en évidence aux concentrations sanguines de $194 \mu \mathrm{g} / \mathrm{L}$, $47 \mu \mathrm{g} / \mathrm{L}, 8 \mu \mathrm{g} / \mathrm{L}$ et $15,7 \mu \mathrm{g} / \mathrm{L}$ respectivement ; associés à une consommation d'alcool (sang : 0,3 g/L ; urines : 1,3 g/L).

Conclusion: L'analyse toxicologique a montré une consommation de plusieurs cathinones associée à une prise d'hydroxyzine, de cannabis, de benzodiazépines et d'alcool, ayant pu potentialiser les effets toxiques des cathinones. Le MDPV a été retrouvé en quantité importante, de l'ordre des concentrations pouvant entraîner un malaise cardiaque ayant pu provoquer la chute puis la noyade (Murray et al., J Med Toxicol. 2012). Il n'y a pas eu d'autopsie pour confirmer cette hypothèse. 
C41. Identification et dosage de la 4-MEC (4-méthyléthylcathinone)etdelaMDPV (3,4-méthylènedioxypyrovalérone) dans différents milieux : à propos d'un cas

\author{
A Knapp $^{1}$, L Humbert ${ }^{2}$, I Etting ${ }^{1}$, E Abe $^{1}$, R Garnier $^{3}$, JC Alvarez ${ }^{1}$ \\ ${ }^{1}$ Pharmacologie/Toxicologie, CHU R Poincaré, APHP, Garches et Université \\ Versailles St Quentin en Yvelines ; ${ }^{2}$ Toxicologie, $\mathrm{CHU}$ Lille ; ${ }^{3} \mathrm{CAP} C H U$ \\ Fernand Widal, Paris
}

Objectif : Un homme de 30 ans consulte pour obtenir une aide au sevrage de la consommation habituelle de cathinones, qu'il se procure sur internet. Il déclare consommer de façon régulière des stupéfiants depuis l'adolescence : du cannabis depuis 15 ans ( 1 à 2 « joints » par semaine), de la cocaïne depuis deux ans (en moyenne 1 gramme par week-end), qu'il dit avoir interrompu quand il a commencé à consommer des cathinones ; épisodiquement, de la MDMA depuis une dizaine d'années. Il aurait débuté sa consommation de cathinones depuis 6 mois. Il se fournit sur internet et achète généralement de la 4-MEC ou du NRG3, rarement du NRG2. Il ne consomme habituellement que pendant les week-ends. Il s'administre les produits par voie IV, à la dose d'un quart à une demie gélule toutes les 45 à 90 minutes. Jusqu'à ces dernières semaines, sa consommation hebdomadaire moyenne se limitait à 5-7 injections, mais il l'a récemment augmentée et a été tenté de consommer aussi pendant la semaine. Le week-end précédant la consultation, il a consommé 5 grammes de NRG3 et $10 \mathrm{~g}$ de 4-MEC du vendredi soir au dimanche soir. Il a eu une bouffée délirante et une tachycardie qui ont motivé un bref passage aux urgences de son hôpital de secteur dans la nuit du dimanche au lundi. Il consulte le mercredi matin. Il ne se plaint plus de rien. Son examen clinique ne montre pas d'autre anomalie qu'un petit hématome au pli du coude droit. Des échantillons de sang, d'urines et de cheveux sont prélevés ainsi qu'un échantillon de la poudre consommée pour identifier et caractériser sa consommation réelle de cathinones.

Méthode : Des méthodes de screening par HPLC-BD, GC-MS et LC-MS/MS sont mises en œuvre sur les échantillons de sang, d'urine et sur la poudre. Devant la présence de composés non identifiés dans la poudre et dans les urines, ces échantillons sont également analysés sur UPLC-QTOF (Waters ${ }^{\circledR}$ ). La quantification des molécules identifiées sur l'ensemble des milieux (y compris les cheveux de 1-2 cm de long) est réalisée par LC-MS/MS (TSQ Vantage ThermoFischer ${ }^{\circledR}$ ) par une méthode validée. Les différents milieux biologiques subissent une extraction liquide/liquide par un mélange hexane/ acétate d'éthyle (1/1, v/v) en présence de tampon carbonate $1 \mathrm{M}, \mathrm{pH}$ 9,7. La séparation est réalisée sur une colonne Hypersyl gold PFP $(200 \times 2,1 \mathrm{~mm}$, $1,9 \mu \mathrm{m})$, l'élution est obtenue par un gradient d'acétonitrile et de tampon formiate $2 \mathrm{mM}, 0,1 \%$ d'acide formique. L'ionisation est réalisée en mode ESI+ et l'identification en mode MRM.

Résultats : Du paracétamol et du tramadol sont retrouvés dans le plasma aux concentrations respectives de $7 \mu \mathrm{g} / \mathrm{mL}$ et $465 \mathrm{ng} / \mathrm{mL}$. Dans les urines, on retrouve ces deux molécules associées à de la MDMA $(20 \mathrm{ng} / \mathrm{mL})$, de la MDPV $(20 \mathrm{ng} / \mathrm{mL})$ et de la 4-MEC $(200 \mathrm{ng} / \mathrm{mL})$. L'analyse par UPLCQTOF permet d'identifier certains métabolites de ces deux composés. La poudre est identifiée comme étant de la 4-MEC dont la pureté est de $50 \%$. Dans les cheveux dosés en vrac sont retrouvés de la 4-MEC (30 ng/ $\mathrm{mg}$ ), de la MDPV (1 ng/mg), de la MDMA et MDA (2 et $0,1 \mathrm{ng} / \mathrm{mg}$ ), de la méphédrone $(0,1 \mathrm{ng} / \mathrm{mg})$, de la cocaïne $(1,7 \mathrm{ng} / \mathrm{mg})$ et métabolites (BZE $=0,2$ et $\mathrm{EME}=0,02 \mathrm{ng} / \mathrm{mg}$ ) mais aussi du tramadol $(3,5 \mathrm{ng} /$ $\mathrm{mg})$, de l'hydroxyzine $(0,14 \mathrm{ng} / \mathrm{mg})$, de l'aripiprazole $(11 \mathrm{ng} / \mathrm{mg})$ et de l'halopéridol $(0,01 \mathrm{ng} / \mathrm{mg})$.

Conclusion : Nous rapportons ici un premier cas d'identification et de dosage de 4-MEC associée à de la MDPV dans différentes matrices et en particulier les cheveux, avec caractérisation de certains métabolites.

\section{Toxicologie médico-judiciaire}

\section{C42. Interpretation of the toxicological findings in a Red Bull? related fatality}

JM Gaulier ${ }^{1}$, C Coutaz ${ }^{2}$, B Magret $^{2}$, L Conjat ${ }^{2}$, T Chianea ${ }^{3}$, F Sturtz ${ }^{3}$, S Dulaurent ${ }^{1}$, L Imbert ${ }^{2}$, F Paraf $^{2}$, G Lachatre $^{1}$

${ }^{1}$ Toxicology laboratory, ${ }^{2}$ Forensic medicine, ${ }^{3}$ Biochemistry laboratory, $\mathrm{CHU}$, Limoges

Objective: Today, energy drinks (ED) are frequently used or abused in private, sporting, or festive contexts. There are regular reports on the risk of repeated intakes of such brewages. However, documented issues related to the adverse health consequences of ED remain to be clarified. In this unclear situation, we propose to report a fatality including Red Bull? intakes, and the difficulties in the interpretation of the toxicological findings.

Case history: At an overnight party held in a house, a 36 year-old man with a recent acute pericarditis antecedent, was in charge of the music (DJ). Witnesses reported an overnight consumption by this man of alcohol, cannabis and energy drinks (Red Bull?). At the end of the night, he complained of thoracic pain, and went outside to "take a breath of fresh air": one hour later, he was found dead outside the house, on the pavement. Autopsy findings showed a cyanosis linked to an acute pulmonary oedema, together with adhesive pericarditis. The collected biological samples [including peripheral blood (PB), urine (UR), vitreous humour $(\mathrm{VH})$ and gastric content (GC)] were sent to the laboratory for forensic toxicological analysis.

Methods: Large screenings of drugs and toxicants were performed in post-mortem blood and urine samples large screening of drugs and toxics compounds in both $\mathrm{PB}$, UR and GC was performed using gas chromatography - mass spectrometry (GC-MS) and liquid chromatographytandem mass spectrometry (LC-MS/MS). More selective analyses were carried out with various ad-hoc methods using LC and GC with various detection modes. So, blood caffeine determination was performed using a LC method with diode array detection (LC-DAD), and a previously reported method was used for taurine determination in whole blood, using a LC-DAD method, and including a pre-treatment step consisting in three consecutive defecations of blood using trichloracetic acid [1].

Results: The observed PB concentrations of caffeine and taurine were $0.51 \mathrm{mg} / \mathrm{L}$ and $984 \mu \mathrm{mol} / \mathrm{L}$, respectively. The positive results are presented in the following table.

\begin{tabular}{|l|c|c|c|c|}
\hline \multirow{2}{*}{ Ethanol } & \multicolumn{2}{|c|}{ Ethylglucuronide } & \multicolumn{2}{c|}{ Cannabinoides } \\
\cline { 2 - 5 } & & THC & THC-COOH & $11-\mathrm{OH}$ THC \\
\hline $0.79 \mathrm{~g} / \mathrm{L}(\mathrm{PB})$ & $194 \mathrm{mg} / \mathrm{L}(\mathrm{UR})$ & $<1 \mu \mathrm{\mu g} / \mathrm{L}(\mathrm{PB})$ & $4.1 \mu \mathrm{g} / \mathrm{L}(\mathrm{PB})$ & $<1 \mu \mathrm{g} / \mathrm{L}(\mathrm{PB})$ \\
$0.65 \mathrm{~g} / \mathrm{L}(\mathrm{VH})$ & & & $>100 \mu \mathrm{g} / \mathrm{L}(\mathrm{UR})$ & $7.4 \mu \mathrm{g} / \mathrm{L}$ (UR) \\
$0.77 \mathrm{~g} / \mathrm{L}(\mathrm{UR})$ & & & & \\
$0.65 \mathrm{~g} / \mathrm{L}(\mathrm{GC})$ & & & & \\
\hline
\end{tabular}

Conclusion: The toxicological findings are coherent with ethanol and cannabis consumption in the few hours before the decease. At the same time, the PB concentrations of caffeine and taurine (about three times over the maximal physiological values for taurine in whole blood [Trautwein et al., Am J Clin Nutr. 1990J) are in agreement with the hypothesis of ED intake(s) during the same period. To our knowledge, this elevated post-mortem PB taurine concentration cannot be related to any toxic effect. The adverse effects of ED can be related to either the toxicity of ingredients (especially, caffeine), or to the combination of the ingredients, or to specific situations in which energy drinks are used such as ingestion in combination with alcohol, or cardiological pre-dispositions [Reissig et al., Drug Alcohol Depend. 2009]. As a consequence and in this situation, the toxicological implication of the ED-Alcohol intakes could only be suggested, and not established. 


\section{C43. Double premeditated infanticide by administration of zopiclone}

$\underline{\text { AL Pelissier-Alicot }}{ }^{1}$, P Kintz ${ }^{2}$, M Deveaux ${ }^{3}$, C Sastre $^{1}$, V Baillif-Couniou ${ }^{1}$, G Leonetti ${ }^{1}$

${ }^{1}$ Service de Médecine Légale, Faculté de Médecine, Marseille; ${ }^{2} X^{\prime} P e r t i s e$ Consulting, Oberhausbergen; ${ }^{3}$ Laboratoire Toxlab, Paris

Introduction: Zopiclone is a hypnotic agent belonging to a class of nonbenzodiazepine hypnosedative drugs, the cyclopyrrolones. Zopiclone is prescribed in the treatment of occasional insomnia in adults and doesn't have any official French market authorization for children. Some offlabel prescriptions have been described in adolescents (Mancini $\mathrm{J}$ et al., Pharmacoepidemiol Drug Saf. 2006) but none in children. Fatal poisonings are mainly represented by suicides, essentially in elderly and/or in patients with an incurable disease, often in combination with other drugs (Bramness GJ et al., J Forensic Sci. 2001). To our knowledge, no deaths have been reported in children. We present here a double infanticide with premeditation by zopiclone administration and drowning.

Description: Mrs. X, 38 years old, called the emergency services explaining she had found her children lifeless in the bathtub. The paramedics found the two children, G 2 years old, and R, 5 years old, dead in the bathtub full of water. Suspicions quickly turned to the mother who rapidly confessed to having drowned her children after drugging them with zopiclone. Autopsies were performed 48 hours later and revealed lung and brain edema and a small amount of water in the lungs and stomach of the eldest. No sign of abuse or injury in self- defense was noted. Samples were taken for conventional toxicological analysis (heart and femoral blood, gastric content, urine, vitreous humor and hair). An analysis of maternal hair was also performed to confirm the alleged use of psychotropic drugs by the mother during interrogation.

Methods: Benzodiazepines, Z-drugs (zolpidem and zopiclone), neuroleptics and other sedatives were determined in peripheral blood and urine by liquid chromatography coupled to tandem mass spectrometry (LC-MS/MS) after liquid-liquid extraction in Toxitubes A, and in hair by LC-MS/MS after liquid-liquid extraction.

Results: Results obtained from samples of the victims and their mother are presented in the tables 1 and 2:

Table 1:

\begin{tabular}{|l|c|c|c|c|}
\cline { 4 - 5 } \multicolumn{1}{c|}{} & \multirow{2}{*}{$\begin{array}{c}\text { Peripheral } \\
\text { blood } \\
(\mathbf{n g} / \mathbf{m L})\end{array}$} & \multirow{2}{*}{$\begin{array}{c}\text { Urine } \\
(\mathbf{n g} / \mathbf{m L})\end{array}$} & $\begin{array}{c}\text { Segment 1 (4 cm) } \\
\text { 09/2009_01/2010 }\end{array}$ & $\begin{array}{c}\text { Segment 2 (4 cm) } \\
\text { 05/2009_09/2009 }\end{array}$ \\
\hline Victim G & Zopiclone: 260 & Zopiclone: 1580 & Zopiclone: 16 & Zopiclone: 12 \\
\hline Victim R & Zopiclone: 174 & Zopiclone: 946 & Zopiclone: 45 & Zopiclone: 40 \\
\hline
\end{tabular}

Table 2:

\begin{tabular}{|c|c|c|c|c|}
\hline & \multicolumn{4}{|c|}{ Hair (pg/mg) } \\
\hline & $\begin{array}{l}\text { Segment } 1(3 \mathrm{~cm}) \\
09 / 2009-01 / 2010\end{array}$ & $\begin{array}{l}\text { Segment } 2(3 \mathrm{~cm}) \\
05 / 2009-09 / 2009\end{array}$ & $\begin{array}{c}\text { Segment } 3(3 \mathrm{~cm}) \\
05 / 2009-09 / 2009\end{array}$ & $\begin{array}{l}\text { Segment } 4(3 \mathrm{~cm}) \\
05 / 2009-09 / 2009\end{array}$ \\
\hline \multirow{4}{*}{ Mother } & Zolpidem: 1,7 & Zolpidem: 18 & Zolpidem: 10 & Zolpidem: 2,5 \\
\hline & Zopiclone: - & Zopiclone: 18 & Zopiclone: 45 & Zopiclone: 54 \\
\hline & Cyamemazine: 61 & Cyamemazine: 53 & Cyamemazine: 44 & Cyamemazine: 41 \\
\hline & Amitriptyline: 1568 & Amitriptyline: 1236 & Amitriptyline: 772 & Amitriptyline: 627 \\
\hline
\end{tabular}

No reference values exist for zopiclone in children. The post-mortem blood concentrations found in the victims correspond to toxic concentrations in adults. The presence of very low concentrations of zopiclone in all segments of hair shows regular exposure of the two victims during the 8 months preceding the death. The analysis of maternal hair confirms a regular intake of benzodiazepine hypnotics, as cyamemazine and amitriptyline. The mother was found responsible for her acts by expert psychiatrists and sentenced to 25 years imprisonment. $\overline{\text { C44. Application of a physiologically based pharmacokinetic }}$ modelisation to forensic toxicology: the case of citalopram tissular distribution

N Cartiser $^{1,2}$, F Bevalot $^{1,2}$, M Tod $^{2,3}$, J Guitton $^{2,3}$

${ }^{1}$ Laboratoire LATLUMTOX, ${ }^{2}$ Université Lyon $1,{ }^{3}$ Hospices civils de Lyon, Lyon

Aim: The aim of this study was to evaluate the interest of Physiologically Based PharmacoKinetic (PBPK) modelisation in forensic toxicology to interpret concentrations in alternative matrices. In complement of data issued of case reports, standardized animal experiments are required to describe tissular kinetic. However, results of such experiments need to be extrapolated to human and to various situations (e.g. administration route and pattern, pathology...). PBPK modelisation is a powerful tool for theoretical representation of whole body pharmacokinetic (PK) and for the extrapolation of PK data. This approach has been widely used in pharmaceutical development and in ecotoxicology but not in the forensic toxicology field. We applied PBPK modelisation to explore the human citalopram tissular distribution from animal experiments.

Methods: Animal experiments were performed on 25 New Zealand rabbits which received $2.8 \mathrm{mg} / \mathrm{kg}$ of Seropram ${ }^{\circledR}$ intravenously. Animals were sacrificed $2 \mathrm{~h}, 4 \mathrm{~h}, 7 \mathrm{~h}, 17 \mathrm{~h}$ and $32 \mathrm{~h}$ after administration (5 rabbits per time). Lung, kidney, liver, bone marrow (BM), brain, adipose tissue, skeletal muscle and central blood were collected and citalopram was quantified by gas chromatography coupled to tandem mass spectrometry (assay validated for each matrix). PBPK was built in three steps. First, the model was constituted for rabbit with mathematical equation corresponding to most of PK process (Levitt, BMC Clin Pharmacol. 2003), rabbit physiological data from literature, blood and tissue concentrations issued of animal experiments. Then, cross-validation was performed by comparing simulated kinetic to experimental one. The last step was the extrapolation to human by modifying physiological parameters from rabbit to human. PBPK modelisation was processed on Adapt II software (Biomedical Simulations Resource, University of Southern California, USA).

Results: PBPK modelisation was successfully applied to simulate human blood and tissue distribution of citalopram after oral administration from animal experiments (rabbits, intravenous route). Concordance between simulated kinetics and experimental ones is excellent $\left(\mathrm{r}^{2}>0.86\right.$ and up to 0.996 in blood and tissues). Extrapolated blood kinetic in human, characterized by an elimination half-life of $13.5 \mathrm{~h}$ and a peak concentration at $68 \mathrm{~min}$, fits to data reported in literature although slightly faster. Distribution and elimination have been modelised in each studied tissue.

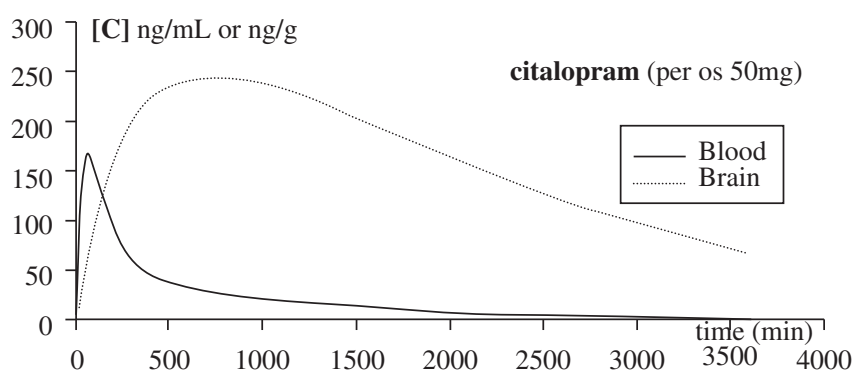

Citalopram kinetic in blood and brain simulated by PBPK modelisation for a $50 \mathrm{mg}$ oral administration.

Conclusion: This study highlights the interest of PBPK approach in forensic toxicology. This approach enables to transpose animal experiments to human, to simulate various administration route and pattern and to explore whole body tissular kinetic. Simulate kinetics for tissues of forensic interest can be a powerful help to interpret toxicological results obtained on these alternative matrices. 


\section{C45. Cyamemazine bile concentration more informative than blood concentration? A study from 340 post-mortem observations}

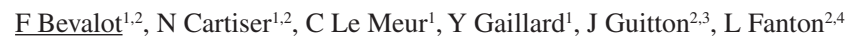

${ }^{1}$ Laboratoire LATLUMTOX, ${ }^{2}$ Université Lyon 1, Lyon; ${ }^{3}$ Hospices Civils de

Lyon, Pierre Bénite; ${ }^{4}$ Institut Médico-Légal de Lyon, Lyon

Aim: The aim of this presentation is to demonstrate the interest of measuring and interpreting cyamemazine bile concentrations. In a preliminary study, performed on 22 observations, we proposed, when blood sample is lacking, a diagnostic test to interpret cyamemazine bile concentration (Bévalot et al., $\mathrm{XII}^{\mathrm{e}}$ Congrès SFTA, Porticcio. 2004). A bile concentration threshold was evaluated at $10 \mu \mathrm{g} / \mathrm{mL}$ above which overdose and so potentially intoxication could be hypothesized. This threshold was considered as equivalent to a $0.5 \mu \mathrm{g} / \mathrm{mL}$ threshold in blood. We have extended this study to 340 postmortem observations involving cyamemazine to evaluate the diagnostic value of the proposed threshold and to improve knowledge on concentrations measured in this matrix.

Methods: Since 2004, all cases in which cyamemazine has been detected in at least one matrix collected during autopsy (blood, gastric content and/or urine) and from which blood and bile were available were included. Blood samples were collected and analyzed from femoral vein or/and cardiac cavity according to availability. Analyses were performed by GC/MS after liquid/liquid extraction. Cases were classified, after comparison of conclusions obtained from bile and blood concentrations, as: True Positive: [blood] $>0.5 \mu \mathrm{g} / \mathrm{mL}$, [bile] $>10 \mu \mathrm{g} / \mathrm{mL}$; True Negative: [blood] $<0.5 \mu \mathrm{g} / \mathrm{mL}$, [bile] $<10 \mu \mathrm{g} / \mathrm{mL}$; False Positive: [blood] $<0.5 \mu \mathrm{g} / \mathrm{mL}$, [bile] $>10 \mu \mathrm{g} / \mathrm{mL}$; False Negative: [blood] $>0.5 \mu \mathrm{g} / \mathrm{mL}$, [bile] $<$ $10 \mu \mathrm{g} / \mathrm{mL}$. The diagnostic value of the test has been evaluated by calculating its sensitivity (SE) and its specificity (SP).

Results: 340 cases with various causes of death and postmortem delays were included in this study. Based on blood threshold, 274 were considered as therapeutic and 55 as overdosed. Interpreting with the $10 \mu \mathrm{g} / \mathrm{mL}$ threshold in bile, there were 255 true negative, 36 true positive, 19 false negative and 19 false positive cases. The resulting intrinsic qualities of the test were: $\mathrm{SE}=0.65$ and $\mathrm{SP}=0.93$. The 19 false positive cases have been fully investigated from a medico-legal point of view (police reports, autopsy observations, full toxicological investigation...): 10 could be considered as toxic death implicating cyamemazine, 2 have another cause of death and for 7 cases, circumstances could not be defined by lack of information. In the 11 remaining cases, there was a discordance of interpretation between peripheral and cardiac blood samples. Among them, a suicide by drug overdose implicating cyamemazine was very likely in 6 cases. The corresponding bile concentrations were superior to $10 \mu \mathrm{g} / \mathrm{mL}$.

Conclusion: Interpretation of cyamemazine bile concentration allows to contribute to answer at the question: "Has the decedent absorbed a therapeutic or a supra-therapeutic dose of cyamemazine?". This interpretation should be used in different situation: lack of blood samples, discordance between blood concentrations and more generally in each situation where toxic death implicating cyamemazine could not be formally ruled out. Three cases will be developed during presentation to illustrate those various situations.

C46. Quetiapine: now available in French pharmacies, already in your autopsy cases!

B Brunet, M Lebeau, P Decourt, S Macia, P Mura

Laboratoire de Toxicologie et Pharmacocinétique, Centre Hospitalier Universitaire, Poitiers

Introduction: We report here 2 cases of death where quetiapine a newly available antipsychotic was involved. Quetiapine is an atypical antipsychotic that has been approved for the market in France and commercialized in November 2011. This medication is prescribed for schizophrenia and bipolar disorders. Quetiapine was available under temporary authorisation for use
(ATU) since 2006 in France and is marketed in most of European countries and the US. Quetiapine is supposedly one of the most well tolerated atypical antipsychotic.

Methods: 2 cases of suicide are described. The first case involved a 60-yearold woman who committed suicide by ingestion of various drugs. At the autopsy no traumatic lesion was found and the cause of death suspected was drug overdose. The second case involved a 59-year-old woman who committed suicide by drowning. Complete toxicological screenings were carried out on both cases. It included identification and/or quantification of alcohols, and drugs of abuse (cannabinoids, opiates, cocaine, amphetamines). Presence of drugs was investigated by means of liquid chromatography coupled to photodiode array detection or tandem mass spectrometry.

Results: Quetiapine was found in both cases. First case revealed a mixeddrug intoxication with multiple psychotropic drugs namely: zopiclone, bromazepam, oxazepam, alprazolam, lormetazepam, lorazepam, cyamemazine and quetiapine. Among those drugs only zopiclone was found at a toxic concentration. In the second case quetiapine was found along with a low concentration of zopiclone. As quetiapine is a newly released product in France, thorough investigations of its concentrations in the different biological matrices available was carried out. For case 1, quetiapine concentrations were $108 \mathrm{ng} /$ $\mathrm{mL}$ and $5 \mathrm{ng} / \mathrm{mL}$ respectively for peripheral blood and vitreous humor. For case 2 , concentrations were $166 \mathrm{ng} / \mathrm{mL}, 212 \mathrm{ng} / \mathrm{mL}$, and $7 \mathrm{ng} / \mathrm{mL}$ respectively for peripheral blood, cardiac blood and vitreous humor. Hair specimen was analyzed and gave a value of $4,15 \mathrm{ng} / \mathrm{mg}$.

Discussion: In the two cases described here the blood concentrations of quetiapine were not lethal. From the analytical point of view, a heart blood/ femoral blood ratio of 1.31 has been reported, which is consistent with our finding in case 2. Vitreous humor concentrations in both cases were low showing a poor diffusion of quetiapine in this biological fluid. In the second case the cause of death was drowning, but in the first case the association of quetiapine with other SNC depressants might have had a played a role in the process of death. Physicians and patients are informed by the manufacturer that there is an increased risk of suicide when taking quetiapine and that they should consult if they are feeling more depressed and have thoughts about suicide.

Conclusion: Quetiapine is a newly released product and like any other antipsychotic it has a potential for abuse and might be present in forensic cases. Toxicologists should update their screening procedures to include such new molecules. Moreover quetiapine like other antidepressants and antipsychotics, at some point of the treatment, could favour suicide gesture among patients. Those cases should therefore be monitored by poison centres and drug safety authorities.

\section{C47. A fatal sniff of lidocaine}

C Duverneuil-Mayer $^{1}$, A Knapp ${ }^{1}$, G Lorin de la Grandmaison ${ }^{2,3}$, JC Alvarez ${ }^{1}$ ${ }^{1}$ Laboratory of pharmacology-toxicology, ${ }^{2}$ Institut of Legal Medicine, CHU $R$ Poincaré, Garches; ${ }^{3}$ University of Versailles St Quentin en Yvelines

Case: A 27-years-old man was discovered at home in his bathroom. His neighbor would have heard him prepare a bath. He heard a deaf sound that could be a fall. White powder with equipment dedicated to sniff was found near him at home. None cause of death was found at autopsy.

Objective: The aim of this study was to determine the composition of the powder and to evaluate its implication in the cause of death.

Methods: Several samples were taken at autopsy and brought to our laboratory: blood, urines, gastric liquid, bile and vitreous humor. Secondary, the powder discovered at his home was brought to the laboratory by the Police. An urinary screening of drugs of abuse was realized by immunoanalysis (Axsym, Abbott ${ }^{\circledR}$ ), with confirmation of the positive results by gas chromatography coupled to mass spectrometry (GC-MS). A nonspecific research or "screening" was done in blood, urines and gastric liquid by GC-MS and liquid chromatography coupled to diode array detection 
(LC-DAD) after liquid/liquid extraction in acidic and basic conditions. Specific research methods by GC-MS, LC-DAD or LC-MS/MS (liquid chromatography coupled to tandem mass spectrometry detection) were done for psychotropic substances. An alcohol dosage was realized by GC-FID on all the samples at our disposal. Concerning the powder, a screening by GC-MS and LC-DAD as well as specific researches of drugs of abuse were realized. Lidocaine and MEGX were analyzed with a validated LC-MS/MS method.

Results: Alcohol was not found in any sample. Concerning drugs of abuse, only tetrahydrocannabinol (active principle of cannabis) was measured in concentration of $1.2 \mathrm{ng} / \mathrm{mL}$ in blood with its principal metabolite, THC$\mathrm{COOH}$, in blood concentration of $5 \mathrm{ng} / \mathrm{mL}$ and urinary concentration of $52 \mathrm{ng} / \mathrm{mL}$. No other psychotropic drug was found. Only lidocaine and its metabolite MEGX were detected in samples. For lidocaine and MEGX LC/MS/MS method, linearity was validated in the $0.2-5 \mu \mathrm{g} / \mathrm{mL}$ range for lidocaine and $0.02-0.5 \mu \mathrm{g} / \mathrm{mL}$ range for MEGX. Extraction recovery was about $92 \%$ for lidocaine and $70 \%$ for its metabolite. The limits of detection (LOD) were about $0.11 \mu \mathrm{g} / \mathrm{mL}$ for lidocaine and $0.01 \mu \mathrm{g} / \mathrm{mL}$ for MEGX and limits of quantification (LOQ) were respectively $0.2 \mu \mathrm{g} / \mathrm{mL}$ and $0.02 \mu \mathrm{g} / \mathrm{mL}$. Intra-day and inter-day CVs evaluated at two concentrations levels were all lower than $10 \%$ with accuracy between $93 \%$ and $102 \%$. Matrix effect was insignificant. The concentrations of lidocaine and MEGX detected in blood of this case report were very high $(13.6 \mu \mathrm{g} / \mathrm{mL}$ and $80 \mu \mathrm{g} / \mathrm{mL}$, respectively). Therapeutic blood concentrations of lidocaine and its metabolite are in the $1-5 \mu \mathrm{g} / \mathrm{mL}$ and $0.07-0.175 \mu \mathrm{g} / \mathrm{mL}$ range, respectively. Toxic blood concentration of lidocaine was defined between 6 and $10 \mu \mathrm{g} / \mathrm{mL}$, and lethal blood concentration was greater than $10 \mu \mathrm{g} / \mathrm{mL}$. So, in our case, it was concluded that lidocaine and its metabolite were responsible for the death. In the powder, no drug of abuse was highlighted, and its composition was $100 \%$ of lidocaine.

Conclusion: It seems that thinking he was consuming cocaine, this man who was a dealer died by a sniff of a lidocaine powder that he usually used to cut his cocaine powder. So, this example shows that a product cutting can be fatal when administered in place of the drug of abuse substance. The implication of the product of cutting in the deaths by overdose was recently evoked in the litterature (Barbera N et al., FSI. 2013).

\section{$\overline{\text { C48. Sampling and preparation of textile samples in forensic }}$ toxicology}

L Imbert $^{1}$, JM Gaulier ${ }^{1}$, A Baudoin ${ }^{1}$, C Conte ${ }^{1}$, S Dulaurent ${ }^{1}$, V DumestreToulet $^{2}$, P Kintz ${ }^{3}$, G Lachatre ${ }^{1}$

${ }^{1}$ Toxicology and Pharmacology unit, CHU Dupuytren, Limoges; ${ }^{2}$ ToxGen, Bordeaux; ${ }^{3} \mathrm{X}$-Pertise Consulting, Oberhausbergen

Objectives: Sampling non-biological samples can sometimes be challenging in forensic toxicology. While sampling of various solid materials (powders, tablets, food...) is frequent, toxicological analyses of textile samples (sheets, clothes...) are rare. In order to document this real issue, we present three forensic cases for which this type of matrix was provided, in order to propose a process for sample preparation.

Cases stories: Case 1 consisted in a pyjama with multiple bloodstains, with a request for toxicological screening, including specific tests for psilocin, tetrahydrocannabinol (THC) and LSD. Case 2 concerned a motorcycle helmet with a request for a specific test for glyphosate. Case 3 presents a sheet stained with vomit, with a request for toxicological screening and a specific test for methadone and sugar.

Methods: Case 1: The pyjama was divided into four parts which were all sampled (posterior high and low, anterior high and low) that were extracted separately by $2.5 \mathrm{~mL}$ water and $12.5 \mathrm{~mL}$ methanol. Case 2 : The inner coating of the helmet was divided into seven zones, subsequently extracted by water. Case 3: The area of the sheet stained with vomit was cut and directly extracted by methanol. In all of these 3 forensic cases, the samples were agitated and sonicated for two hours. After centrifugation, the liquid phase was then evaporated and resuspended before injection in the solvent corresponding to the analytical method used. A screening and the analysis of THC in case 1 were performed by GC-MS, and another screening and the specific tests for psilocin and LSD were performed using LC-MS/MS (Sauvage et al., J Sep Sci. 2009). In case 2, a LC-MS/MS specific method was performed for glyphosate determination (Zouaoui et al., Forensic Sci Int. 2013). In case 3, a GC-MS screening was performed, together with a specific assay for methadone using GC-MS.

\section{Results:}

\begin{tabular}{|l|l|l|l|}
\hline Case & 1 & 2 & 3 \\
\hline \multirow{5}{*}{ Results (presence of) } & Nicotine & & Methadone and \\
& Cafein & & EDDP \\
& Theophylline & Glyphosate & Glycerol \\
& Lidocaine & & Glucose, saccharose \\
& Tiaptride & & Sorbitol \\
Cafein, nicotine
\end{tabular}

Conclusion: These 3 cases highlight the importance of the pre-analytical step, which is as important as the analytical process; therefore sampling must be carefully planned. In our cases, liquid extraction of xenobiotics from textile matrices was well performed with methanol or a water/methanol mixture. Such an extraction only makes it possible to give a qualitative interpretation to these different forensic cases.

\section{Cannabis}

\section{C49. THC et CBD capillaires et troubles psychiatriques chez le consommateur de cannabis}

$\underline{\text { Y Barguil }}^{1,2}$, JY Charlot ${ }^{3}$, F Baumann ${ }^{4}$, E Pain ${ }^{1}$, J Cousin ${ }^{1}$, E Choblet ${ }^{1}$, L Lepot $^{1}$, M Nour ${ }^{2}$, G Southwell ${ }^{3}$

${ }^{1}$ Laboratoire de Biochimie, CHT-NC ; ${ }^{2}$ Université de la Nouvelle-Calédonie, Nouméa ; ${ }^{3} \mathrm{CHS}-\mathrm{NC}$, Nouméa ; ${ }^{4}$ University of Hawaii Cancer Center, USA

Objectif : Dans une précédente étude, nous avons montré que le ratio des concentrations delta-9-THC/CBD capillaires était fréquemment élevé chez les consommateurs de cannabis schizophrènes (Barguil et al., Actes du $18^{\mathrm{e}}$ congrès de la SFTA. 2010). Elle montrait aussi que la pharmacopsychose au cannabis survenait, le plus souvent, chez de «faibles » consommateurs qui pouvaient présenter une susceptibilité individuelle. Poursuivant ce travail, nous avons étudié les concentrations capillaires en THC et en CBD de 112 patients consommateurs de cannabis.

Méthodes: Quatre groupes de consommateurs de cannabis, confirmés positifs et consentants ont été déterminés selon les critères du DSM IV-TR (plus de 6 mois de suivi psychiatrique). Groupe 1:36 patients hospitalisés pour raisons chirurgicales ou médicales (groupe témoin). Groupe 2: 35 patients bénéficiant d'un suivi spécialisé pour le traitement de Troubles Psychiatriques Chroniques du registre Psychotique (TPCP), majoritairement schizophréniques. Groupe 3: 20 patients ayant présenté une psychose aiguë cannabique (PA). Groupe 4:21 patients présentant des troubles psychiatriques autres (états limites, dépression...). À partir de prélèvements capillaires, le THC et le CBD ont été dosés par LC-MS² (LOQ : 0,005 ng/ $\mathrm{mg}$ ) sur la totalité du prélèvement. Les pourcentages ont été comparés par le test du chi ${ }^{2}$ de Pearson, ou le test exact de Fisher. Les moyennes ont été comparées par le t-test, sinon par le U-test non paramétrique de WilcoxonMann-Whitney.

Résultats : Résumés dans le tableau ci-dessous (moyennes \pm écart-type). 


\begin{tabular}{|l|c|c|c|c|c|c|}
\hline Groupes & $\begin{array}{c}\text { Nombre } \\
\text { de sujets }\end{array}$ & Âge (années) & $\%$ femmes & THC ng/mg & CBD ng/mg & $\begin{array}{c}\text { Ratio } \\
\text { THC/CBD }\end{array}$ \\
\hline Témoins & 36 & $26,9 \pm 7,6$ & $22,2 \%$ & $0,496 \pm 0,949$ & $0,062 \pm 0,121$ & $37 \pm 109$ \\
\hline TPCP & 35 & $27,6 \pm 7,1$ & $14,3 \%$ & $0,956 \pm 1,407$ & $0,059 \pm 0,137$ & $129 \pm 245$ \\
\hline PA & 20 & $29,0 \pm 6,9$ & $30,0 \%$ & $0,042 \pm 0,081$ & $0,002 \pm 0,002$ & $9 \pm 16$ \\
\hline Autres & 21 & $28,6 \pm 9,4$ & $47,6 \%$ & $0,181 \pm 0,203$ & $0,019 \pm 0,042$ & $25 \pm 31$ \\
\hline
\end{tabular}

Le groupe TPCP présente des concentrations capillaires en THC et un ratio significativement plus élevés que le groupe témoin ( $\mathrm{p}=0,046$ et 0,045 respectivement) et des concentrations capillaires en THC significativement plus élevées que le groupe Autres Troubles ( $p=0,006$ ).

Comparé au Groupe témoin, le groupe Psychose Aiguë cannabique présente des concentrations capillaires en THC et en CBD très significativement plus faibles ( $\mathrm{p}=0,0085$ et 0,020 , respectivement) et un ratio comparable. Si l'on compare le groupe Autres Troubles au groupe PA cannabique, les valeurs de tous les paramètres sont significativement plus faibles pour le groupe PA ( $\mathrm{p}=0,004 ; 0,022$ et 0,046 pour THC, CBD et THC/CBD, respectivement). Si l'on compare le groupe TPCP au groupe PA cannabique, les valeurs de tous les paramètres sont significativement plus faibles pour le groupe PA ( $\mathrm{p}=0,0001 ; 0,0016$ et 0,0062 pour THC, CBD et THC/CBD, respectivement).

Conclusions : Malgré la petitesse des échantillons résultant en des intervalles de confiance importants, l'étude des 3 valeurs THC, CBD et du ratio THC/CBD capillaires permettrait de discriminer les patients consommateurs de cannabis souffrant d'une psychose chronique des patients présentant une psychose cannabique ou des consommateurs souffrant d'un autre trouble psychiatrique. De même chez les consommateurs, il serait possible de distinguer les patients souffrant d'une psychose cannabique des patients souffrant d'un autre trouble. Cette étude se poursuit afin d'améliorer la précision de nos valeurs moyennes dans chaque population.

\section{C50. Cannabis et accident cardiaque aigu : à propos de 2 observations issues de la médecine légale}

$\underline{\text { A Boucher }}{ }^{1}$, C Bidat ${ }^{2}$, T Basset $^{3}, \mathrm{~J}_{\text {Descotes }}{ }^{1}, \mathrm{~S}_{\text {Duband }}^{2}$

${ }^{I}$ Centre Antipoison-Centre d'Addictovigilance, Hospices Civils de Lyon;

${ }^{2}$ Service de Médecine Légale et ${ }^{3}$ Laboratoire de Pharmacologie Toxicologie, CHU de Saint-Étienne

Objectifs : Au cours de la dernière décennie, plusieurs cas d'accidents cardiaques aigus peu après une consommation de cannabis ont été rapportés dans la littérature médicale, incluant des ischémies myocardiques et des morts subites. Après avoir décrit deux cas de morts subites au décours d'une consommation de cannabis, nous passerons en revue les hypothèses étiopathologiques actuellement avancées.

Méthode: Nous présentons deux cas de mort subite au décours d'une consommation de cannabis chez des patients jeunes et sans antécédent cardiaque connu. Dans les deux cas, une autopsie avec examen anatomopathologique a été pratiquée à la recherche des causes de la mort. Les analyses toxicologiques dans le sérum ont été réalisées par GC-MS et LC-MS/MS. L'alcoolémie a quant à elle été déterminée par GC-FID.

Cas cliniques : Le premier patient, âgé de 41 ans, est décédé environ 2 heures après avoir présenté un malaise à son domicile avec douleur thoracique. L'autopsie a permis de conclure à un décès par ischémie myocardique aiguë en dépit d'artères coronaires saines, avec consommation de cannabis peu avant le décès $(\mathrm{THC}=4,6 \mathrm{ng} / \mathrm{mL}$ et THC-COOH $=18,7 \mathrm{ng} / \mathrm{mL}$ dans le sang). Des stigmates d'épisodes ischémiques plus anciens ont également été relevés chez cet homme. Le second patient, âgé de 42 ans, est retrouvé à son domicile plusieurs jours après son décès. L'autopsie n'a retrouvé aucune lésion aiguë susceptible d'expliquer le décès mais a mis en évidence une cicatrice d'infarctus du myocarde ancien. Seuls des cannabinoïdes ont été identifiés dans le sang, à des concentrations compatibles avec une consommation très récente par rapport au décès $(\mathrm{THC}=52,7 \mathrm{ng} / \mathrm{mL}$ et
THC-COOH = 5,8 ng/mL dans le sang). Là encore, l'absence d'anomalie coronarienne pose la question du mécanisme de survenue du décès.

Discussion : Plusieurs hypothèses sont avancées dans la littérature afin d'expliquer ces accidents cardiaques aigus : spasme coronarien, déséquilibre entre tonus sympathique et parasympathique, déséquilibre entre demande et apport en oxygène vers le myocarde, troubles du rythme à l'origine de mort subites (Lindsay et al., Int J Cardiol. 2005 ; Caldicott et al., Eur J Emerg Med. 2005 ; Pratap et al., Cardiovasc Toxicol. 2012 ; Safaa et al., Drug Alcohol Rev. 2012). Par ailleurs, un lien chronologique entre le déclenchement d'un syndrome coronarien aigu et le moment de la consommation de cannabis a été mis en évidence par l'équipe de Mittleman, avec un risque de survenue multiplié par 5 dans l'heure suivant la consommation (Mittleman et al., Circulation. 2001).

Conclusion : L'implication du cannabis dans la survenue d'accidents cardiaques aigus a été avancée dans plusieurs cas publiés, généralement comme diagnostic d'exclusion après enquête étiologique adéquate. Son rôle précis dans la genèse de ces accidents - rares malgré une consommation répandue - et les mécanismes de survenue restent mal compris. Néanmoins, nos deux observations rappellent que cette hypothèse étiologique doit être discutée en cas de décès chez un sujet jeune, devant un bilan toxicologique évocateur et en l'absence d'autre cause clairement identifiée.

\section{C51. Hyperémèse cannabique et élimination prolongée du THC : nos connaissances remises en question !}

P Mura $^{1}$, B Brunet ${ }^{1}$, A Lebrun $^{2}$, C Chagneau-Derrode ${ }^{2}$

${ }^{1}$ Service de toxicologie et pharmacocinétique, CHU, Poitiers; ${ }^{2}$ Service d'hépato-gastro-entérologie, $\mathrm{CHU}$, Poitiers

Objectif : Dans certains pays, le cannabis est proposé pour lutter contre les douleurs, les nausées et les vomissements. Devant un tableau clinique comprenant des douleurs abdominales violentes, des nausées et des vomissements coercitifs, la recherche de cannabis dans les urines apparaît donc être au prime abord sans intérêt. Le cas décrit dans la présente observation montre que dans ce contexte clinique, une simple recherche de cannabis dans les urines associée à un interrogatoire approprié peut permettre d'éviter de nombreuses explorations voire des hospitalisations répétées.

Patient et méthodes : Mme M, 28 ans, est admise dans le service d'hépatogastro-entérologie du CHU de Poitiers. Elle a été hospitalisée 14 fois au cours des 13 derniers mois dont 7 séjours de 5 à 12 jours. Comme à chaque épisode d'hospitalisation, elle se plaint de douleurs abdominales violentes, de nausées et de vomissements évoluant par crises. Pendant toute cette période, une perte de poids de $33 \mathrm{~kg}$ a été observée. Chaque crise commencait par une douleur diffuse secondairement localisée dans la région épigastrique, nécessitant très fréquemment une administration de morphine à l'hôpital. Lors de chaque séjour hospitalier, les symptomes régressaient progressivement pour finalement disparaître, sans traitement antalgique.

Résultats : Au cours des 13 derniers mois, toutes les causes connues par l'équipe médicale comme pouvant être responsables d'une telle symptomatologie ont été recherchées, sans succès. Les bilans immunologique, endocrinien et infectieux sont négatifs. Les explorations morphologiques digestives et cérébrales sont normales. Suite à une recherche urinaire positive pour le cannabis à $\mathrm{J} 6$, des échantillons sanguins de J4, J5 et J7 sont analysés et conduisent respectivement aux résultats suivants en $\mathrm{ng} / \mathrm{mL}$ : THC $(1,7 ; 2,4$ et 1,1$), 11-\mathrm{OH}-\mathrm{THC}(1,1$; $1,4$ et 0,7$)$ THC-COOH (131; 47 et 23). L'interrogatoire de la patiente sur ce sujet rapporte une consommation règulière et importante de cannabis, d'environ 6 « joints » par jour et la cessation complète de toute consommation depuis son admission. L'analyse des cheveux confirme cette importance de consommation et l'absence de cotinine dans les urines confirme l'abstinence de consommation de joints. Elle déclare également que la prise de douches chaudes calme immédiatement les douleurs et les nausées.

Discussion : «L'hyperémèse cannabique-syndrome de la douche chaude » a été initialement décrite en 2004 par une équipe australienne et une 
centaine de cas ont été décrits depuis 2009. Cette pathologie concerne des consommateurs réguliers et importants de cannabis. Son diagnostic repose sur l'existence de douleurs abdominales avec des vomissements, une anorexie, une polydipsie, une asthénie intense, des sueurs froides voire une hypothermie. La prise d'antalgiques ou de tout autre traitement symptomatique n'apporte aucune amélioration significative. Seul un bain ou une douche chaude atténue notablement douleurs, nausées et vomissements. Même si certaines hypothèses sont avancées, on ne connaît pas à ce jour les mécanismes d'action impliqués dans cette hyperémèse paradoxale. La méconnaissance de cette pathologie par le corps médical est responsable d'un sous-diagnostic, induisant des hospitalisations itératives, des explorations nombreuses et coûteuses et surtout un retard de la prise en charge pouvant être considérable, comme dans le cas présent. Par ailleurs, la persistance de concentrations significatives en THC et 11-OH-THC dans le sang pendant une semaine voire plus bouleverse nos connaissances dans ce domaine et confirme des travaux très récents réalisés chez des consommateurs réguliers et importants de cannabis (Bergamaschi MM et al., Clin Chem. 2013).

\section{COMMUNICATIONS AFFICHÉES}

\section{P1. Du diagnostic psychiatrique au diagnostic toxicologique : l'analyste au secours du clinicien}

\section{A Garat $^{1,2}, \mathrm{C} \mathrm{Richeval}^{1}, \mathrm{~F}_{\text {Tison }}^{3}$, D Allorge ${ }^{1}, \mathrm{P} \mathrm{Nisse}^{2}$}

${ }^{1}$ Laboratoire de Toxicologie, Centre de Biologie Pathologie, CHRU, Lille ; ${ }^{2}$ Centre Antipoison, CHRU, Lille ; ${ }^{3}$ Service d'accueil Urgences-SMUR, CH, Armentières

Objectif : Le Centre Antipoison de Lille a été contacté pour un patient de 38 ans adressé aux urgences pour une symptomatologie évocatrice d'une bouffée délirante aiguë. Dans ses antécédents, on note un éthylisme chronique et deux épisodes délirants, dont l'un était consécutif à une prise avouée de kétamine. La biologie à l'entrée montre une rhabdomyolyse (CPK : 4400 UI/L, myoglobine plasmatique : $165 \mathrm{ng} / \mathrm{mL}$ ). Le bilan toxicologique réalisé sur place n'a pas permis de détecter d'éthanol, de barbituriques, de benzodiazépines ou d'antidépresseurs tricycliques dans le sérum. La famille du patient a retrouvé dans sa chambre deux sachets vides de substances commandées sur le site http://chemicalservice.net/ et portant les indications (1-(5-fluorophenyl)-1Hindol-3-yl)-1-naphthalenylmethanone, un cannabinoïde de synthèse également appelé MAM-2201, et methoxyphenyl ethylamine cyclohexanone, plus connue sous le nom de méthoxétamine, un dérivé de la kétamine. Ce travail rapporte la méthodologie et les résultats des analyses toxicologiques, réalisées en complément du bilan d'entrée, qui ont permis d'expliquer les signes cliniques présentés par ce patient.

Méthodes : Des échantillons de sérum et d'urines ont été envoyés au laboratoire de toxicologie du CHRU de Lille pour analyses complémentaires. Les urines, préalablement hydrolysées par une $\beta$-glucuronidase, et le sérum ont été extraits à $\mathrm{pH}$ acide et à $\mathrm{pH}$ alcalin avec un mélange de solvants organiques et en présence d'étalons internes. Le screening toxicologique a été réalisé par UPLC-G2 QTOF (Waters). La séparation chromatographique a été réalisée sur une colonne HSS $\mathrm{C}_{18} 1,7 \mu \mathrm{m}, 150 \mathrm{~mm} \times 2.1 \mathrm{~mm}$ à l'aide d'un gradient de tampon formate d'ammonium $\mathrm{pH}$ 3/acétonitrile à $0,1 \%$ d'acide formique. L'acquisition a été effectuée en mode $\mathrm{MS}^{\mathrm{E}}$ en electrospray positif et le traitement des données réalisé grâce au logiciel POSIT \pm VE.

Résultats : La prise en charge du patient a consisté en une sédation par diazépam à raison de $10 \mathrm{mg}$ toutes les $4 \mathrm{~h}$ par voie IV et en une surveillance neurologique et cardiaque. Le lendemain de son hospitalisation, son état clinique rassurant a permis son transfert en service de psychiatrie. L'analyse toxicologique a permis de confirmer la prise de méthoxétamine, retrouvée dans le sérum à la concentration de $70 \mathrm{ng} / \mathrm{mL}$, accompagnée de ses métabolites desméthylé et deséthylé. D'autre part, de l'hydroxyzine et du tétrazépam ont été retrouvés à des concentrations sériques de 600 et $140 \mathrm{ng} / \mathrm{mL}$, leurs métabolites ayant également été détectés. L'analyse des urines a permis de mettre en évidence la présence des métabolites de la méthoxétamine, de l'hydroxyzine et du tétrazépam. En revanche, le MAM2201 n'a pas été détecté dans ces deux matrices.

Conclusion : La détection de méthoxétamine et de ses métabolites dans les milieux biologiques de ce patient a permis d'imputer ce syndrome délirant à une étiologie toxique. La valeur de la concentration sérique de $70 \mathrm{ng} / \mathrm{mL}$ est comparable aux valeurs précédemment décrites dans des cas d'intoxications non létales (Ferec et al., Ann Toxicol Anal. 2012; Abe et al., Ann Toxicol Anal. 2012; Wood et al., Eur J Clin Pharmacol. 2012). Le criblage toxicologique a également mis en évidence une prise associée, non avouée, d'hydroxyzine et de tétrazépam. Ce cas illustre la nécessité actuelle de disposer d'outils analytiques permettant la mise en évidence de designer drugs, très facilement accessibles via Internet.

\section{$\overline{\text { P2. Accessibilité des antidotes en urgence : exemple du CHU }}$ de Tizi-Ouzou}

LR Mekacher, H Ouardene, S Ya

Laboratoire de Biochimie-Toxicologie, $\mathrm{CHU}$, Tizi-Ouzou, Algérie

Objectif : Le but de notre étude est d'évaluer la disponibilité réelle et l'emploi des antidotes dans le Centre Hospitalo-Universitaire de Tizi-Ouzou. Méthodes : Nous avons évalué, au niveau de la pharmacie centrale du CHU, la disponibilité de certains antidotes, sélectionnés selon la fréquence de l'intoxication ou bien la pertinence du traitement. Par la suite, un questionnaire, permettant d'apprécier l'utilisation de ces antidotes, a été adressé au personnel médical des services des urgences médicales, des urgences pédiatriques et de la réanimation médicale.

Résultats : Sur les 12 antidotes sélectionnés, 8 n'étaient pas inscrits sur la nomenclature nationale des médicaments et seulement 3 étaient disponibles au niveau de la pharmacie centrale du CHU. Il faut signaler que certains antidotes non inscrits sur la nomenclature sont disponibles au niveau de la pharmacie du CHU. La N-acétylcysteine (Fluimucil ${ }^{\circledR}$ ) par voie orale est inscrite sur la nomenclature mais n'est pas disponible au CHU. Les cliniciens ont précisé que l'antidote est acheté à titre externe par les malades sous forme de sachets. L'antidote par voie injectable n'est pas inscrit sur la nomenclature. $94 \%$ des cliniciens déclarent donner de l'atropine en cas d'intoxication par les pesticides organophosphorés alors que la pralidoxime (Contrathion ${ }^{\circledR}$ ) est disponible au niveau du CHU. $52 \%$ des cliniciens déclarent ne pas utiliser du charbon actif comme traitement évacuateur. Dans le cas ou il est utilisé, il est acheté par le malade à titre externe.

Conclusion : En raison du coût très élevé de certains antidotes, de la rareté des intoxications pour lesquelles ils sont utilisés et de leur courte durée de conservation, il est souvent impensable pour les centres hospitaliers de maintenir des quantités suffisantes de tous les antidotes susceptibles d'être requis à un moment donné. Une solution consiste à sélectionner les antidotes les plus susceptibles d'être utiles et d'établir des ententes avec les autres centres hospitaliers environnants afin d'être en mesure de se procurer ou de fournir rapidement un antidote en cas d'urgence.

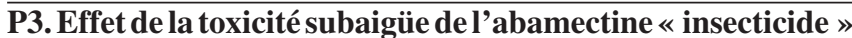 sur le foie des rats (Rattus norvegicus)}

H Khaldoun-Oularbi ${ }^{1}{ }^{1,2}, \mathrm{C} \mathrm{Richeval}^{3}$, N Djenas ${ }^{5}$, M Lhermitte $^{3,4}$, L Humbert $^{3}$ ${ }^{1}$ Département de Biologie, Faculté des Sciences Agrovétérinaires et biologiques, Université SAAD-Dahlab Blida, Algérie; ${ }^{2}$ Laboratoire de recherche de Biologie et Physiologie Animale, ENS, Kouba, Algérie; ${ }^{3}$ Laboratoire de Toxicologie, CHRU, Lille ; ${ }^{4}$ Université de Lille ; ${ }^{5}$ Laboratoire d'Anatomie Pathologie CHRU Parnet, Alger

Objectif : L'utilisation intensive de pesticides a des effets néfastes sur la santé humaine et l'environnement (Dallegrave E et al., Arch Toxicol. 2007; 
Colosio C \& Moretto A. International Encyclopedia of Public Health. 2008). L'abamectine (ABM) a été largement utilisée partout dans le monde (Turner MJ \& Schaeffer JM, Mode of action of Ivermectin. Springer Verlag. New York. 1989) et est l'un des insecticides les plus utilisés en Algérie. L'empoisonnement par l'abamectine peut altérer la fonction des hépatocytes (Castanha Zanoli JC et al., Toxicol In Vitro. 2012).

Méthodes : Afin d'évaluer les effets toxiques de l'ABM sur la fonction hépatique, vingt-huit rats mâles et femelles «Rattus norvegicus» ont été répartis au hasard en quatre groupes. Deux groupes témoins, mâle et femelle, recevaient de l'eau distillée ( $1 \mathrm{~mL} / \mathrm{rat} / \mathrm{jour})$. Deux groupes traités, mâle et femelle, recevaient $2,13 \mathrm{mg} / \mathrm{animal} / \mathrm{jour}$ d'abamectine administrée par voie orale pendant 28 jours. Les animaux ont été maintenus dans les mêmes conditions sans traitement pendant 14 jours après cette période. Les échantillons de plasma aux $14^{\mathrm{e}}, 28^{\mathrm{e}}$ et $42^{\mathrm{e}}$ jours ont été utilisés pour déterminer les paramètres biochimiques et les résidus de l'abamectine B1a. Les quantités de $\mathrm{B} 1 \mathrm{a}$ dans le foie ont été évaluées à la fin de l'expérience (jour 42). Dans cette étude, une méthode UHPLC-MS/MS a été utilisée pour déterminer les résidus de l'abamectine dans le plasma et le foie.

Résultats: L'abamectine a provoqué une augmentation $(\mathrm{p}<0,005)$ de la glycémie et des enzymes ASAT, ALAT et $\gamma$-GT chez les rats mâles et femelles. Les résidus de l'abamectine ont été détectés dans le plasma de tous les rats traités à j14 et j28 alors qu'à la fin de l'expérimentation une absence totale de B1a a été constatée. Dans le foie, à j42, B1a a été détecté à différentes concentrations chez les rats traités $(83 \mathrm{ng} / \mathrm{g}$ pour les mâles et $362 \mathrm{ng} / \mathrm{g}$ pour les femelles). Les résultats histopathologiques montrent que l'abamectine a provoqué des changements dans l'histologie du foie à savoir une dilatation des veines, une infiltration des leucocytes et une dégénérescence des hépatocytes.

Conclusion : Nos résultats montrent que l'ABM perturbe la fonction hépatique chez le rat.

\section{P4. Rapid and sensitive quantitation of THC and metabolites in Urine by Microflow LC-MS/MS}

\author{
D Blake $^{1}$, A Morla $^{1}$, S Dulaurent ${ }^{2}$, J.-M Gaulier ${ }^{2}$, J Moriceau ${ }^{1}$ \\ ${ }^{I}$ ABSciex, Les Ulis; ${ }^{2}$ Laboratoire de Pharmacologie-Toxicologie, $\mathrm{CHU}$ de \\ Limoges
}

Objective: Rapid turnaround and high sensitivity for urine drugs of abuse analysis is necessary in a toxicology laboratory. Additionally, as financial and environmental concerns become more prevalent, heavy use of solvents for high flow LC-MS/MS methods, can be an issue. We present a rapid, robust, sensitive \& cost-effective method for the simultaneous quantitative analysis of Tetrahydrocannabinol (THC) and its major metabolites hydroxytetrahydrocannabinol (THC-OH) and carboxy-tetrahydrocannabinol (THC$\mathrm{COOH}$ ) in urine using sample dilution and exploiting Microflow LC for the dramatic increases in throughput and on column sensitivity, reduced solvent consumption and system robustness this technique offers.

Method: The sample preparation consists of a simple pre-treatment step using dilution of urine samples, followed by direct injection. HPLC methodology consists of a water/methanol/buffer mobile phase with a short gradient on a $0.5 \mathrm{~mm}$ ID Microflow LC column, using Microflow LC. Mass Spectrometry analysis was carried out running MRM, giving quantitative results for the THC and metabolites in urine.

Results: Using this method it was possible to analyse the THC and metabolites in urine with an injection - injection time of 3 minutes. Mean sensitivity for all compounds is shown to be $<0.5 \mathrm{ng} / \mathrm{mL}$ in urine. This equates to $<500 \mathrm{fg}$ on column sensitivity for these compounds. Mean robustness has been demonstrated and shows accuracies within $10 \%$ of nominal at the LOQ and \% CV of $10 \%$, also at the LOQ. Mean linearity (r2) has been shown for all compounds to be $>0.97$.
Conclusion: This approach by Microflow LC shows valuable advantages minimizing solvent consumption, maximizing LC performance and improving system robustness with minimal sample preparation.

$\overline{\text { P5. La Biométrologie en Santé au Travail, outil de l'évaluation }}$ de la contamination des professionnels de santé par les médicaments anticancéreux

M Canal-Raffin ${ }^{1,2,3}$, Y Goujon ${ }^{4}$, B Martinez ${ }^{5}$, K Khennoufa ${ }^{1,5}$, D Ducint ${ }^{1}$, $\mathrm{K}_{\text {Titier }}{ }^{1}$, C Verdun Esquer ${ }^{6}$, M Molimard ${ }^{1,5}$, P Brochard $^{2,3,6}$

${ }^{1}$ Laboratoire de Pharmacologie Clinique et Toxicologie, Bordeaux ; ${ }^{2}$ ISPED, Laboratoire Santé Travail Environnement, Université V Ségalen, Bordeaux; ${ }^{3}$ Centre INSERM U897-Épidemiologie-Biostatistique, Bordeaux ; ${ }^{4}$ AHI33, Association d'Hygiène Industrielle de Gironde Evaltox, Bordeaux; ${ }_{5}^{5}$ INSERM, U657-Pharmaco-épidémiologie et évaluation de l'impact des produits de santé sur les populations, Bordeaux; ${ }^{6}$ Service de Médecine du Travail, $\mathrm{CHU}$, Bordeaux

Objectif : L'augmentation du nombre de cas de cancers en France ainsi que l'utilisation de plus en plus répandue de médicaments anticancéreux soulèvent la question de l'exposition et de la contamination des professionnels de santé exposés à ces produits, d'autant que certains d'autres eux sont classés cancérogènes par le CIRC. Dans le cadre de l'évaluation du risque chimique chez les travailleurs, et de la réglementation en vigueur incitant les entreprises et les Médecins du Travail à mesurer et surveiller les niveaux d'exposition, le suivi de tout salarié exposé à un agent cancérogène est devenu fortement recommandé en Santé au Travail. Dans ce contexte, grâce à la biométrologie mise en place au sein de notre laboratoire, une campagne de mesures permettant de déterminer les niveaux de contamination au cyclophosphamide des différents salariés de plusieurs établissements de Santé a été menée en Gironde.

Méthodes : Deux professions de santé en contact direct avec les médicaments anticancéreux ont été étudiées : les préparateurs en pharmacie et les infirmières. Le cyclophosphamide a été utilisé comme biomarqueur de l'exposition. Il a été recherché dans les urines des salariés à l'aide d'une méthode par chromatographie liquide couplée à la spectrométrie de masse en tandem (LC/MS-MS). Cette technique validée est très sensible, avec une limite de détection (LD) à $10 \mathrm{pg} / \mathrm{mL}$ et une limite de quantification (LQ) à $20 \mathrm{pg} / \mathrm{mL}$. Les valeurs supérieures à la LQ ont été rendues rapportées à la créatininurie $(\mathrm{ng} / \mathrm{gcu})$. Chaque salarié participant à cette Surveillance Biologique de l'Exposition (SBE) devait fournir 5 prélèvements urinaires répartis autour d'une ou plusieurs journées de manipulation d'au moins une poche de chimiothérapie contenant du cyclophosphamide. En parallèle, des auto-questionnaires ont été recueillis afin de connaître les quantités, les dates, les heures et les modalités de manipulation du cyclophosphamide, en particulier le port des équipements de protection.

Résultats : Au total, 37 agents ont été suivis en 2012: 51,3\% sont des préparateurs en pharmacie issus de 5 Unités de Reconstitution des Chimiothérapies, et 48,7\% sont des infirmières issues de 5 Services de Soins différents. 170 échantillons d'urine ont été recueillis, répartis à $50 \%$ entre les préparateurs en pharmacie et les infirmières. L'analyse de ces prélèvements a révélé dix-huit prélèvements positifs, c'est-à-dire supérieurs à $10 \mathrm{pg} / \mathrm{mL}$. Chez les préparateurs en pharmacie, un seul agent sur les 19 a excrété du cyclophosphamide dans ses urines, dans un seul prélèvement correspondant à la fin de poste et fin de semaine (concentration comprise entre la LD et la LQ, 10-20pg/mL). Chez les infirmières, $27,8 \%$ des agents ont excrété du cyclophosphamide dans leurs urines $(n=5 / 18)$ avec 17 prélèvements sur 85 dépistés positifs : les concentrations étaient comprises entre la LD-LQ pour 3 prélèvements et, les 14 autres prélèvements étaient compris entre 9,5 et 1896 ng/gcu (min-max) avec une valeur médiane de 44,6 ng/gcu. La plupart des infirmières ont au moins 2 prélèvements positifs, permettant ainsi un suivi de la cinétique d'élimination du cyclophosphamide urinaire. 
Conclusion : Le niveau de contamination retrouvé auprès des personnels des Unités de Reconstitution des Chimiothérapies est très faible. Celui dans les Services de Soins chez le personnel infirmier est plus élevé. Cette biométrologie mise en place est un bon outil pour suivre les professionnels en contact avec le cyclophosphamide. Elle intègre toutes les voies d'expositions et évalue le port et l'efficacité des équipements de protection individuelle. Elle permet une traçabilité des expositions pour le dossier médical des salariés. Les résultats de cette campagne de mesures vont permettre de sensibiliser les salariés et d'engager des discussions autour des pratiques professionnelles et des conditions de travail. Des actions de formation vont être menées pour lutter contre ces contaminations. D'autres méthodes analytiques sensibles et spécifiques sont à développer afin de suivre le personnel des Établissements de Santé exposés à des médicaments anticancéreux autres que le cyclophosphamide.

\section{P6. Prévention de la toxicité de l'isoniazide par étude du polymorphisme génétique de la NAT2 chez des sujets sénégalais non fumeurs}

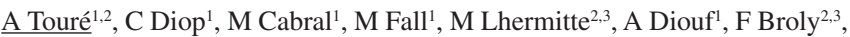
D Allorge ${ }^{2,3}$

${ }^{1}$ Service de Toxicologie-Hydrologie, Faculté de Médecine, Pharmacie et Odontostomatologie, Dakar; Sénégal; ${ }^{2} E A 4483$, UDSL, Université Lille Nord-de-France ; ${ }^{3}$ Service de Toxicologie-Génopathies, CHRU, Lille

Objectif : L'objectif de cette étude était de déterminer le profil génotypique de la NAT2 dans une population sénégalaise et de disposer d'éléments de prévention de la toxicité de l'INH dans la prise en charge de la tuberculose au Sénégal par optimisation du schéma thérapeutique.

Méthodes : Le profil génotypique a été déterminé par PCR-séquençage directe de la région codante du gène de la NAT2. 105 sujets sains non fumeurs ont été sélectionnés et un prélèvement de sang veineux sur tube avec EDTA a été effectué chez tous les sujets. L'ADN génomique, obtenu après extraction a été stocké à $-20^{\circ}$ jusqu' au moment des manipulations.

Résultats : Onze variants alléliques de la NAT2 ont été identifiés chez les sujets sénégalais testés. Les 4 allèles les plus fréquents sont $N A T 2 * 5$ $(35,7 \%), N A T 2 * 6-(21,0 \%), N A T 2 * 12-(16,7 \%)$ et NAT2*14- $(10,0 \%)$. D'autres allèles, tels que l'allèle sauvage $(N A T 2 * 4)$, ont été retrouvés à des fréquences inférieures à $10 \%$. Pour le profil génotypique, 48,6\% sont prédits acétyleurs rapides et $47,6 \%$ acétyleurs lents. Cependant, 4 sujets ( $3,8 \%)$ ont été considérés de phénotype inconnu (non classés) car possédant un allèle d'effet fonctionnel sur la NAT2 inconnu.

Conclusion : Ce travail présente les premiers résultats de l'étude de la NAT2 dans la population sénégalaise, et les résultats pourront être utilisés pour une meilleur optimisation de l'utilisation de l'INH dans le prise en charge efficiente de la tuberculose, maladie à forte prévalence en Afrique.

\section{$\overline{\text { P7. Développement d'outils analytiques pour une application }}$ en routine en Santé au Travail dans le cadre d'une} Surveillance Biologique de l'Exposition professionnelle aux médicaments anticancéreux

$\underline{\text { K Khennoufa }^{1,3}, \text { C Verdun-Esquer }}{ }^{2}$, B Martinez ${ }^{3}$, D Ducint ${ }^{1}$, K Titier ${ }^{1}$, M Molimard ${ }^{1,3}$, P Brochard $^{2,4,5}$ M Canal-Raffin ${ }^{1,4,5}$

${ }^{1}$ Laboratoire de Pharmacologie Clinique et Toxicologie, CHU, Bordeaux ; ${ }^{2}$ Service de Médecine du Travail, CHU, Bordeaux; ${ }^{3}$ INSERM, U657, Bordeaux ; ${ }^{4}$ INSERM, ISPED, U897, Bordeaux ; ${ }^{5}$ ISPED, Laboratoire Santé Travail Environnement, Université V Ségalen, Bordeaux

Objectif: L'administration de plus en plus répandue de médicaments anticancéreux par les professionnels de santé soulève la question de leur contamination par ces produits, d'autant que certains d'autres eux sont classés cancérogènes par le CIRC. Différentes méthodes d'analyse pour rechercher et/ ou doser certains médicaments anticancéreux dans les urines de professionnels exposés ont déjà été publiées. Pour une exposition au cyclophosphamide, à l'ifosfamide ou au méthotrexate, les méthodes les plus sensibles ont une limite de détection (LD) de $10 \mathrm{pg} / \mathrm{mL}, 20 \mathrm{pg} / \mathrm{mL}$ et $200 \mathrm{pg} / \mathrm{mL}$ respectivement. L'objectif de ce travail a été de développer des outils analytiques pour une application en routine en Santé au Travail et, d'améliorer les limites de détection pour ces trois molécules. Ces outils ont ensuite été appliqués dans le cadre d'une étude pilote de Surveillance Biologique de l'Exposition (SBE) professionnelle à ces trois médicaments anticancéreux.

Méthodes : Deux méthodes de dosage ont été développées et validées par chromatographie liquide à haute performance couplée à la spectrométrie de masse en tandem (CLHP-MS/MS, quattromicro, Waters ${ }^{\circledR}$ ). La colonne analytique est une colonne $\mathrm{dC}_{18}\left(100 \times 2,1 \mathrm{~mm} ; 5 \mu \mathrm{m}\right.$, Atlantis, Waters $\left.{ }^{\circledast}\right)$ et la phase mobile se compose d'un mélange de tampon de formiate d'ammonium $(4 \mathrm{mM}$, $\mathrm{pH}=3,2$ ) et d'acétonitrile. La première méthode permet le dosage simultané du cyclophosphamide et de l'ifosfamide dans les urines après une extraction liquideliquide. La deuxième méthode, quant à elle, dose le méthotrexate urinaire après une extraction solide-liquide. Ces méthodes ont été utilisées dans le cadre d'une étude pilote non-interventionnelle prospective de SBE professionnelle à ces trois médicaments. Elle a été menée dans deux Établissements de Santé en Gironde. Chaque participant a fourni des prélèvements urinaires durant une semaine de travail et a rempli un auto-questionnaire afin de connaître les quantités, les dates, les heures et les modalités de manipulation de ces trois médicaments, en particulier le port des équipements de protection. Tout prélèvement supérieur à la LD a été considéré comme positif.

Résultats : Les méthodes validées pour les trois molécules présentent une bonne linéarité entre 20 et $500 \mathrm{pg} / \mathrm{mL}\left(\mathrm{r}^{2}>0,99\right)$. Les limites de détection sont de $10 \mathrm{pg} / \mathrm{mL}$ et les limites de quantification de $20 \mathrm{pg} / \mathrm{mL}$ pour les trois médicaments. Au cours de notre étude, deux Unités de Reconstitution des Chimiothérapies et deux Services de Soins ont été suivis en 2012. Au total, 38 agents ont participé dont 13 préparateurs en pharmacie, 10 infirmières, 4 ASH, 3 aide-soignantes et 8 autres personnels. Trois cent cinquante sept prélèvements urinaires ont été recueillis. Dans les Unités de Reconstitution des Chimiothérapies, tous les prélèvements sont inférieurs à 10 pg/mL (LD) pour les 3 molécules. Aucun agent n'a donc été contaminé. Dans les Services de Soins, 2 personnes sur 16 ont été contaminées (12,5\%); les niveaux de concentrations urinaires atteints étaient tous supérieures à la LQ. Il s'agit d'une infirmière avec 2 prélèvements positifs en ifosfamide $(25-37 \mathrm{pg} / \mathrm{mL})$ et, d'une ASH avec 3 prélèvements positifs en méthotrexate (26,6-57,1 pg/mL). Conclusion: Les outils analytiques (CLHP-MS/MS) que nous avons développés sont spécifiques. Concernant, le cyclophosphamide, la sensibilité de la méthode est aussi performante que celle déjà publiée. Pour l'ifosfamide et le méthotrexate, ces outils ont permis d'améliorer les limites de détection et de quantification par rapport aux données de la littérature. Dans le cadre de notre étude pilote, les niveaux de concentrations retrouvés dans les urines des personnels contaminés sont très faibles. Pour le méthotrexate, ils sont inférieurs aux LD publiées à ce jour. Ainsi, grâce à cette nouvelle limite de détection pour le méthotrexate, trois prélèvements ont été détectés positifs, permettant d'éviter le rendu de faux négatifs. L'utilisation des outils analytiques aussi performants en termes de sensibilité et de spécificité est indispensable pour le dépistage et le suivi en routine du personnel exposé aux médicaments anticancéreux dans le cadre de la surveillance biologique de l'exposition professionnelle à ces médicaments en Santé au Travail.

\section{P8. Apport de la LC-MS/MS haute résolution pour le screening toxicologique dans le sang total}

T Gicquel $^{1}$, S Lepage ${ }^{1}$, B Duretz ${ }^{2}$, I Morel ${ }^{1}$

${ }^{1}$ Laboratoire de toxicologie biologique et médicolégale, CHU Pontchaillou, Rennes ; ${ }^{2}$ Thermo Fisher Scientific, Les Ulis

Objectif : Le screening toxicologique nécessite l'identification d'un nombre illimité de composés dans des matrices souvent complexes. De plus, cette identification doit se faire dans certains cas de manière rétrospective pour 
l'identification de nouvelles substances. Le couplage d'un quadrupôle avec la technologie Orbitrap (Q Exactive ${ }^{\circ}$, Thermo Fisher Scientific) été évaluée pour le screening toxicologique sur des échantillons de sang total issus de demandes cliniques ou d'autopsie.

Méthodes : Après ajout d'un étalon interne (flurazepam), l'échantillon sanguin $(1 \mathrm{~mL})$ est extrait au moyen d'un toxitube $\mathrm{A}^{\circledR}$ (Varian). La phase organique est ensuite évaporée et reprise par $500 \mu \mathrm{L}$ de phase mobile, l'échantillon est analysé par un couplage chromatographie liquide/ spectromètre de masse de type Q Exactive ${ }^{\circledR}$. L'ionisation se fait en mode electrospray (H-ESI), la séparation chromatographique de 30 minutes est réalisée sur une colonne Gold PFP $(150 \times 2,1 \mathrm{~mm}, 5 \mu \mathrm{m})$. Les données sont collectées en mode MS et MS/MS par ionisation positive et négative au cours d'une même injection. Les résolutions sont respectivement de $70 \mathrm{~K}$ et de $17,5 \mathrm{~K}$ (FWHM) pour les modes full scan et MS².

Résultats : L'identification des molécules est basée sur la masse exacte de l'ion parent (précision de $5 \mathrm{ppm}$ ), le temps de rétention de l'analyte, le profil isotopique et la masse exacte de plusieurs fragments caractéristiques (précision de $5 \mathrm{ppm}$ ). Toutes les données ont été traitées avec le logiciel ExactFinder $^{\circledR}$. Les résultats obtenus à partir de 50 échantillons sanguins ont été corrélés à ceux obtenus lors d'un screening classique par chromatographie liquide couplée à une barrette de diode (UPLC-Acquity ${ }^{\circledR}$, Waters). L'ensemble des molécules identifiées par la détection en barrette de diodes a pu être retrouvé, après une dilution au cinquième de l'échantillon, par mesure de la masse exacte. De plus, les données étant acquises en mode full scan, il a été possible de détecter des molécules supplémentaires, non incluses dans la base de données du fournisseur, via la consultation de banques de données sur Internet.

Conclusion : La comparaison des deux méthodes a permis de confirmer l'intérêt d'utiliser la technologie de masse exacte pour le screening toxicologique du fait de sa grande sensibilité et spécificité. L'avantage supplémentaire de cette approche est qu'elle permet des études rétrospectives à partir de banques de données de molécules, telles que celles récemment apparues sur le marché des substances illicites.

\section{P9. Mise au point d'une méthode d'extraction en ligne couplée à la LC-MS/MS pour la quantification du THC et de ses principaux métabolites sur sang total}

\section{$\underline{\text { V Thibert }}^{1}$, B Duretz ${ }^{1}$, C Petit ${ }^{2}$, M Lachambre $^{2}$}

${ }^{1}$ Thermo Fisher Scientific, Courtabouf, Les Ulis; ${ }^{2}$ Laboratoire Analysis Expertise, Epinal

Objectif : L'objectif de ce travail était de développer une méthode simple et rapide pour l'analyse du $\Delta^{9}$-tétrahydrocannabinol (THC), et de ses deux métabolites, le 11-hydroxy- $\Delta^{9}$-tetrahydrocannabinol (OH-THC) et l'acide 11-nor- $\Delta^{9}$-tetrahydrocannabinol-9-carboxylique (THC-COOH), sur sang total en utilisant la chromatographie liquide TurboFlow couplée à la spectrométrie de masse.

Méthodes : L'extraction en ligne du THC, de l'OH-THC et du THC-COOH a été optimisée pour leur analyse dans du sang après une précipitation de protéines par de l'acétonitrile acidifié. $90 \mu \mathrm{L}$ du surnageant ont été extraits en ligne par une colonne TurboFlow Cyclone $\mathrm{P}(0,5 \times 50 \mathrm{~mm})$, puis une étape de séparation a été effectuée sur une colonne Accucore C18 $(50 \times 2,1 \mathrm{~mm} \times 2,6 \mu \mathrm{m})$, suivie d'une analyse par spectrométrie de masse en tandem. L'intégralité des étapes d'extraction en ligne et de séparation chromatographique est réalisée en 11 minutes.

Résultats : Des courbes de calibration avec l'utilisation de standards internes deutérés ont été obtenues pour les trois molécules pour des concentrations allant de $0,5 \mathrm{ng} / \mathrm{mL}$ à $100 \mathrm{ng} / \mathrm{mL}$ avec une relation linéraire $\left(\mathrm{r}^{2}>0,99\right)$. Les limites de quantification obtenues pour les trois molécules sont de $0,5 \mathrm{ng} / \mathrm{mL}$, ce qui est conforme aux objectifs analytiques du laboratoire dans le cadre des missions d'expertises toxicologiques. La corrélation avec la méthode GC-MS du laboratoire a été vérifiée sur 50 échantillons.
Conclusions : La méthode développée permet la quantification du THC et ses deux métabolites principaux par une extraction automatisée basée sur de la chromatographie par flux turbulent, avec une bonne sensibilité. Le temps d'analyse est finalement réduit par rapport à une méthode GC-MS et conserve en même temps de bonnes performances.

\section{P10. Bilan des intoxications graves du service de réanimation médicale au CHU de Nantes en 2012 : circonstances de demande de criblage toxicologique et de dosages spécifiques}

G Deslandes ${ }^{1}$, R Bouquie$^{1}$, H Grison-Hernando ${ }^{1}, \mathrm{C}$ Bruneau $^{2}, \mathrm{C}$ Renaud ${ }^{1}$, E Dailly ${ }^{1}$, C Azoulay ${ }^{1}$, C Monteil-Ganiere ${ }^{1}$, A Pineau ${ }^{1}$, P Harry ${ }^{2}$, D Villers ${ }^{3}$, P Jolliet $^{1}$

${ }^{1}$ Laboratoire de Pharmacologie-Toxicologie, CHU, Nantes; ${ }^{2}$ Centre Antipoison-Toxicovigilance, $\mathrm{CHU}$, Angers; ${ }^{3}$ Service de Réanimation Médicale, $\mathrm{CHU}$, Nantes

Objectif : Synthèse et analyse des cas graves d'intoxication aigües en 2012, dans un service de réanimation médicale en CHU.

Méthodes : Sur les 893 admissions en réanimation médicale au CHU de Nantes en 2012, ont été extraits tous les dossiers pour lesquels le code diagnostic principal est une intoxication. Sur les dossiers retenus, les informations suivantes ont été relevées à partir du dossier du patient : âge, sexe, intoxication volontaire ou accidentelle, évolution, médicaments suspectés, durée d'hospitalisation en réanimation, intubations, dosages d'alcoolémie, de la paracétamolémie, criblage toxicologique, notifications au Centre Régional de Pharmacovigilance (CRPV) et appels au Centre Antipoison et de Toxicovigilance (CAP-TV).

Résultats : Sur les 893 admissions en 2012, 113 (12,7\%) étaient des intoxications dont $94 \%$ volontaires. Sur ces 113 admissions, $57 \%$ des patients sont des femmes; l'âge moyen est de 44 ans (17 à 85). La durée moyenne du séjour est de 3,2 jours ( 0 à 18$)$. Les médicaments suspectés sont connus dans $85 \%$ des cas (aveux du patient, blisters retrouvés dans les poubelles, aux pieds du patient, par les pompiers, la famille ou le SMUR...). 1 à 9 médicaments sont supposés être ingérés par patient (moyenne 2,46) et sont répartis de la manière suivante : $34 \%$ de benzodiazépines et apparentés (bromazépam $8 \%$, oxazépam $7 \%$, zolpidem $5,6 \%$, zopiclone $2,6 \%$ ), $19 \%$ de neuroleptiques (cyamémazine $11 \%$ ), $19 \%$ d'antidépresseurs (citalopram 5\%, venlafaxine 3,5\%), $6 \%$ d'anticonvulsivants, (acide valproïque $2,6 \%$, lamotrigine $1,3 \%$ ), $3 \%$ de paracétamol, $3 \%$ d'opiacés, $3 \%$ d'antihistaminique $\mathrm{H} 1,6 \%$ de cardiotropes (bétabloquants $3 \%$ ) et autres: $7 \%$ (potassium, lithium, insuline, œstradiol, baclofène...). Deux notifications seulement ont été effectuées au CRPV, le CAP-TV a été consulté 14 fois. Au niveau analytique, le criblage toxicologique a été effectué dans $27 \%$ des cas; le dosage du paracétamol dans $40 \%$ des cas (dont une intoxication massive) ; le dosage d'éthanolémie a été réalisé dans $82 \%$ des cas et était positif chez 38 patients $(33 \%)$ avec une moyenne de $1,9 \mathrm{~g} / \mathrm{L}(0,7$ à $3,6 \mathrm{~g} / \mathrm{L})$. Au niveau clinique, $77 \%$ des patients ont été intubés et ventilés au cours de leur séjour. Deux décès sont survenus dont une intoxication massive à l'amisulpride, et une pendaison associée à une intoxication au prazépam). À l'issue de leur séjour en réanimation, $53 \%$ des patients ont été transférés dans d'autres services (24\% en unité d'hospitalisation de courte durée, $22 \%$ en psychiatrie, $7 \%$ dans des services de médecine (néphrologie, pneumologie, cardiologie...)) et $45 \%$ des patients sont retournés directement à leur domicile.

Conclusion : Le criblage toxicologique est réalisé sur demande du service avec 2 indications principales: quand les signes cliniques ne coïncident pas avec le toxidrome et quand les toxiques ne sont pas connus. Le dosage spécifique de molécules est systématiquement réalisé quand il existe une corrélation entre gravité de l'intoxication et concentration sanguine. À noter qu'aucune intoxication au méprobamate n'a été répertoriée en 2012 (16 en 2008, 7 en 2009, 3 en 2010, 4 en 2011); ce principe actif seul ou associé a été retiré du marché le 10/01/2012 par l'ANSM. 
P11. Intérêt de la spectrométrie de masse hybride « quadripôle-temps de vol » pour l'étude du métabolisme des xénobiotiques : à propos de la méthoxyisoflavone

Y Lecompte $^{1,2}$, C Richeval $^{3}$, L Humbert ${ }^{3}$, M Perrin ${ }^{4}$, P Arpino ${ }^{5}$

${ }^{1}$ Laboratoire de contrôle radiotoxicologique, Service de protection radiologique des Armées, Clamart; ${ }^{2}$ Département Toxicologie, Institut de Recherche Criminelle de la Gendarmerie nationale, Rosny-SousBois ; ${ }^{3}$ Laboratoire de Toxicologie et de Génopathies, Centre de Biologie Pathologie, CHRU, Lille ; ${ }^{4}$ tat major de la Marine, bureau « Maîtrise des risques », Paris ; ${ }^{5}$ Laboratoire d'électrochimie, chimie des interfaces et modélisation pour l'énergie, Chimie-Paristech, Paris

Objectif : La méthoxyisoflavone (5-méthyl-7-méthoxyisoflanone) est une isoflavone de synthèse employée pour ses propriétés anabolisantes par les culturistes. L'implication des métabolites de cette molécule dans des réactions croisées avec les anticorps dirigés contre le 11-nor-9-carboxy$\Delta$ 9-tétrahydrocannabinol (THC-COOH) des tests de dépistage urinaire des cannabinoïdes a été précédemment démontrée. Dans cette étude, les métabolites urinaires de la méthoxyisoflavone sont recherchés par chromatographie liquide haute performance couplée à un spectromètre de masse hybride quadripôle-temps de vol (UPLC-Q-TOF).

Méthodes : Les échantillons d'urine proviennent de trois volontaires sains inclus dans un essai d'administration de la méthoxyisoflavone. Après hydrolyse enzymatique et extraction liquide-liquide, ces échantillons sont analysés par UPLC-Q-TOF (Waters, XEVO-G2-Q-TOF®) à l'aide d'une source d'ionisation par électronébulisation (ESI) en mode positif. Les spectres de masse sont acquis en mode $\mathrm{MS}^{\mathrm{E}}$ avec le logiciel MassLynx ${ }^{\circledR}$ et analysés avec les logiciels MetaboLynx ${ }^{\circledast}$ et MassFragment ${ }^{\circledR}$ (Waters, Milford, USA). L'exploitation des données est complétée à l'aide du logiciel ACD/Spectrus ${ }^{\circledR}$ - version bêta (ACD/Labs, Toronto, Ontario, Canada).

Résultats : Deux voies de métabolisation et cinq métabolites de la méthoxyisoflavone ont été identifiés, selon que la mono- et la dihydroxylation du cycle B sont précédées ou non d'une O-déméthylation. Les spectres de masse $\mathrm{MS}^{\mathrm{E}}$ de la méthoxyisoflavone et des métabolites détectés traduisent la perte de fragments neutres $\mathrm{CO}$ et $\mathrm{H}_{2} \mathrm{O}$ et, pour les molécules méthoxylées, du radical $\mathrm{CH}_{3}{ }^{*}$ caractéristiques des flavonoïdes. Une double perte de $\mathrm{CO}$ et une fragmentation de type Rétro-Diels-Alder selon les liaisons 1 et 3, spécifiques des isoflavones, sont systématiquement observées.

Conclusion : De par sa sensibilité et la mesure des masses exactes, l'UPLCQ-TOF, employée en mode $\mathrm{MS}^{\mathrm{E}}$ avec un logiciel de déconvolution dédié à la recherche des métabolites, constitue un outil puissant pour la détermination du métabolisme de xénobiotiques inconnus telles que la méthoxyisoflavone, à partir d'échantillons d'urine et en l'absence de molécules de référence.

\section{P12.Évaluation de l'exposition au benzène et au 1,3-butadiène liée au trafic automobile}

M Borgie ${ }^{1}$, A Garat ${ }^{2}$, F Cazier ${ }^{3}$, A Delbende ${ }^{3}$, C Richeval ${ }^{2}$, D Allorge ${ }^{2}$, D Courcot $^{1}$, P Shirali ${ }^{1}$, Z Dagher $^{4}$

${ }^{1}$ Unité de chimie environnementale et interactions sur le vivant, Université du Littoral-Côte d'Opale, Dunkerque ; ${ }^{2} U F$ de toxicologie, pôle de biologie pathologie génétique, CHRU de Lille; ${ }^{3}$ Centre commun de mesures, Université du Littoral-Côte d'Opale, Dunkerque ; ${ }^{4}$ Groupe de recherche en molécules bioactives, Université Libanaise, Beyrouth

Objectif : Le benzène et le 1,3-butadiène (BD) ont été classés comme cancérogènes pour l'homme selon le CIRC compte tenu des effets génotoxiques et épigénotoxiques observés lors des études in vitro/in vivo et de bio-surveillance. Nous nous sommes intéressés à caractériser l'exposition à ces émissions liées au trafic automobile dans un milieu professionnel.

Méthodes : Nous avons entrepris une campagne dans la ville de Beyrouth auprès de 24 policiers travaillant comme agents de la circulation et 23 policiers travaillant dans les bureaux. Une surveillance de l'exposition individuelle aux benzène, toluène, éthylbenzène et xylène (BTEX) a été réalisée par des badges de diffusion passive, GABIE (Gas Adsorbent Badge for Individual Exposure) (Arelco, France). Des prélèvements urinaires ont été réalisés, début de travail et en fin de poste, pour mesurer les biomarqueurs d'exposition au benzène (acides trans, trans-muconique (t,t-MA) et phényle mercapturique (PMA) urinaires) et au 1,3-butadiène (acides monohydroxybutényle mercapturique (MHBMA) et dihydroxybutyle mercapturique (DHBMA) urinaires). La quantification des BTEX a été réalisée par GC-MS/MS, celle du t,t-MA par HPLC/UV et celle du S-PMA, du MHBMA et du DHBMA par UPLC/ESI(-)MS/MS en mode MRM. Résultats : Nous avons constaté que le niveau d'exposition individuelle au benzène des agents de la circulation était plus élevé que celle publiée dans d'autres travaux (Maffei et al., Mutat Res. 2005 ; Pilidis et al., Environ Monit Assess. 2008 ; Rossnerova et al., Mutat Res. 2009 ; Arayasiri et al., Sci Total Environ. 2010). Le niveau du t,t-MA est significativement différent entre les agents de la circulation et le groupe contrôle et est significativement corrélé avec les niveaux d'exposition personnelle aux composés BTEX. Cependant, les niveaux du MHBMA ne montraient pas de variation significative. Alternativement, la concentration du DHBMA était significativement plus élevée chez les agents de la circulation versus le groupe contrôle et est significativement corrélé avec les niveaux d'exposition personnelle aux composés BTEX. De plus, il n'y avait aucune influence du tabagisme sur les niveaux du DHBMA.

Conclusion : Ces résultats suggèrent que le DHBMA est plus adapté que le MHBMA comme biomarqueur d'exposition au 1,3-butadiène chez l'homme. Les agents de la circulation, qui sont exposés au benzène et au 1,3-butadiène émis du trafic automobile de la ville de Beyrouth, parcourent plus le risque du développement de pathologies, tel que le cancer, que les policiers travaillant dans des bureaux.

\section{P13. Variations d'un Indice Biologique d'Exposition au chrome en présence d'un dispositif médical}

C Sayag $^{1}$, A Nicolas ${ }^{2}$, F Pelissier ${ }^{1}$, JL Galibert ${ }^{3}$, N Franchitto ${ }^{1}$

${ }^{I}$ Centre Antipoison et de Toxicovigilance, Centre Hospitalier Universitaire, Toulouse; ${ }^{2}$ Toxilabo, Laboratoire de Toxicologie et bio-toxicologie professionnelles, Nantes ; ${ }^{3}$ Médecine du Travail, Figeac

Objectif : En médecine du travail, la surveillance de l'exposition au chrome utilise l'Indice Biologique d'Exposition (IBE) ou Chrome urinaire qui est un bon indicateur de l'exposition récente et chronique à toutes les formes de chrome, et dont la valeur guide de référence en milieu de travail est de $0,52 \mu \mathrm{g} / \mathrm{g}$ de créatinine. Un salarié, travaillant dans une entreprise orientée dans la fabrication de pales et d'hélices, a présenté un IBE élevé dans les suites de la pose de deux prothèses de hanche à 7 mois d'intervalle, prothèse de resurfaçage métal contre métal contenant chrome, cobalt, molybdène et nickel, qui pourraient être responsables, selon les données de la littérature, d'un relargage de chrome.

Méthodes : Des prélèvements ont été réalisés chaque année (en fin de poste et fin de semaine) et au retour du salarié à la suite de chaque intervention chirurgicale. La technique utilisée est la spectrométrie d'absorption atomique électrothermique (SAA éléctrothermie).

Résultats : Le niveau d'exposition en chrome atmosphérique des salariés était normal, notamment au sein d'un Groupe Homogène d'Exposition (GHE) constitué du salarié et de deux autres de ses collègues. Les résultats de la surveillance IBE du chrome urinaire de ce salarié restaient inferieurs à la valeur de référence les deux années avant la pose de la première prothèse, pour atteindre une valeur de $1,2 \mu \mathrm{g} / \mathrm{g}$ de créatinine à trois mois de l'intervention chirurgicale, et de $2 \mu \mathrm{g} / \mathrm{g}$ de créatinine à cinq mois. Après la pose de la seconde prothèse, les résultats étaient respectivement de $10,2 \mu \mathrm{g} / \mathrm{g}$ de créatinine à trois mois puis de $14,9 \mu \mathrm{g} / \mathrm{g}$ de créatinine à cinq mois.

Conclusion: La normalité des dosages atmosphériques et urinaires de chrome des autres salariés du groupe, et les dosages élevés secondaires à la pose successive des 2 prothèses chez ce salarié amènent l'hypothèse d'une exposition au chrome par relargage de ce métal par le matériel prothétique 
mis en place. Ceci rappelle la nécessité de vigilance de la part des équipes médicales dans des situations similaires.

\section{P14. Point sur une nouvelle drogue de synthèse : le 5-IT}

D Debruyne ${ }^{1,2}$, R Le Boisselier ${ }^{2}$, M Loilier ${ }^{1}$, A Coquerel ${ }^{1,2}$

${ }^{1}$ Laboratoire de Pharmacologie-Toxicologie, CHU de Caen; ${ }^{2}$ Centre d'Évaluation et d'Information suer la Pharmacodépendance-Addictovigilance, CHU de Caen

Introduction: Le 5-IT ou 5-(2-aminopropyl)indole est une drogue de synthèse qui circule depuis 2012 dans divers États Membres de la Communauté Européenne. En septembre 2012, les deux organisations EMCDDA (European Monitoring Center for Drugs and Drug Addiction) et Europol (European Police Office) ont conclu qu'elles avaient suffisamment de données pour produire un rapport commun sur le 5-IT.

Méthodes : À partir du rapport conjoint EMCDDA/Europol, de données de la littérature, de consultations de forum d'usagers et du réseau des Centres d'addictovigilance (CEIP-A), nous faisons un bilan des connaissances actuelles sur le 5-IT.

Résultats: Le 5-IT est une drogue de synthèse associant une chaine propylamine à un noyau indole; c'est une structure intermédiaire entre une phenylethylamine et une tryptamine ; c'est un isomère de position d'une autre drogue de synthèse, le 3-IT ou AMP ou $\alpha \mathrm{MT}$ (méthyltryptamine). Le 5-IT circule dans la communauté européenne puisqu'il a fait l'objet de saisies par les services de douane et de police, surtout depuis 2012, dans 7 pays européens ; aucun élément ne permet de dire s'il circule sur le territoire national. Le 5-IT est vendu sous diverses présentations (sachets de poudre, comprimés, gélules) et a été retrouvé dans des poudres vendues sous l'appellation «benzo fury ». Les caractéristiques analytiques du 5-IT sont connues (spectre RMN, spectre UV, spectres de masse) mais il est difficile à différencier de l' $\alpha$ MT. C'est un produit d'accès facile sur Internet, en majorité sur des sites hébergés par le Royaume Uni. Il est considéré comme « légal », et d'un prix comparable à celui de l' $\alpha$ MT, de l'ordre de $45 €$ le gramme. Il n'y a pas d'études expérimentales, publiées, spécifiques au 5-IT mais des effets sont décrits par les usagers. C'est une substance qui présente des propriétés stimulantes, entactogènes et psychodysleptiques globalement comparables à celles des autres stimulants de la classe des cathinones, amphétamines, cocaïne. Le produit, administré par voie orale, nasale voire IV, est considéré comme puissant, agissant lentement (risque de ré- administration rapide) et longtemps. Des effets indésirables nombreux, eux aussi caractéristiques de la classe des stimulants ont été rapportés et parmi eux, les effets cardio- vasculaires sont les plus dangereux. Plusieurs cas médicolégaux, confirmés par des analyses toxicologiques, ont imputé le décès au 5-IT et ont fait l'objet d'une vaste communication à l'égard des usagers de drogue. D'autres cas de décès associant le 5-IT à d'autres substances ont également été rapportés. Suite aux alertes effectuées sur le Net, ce produit semble apparaître, aux yeux des usagers, comme risqué et peu attrayant. Ce produit est non connu, en France, comme substance active ou intervenant dans la synthèse d'une spécialité pharmaceutique Il ne figure, en France, ni sur la liste des substances vénéneuses, ni des stupéfiants et psychotropes, ni des précurseurs.

Conclusion : Aucun signalement n'a été transmis par le réseau des CEIP-A et l'OFDT, et il ne semble pas qu'il y ait, pour l'instant, un usage notoire de ce produit sur le territoire national.

\section{$\overline{\mathrm{P} 15 . \mathrm{H}_{2} \mathrm{~S} \text { : Intoxication accidentelle mortelle d'une fillette à }}$ domicile}

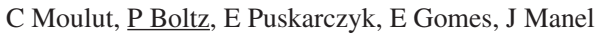

Centre Antipoison et de Toxicovigilance, $\mathrm{CHU}$, Nancy

Objectif : Produit lors de la décomposition de matières organiques soufrées, l'hydrogène sulfuré ( $\mathrm{H}_{2} \mathrm{~S}$, CAS 7783-06-4) est un gaz incolore, d'odeur fétide caractéristique «d'œuf pourri ». Les intoxications au domicile sont exceptionnelles. Le cas du décès d'une fillette de six ans intoxiquée à son domicile est rapporté. La prise en charge toxicologique ainsi que la prévention de tels accidents sont discutés.

Méthodes : Description du cas et des circonstances d'exposition. Synthèse bibliographique sur la toxicité de l'hydrogène sulfuré, la prise en charge et la prévention des intoxications par $\mathrm{H}_{2} \mathrm{~S}$.

Résultats : $\mathrm{H}_{2} \mathrm{~S}$ est toxique par inhalation, il associe un effet irritant topique et une toxicité systémique comparable à celle de l'ion cyanure avec notamment l'inhibition de la respiration mitochondriale cellulaire. Le système nerveux central et l'appareil cardiovasculaire en sont les principales cibles. L'importance de l'exposition est favorisée par un effet lésionnel local (touchant de manière prolongée les terminaisons olfactives), associé à une diffusion rapide du gaz. Dès $500 \mathrm{ppm}$ en air ambiant, une perte de connaissance soudaine liée à des troubles neurologiques (coma, crises convulsives...) et cardiaques sévères (modifications du rythme cardiaque et de la tension artérielle) puis respiratoires (œdème pulmonaire) peuvent entrainer le décès. L'atteinte systémique primitivement fonctionnelle peut s'accompagner de séquelles (induction apoptotique et syndrome post intervallaire). Dans le cas décrit, la fillette chute inanimée dans la salle de bain; la mère est alors alertée mais elle est limitée dans son intervention par des troubles visuels et des céphalées. Deux autres personnes sur les lieux, ainsi qu'un voisin se plaignent de céphalées, d'irritations oculaires et oropharyngées ; les oiseaux domestiques seront retrouvés morts dans leur cage. L'odeur pestilentielle motive l'aération large et précoce des lieux. Sur place, l'équipe du SMUR retrouve l'enfant inconscient avec un pouls fémoral faible. Un arrêt cardiaque survient quelques instants plus tard. La reprise d'une activité cardiaque spontanée efficace est obtenue après $2 \mathrm{~min}$ de réanimation $\left(\mathrm{TA} 80 / 44 \mathrm{mmHg}, \mathrm{FC}=133 / \mathrm{min}, \mathrm{SpO}_{2}=100 \%\right.$, $\mathrm{PetCO}_{2}=64 \mathrm{mmHg}$ ); une expectoration rose mousseuse est retrouvée à l'aspiration. Les circonstances, l'odeur caractéristique, l'aspect collectif et le toxidrome observé permettent d'évoquer l'hypothèse d'une intoxication par $\mathrm{H}_{2} \mathrm{~S}$. La présence d' $\mathrm{H}_{2} \mathrm{~S}$ est confirmée tardivement par une détection atmosphérique. La prise en charge toxicologique classique d'une intoxication grave par $\mathrm{H}_{2} \mathrm{~S}$ est alors entreprise : oxygénothérapie normobare puis hyperbare. Devant l'évolution péjorative, l'intérêt de l'administration d'hydroxocobalamine et $\mathrm{N}$-acétylcystéine est discuté. Malgré deux épisodes d'arrêts cardiorespiratoires résolutifs, la fillette décède probablement des conséquences de l'anoxie cérébrale (pas d'autopsie réalisée, prélèvements conservatoires non valorisés). L'utilisation d'un déboucheur acide sur un amas de matière organique d'une colonne d'évacuation de l'immeuble d'habitation semble être à l'origine d'un dégagement important $\mathrm{d}^{\prime} \mathrm{H}_{2} \mathrm{~S}$ dans les pièces de vie.

Conclusion : Ce cas démontre la possibilité d'une intoxication accidentelle fatale au domicile par exposition à $\mathrm{H}_{2} \mathrm{~S}$. Contrairement aux déboucheurs alcalins, les déboucheurs acides sont susceptibles de le produire chimiquement. La restriction d'usage, sinon l'interdiction de déboucheurs acides pourrait constituer une mesure appropriée de gestion du risque.

P16. Quantification de la cerbérine, la nériifoline (déacétylcerbérine), la tanghinine et la déacétyltanghinine par CLUHP-BPD/SM dans les graines du Cerbera manghas L. Confirmation par CLUHP-SM² haute résolution

Y Gaillard ${ }^{1}$, J Carlier $^{1}$, J Guitton ${ }^{2}$, F Bevalot ${ }^{3}$, L Fanton $^{4}$

${ }^{1}$ Laboratoire LAT-LUMTOX, La Voulte-sur-Rhône; ${ }^{2}$ Laboratoire de toxicologie, Faculté de pharmacie, Lyon; ${ }^{3}$ Laboratoire LAT-LUMTOX, Lyon ; ${ }^{4}$ Institut de Médecine Légale, Faculté de médecine, Lyon

Objectif : La toxicité du faux manguier (C. manghas L.) est notoire. Sa graine fut activement employée comme poison d'ordalie et la plante compte aujourd'hui parmi les plus mortelles du littoral asiatique méridional. L'ingestion d'une seule de ces graines peut être létale.

Les cardénolides glycosidiques sont responsables de la cardiotoxicité des arbres du genre Cerbera. Nous avons identifié et déterminé la concentration 
des principaux cardénolides présents dans des graines du faux manguier originaires de Thaillande.

Méthodes : Les analyses sont réalisées en chromatographie en phase liquide à ultra-haute performance couplée à une détection par barrette de photodiode et spectrométrie de masse (CLUHP-BD/SM). La nériifoline est dosée dans le fruit à $219 \mathrm{~nm}$ par la méthode des ajouts dosés. La quantification de la cerbérine, la tanghinine et la déacétyltanghinine est le résultat d'une approximation par analogie structurale avec la nériifoline, seul étalon commercialement disponible (spectre UV-visible identique). L'identification des composés est confirmée par leur spectre de fragmentation et leur masse exacte (CLUHP-SM ${ }^{2}$ haute résolution) (nériifoline : $m / z$ 535,3259 $\pm 5 \mathrm{ppm}$, cerbérine: $m / z$ 577,3375 $\pm 5 \mathrm{ppm}$, tanghinine: $m / z$ 591,3169 $\pm 5 \mathrm{ppm}$, déacétyltanghinine : $m / z$ 549,3055 $\pm 5 \mathrm{ppm})$.

Résultats : La cerbérine, la nériifoline (déacétylcerbérine), la tanghinine et la déacétyltanghinine représentent la quasi-totalité des hétérosides cardiotoxiques présents dans la graine du faux manguier. La graine du fruit séché présentait des concentrations de 285,$9 ; 804,2 ; 621,4$ et $1209,1 \mu \mathrm{g} . \mathrm{g}^{-1}$ respectivement. La graine du fruit frais présentait des concentrations de 2,3; 47,$0 ; 3,5$ et $49,4 \mu \mathrm{g} \cdot \mathrm{g}^{-1}$.

Conclusion : Les concentrations mesurées en nériifoline et en cerbérine dans la graine sèche sont respectivement dix fois et quatre cents fois supérieures à celles précédemment publiées (CLHP-SM ${ }^{2}$, quantification de la cerbérine par la nériifoline). La quantification de la cerbérine par la nériifoline semble plus pertinente par dosage spectrophotométrique qui élude les différences de fragmentation et de susceptibilités à l'ionisation. La concentration en nériifoline se rapproche davantage de la graine du fruit frais. Les différences observées peuvent néanmoins s'expliquer par une origine géographique différente de la plante et une période de maturation différente.

Il s'agit, à notre connaissance, du premier dosage de la tanghinine et de la déacétyltanghinine dans les graines du C. manghas rapporté. La déacétyltanghinine est le principal cardénolide glycosidique présent dans la graine du faux manguier. Elle ne représente toutefois pas nécessairement la molécule la plus significative sur le plan toxicologique.

\section{P17. Intoxication volontaire avec un pesticide oublié : l'aldicarbe}

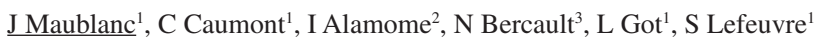
${ }^{I}$ Service de Biochimie, Pharmacologie et Toxicologie, CHR La Source, Orléans; ${ }^{2}$ Service d'hématologie, CHR La Source, Orléans ; ${ }^{3}$ Service de Réanimation Médicale, CHR La Source, Orléans

Objectif : L'aldicarbe est un insecticide de la famille des carbamates dont la distribution et l'utilisation sont interdites en France et en Union européenne depuis 2007 (JORF $n^{\circ} 92$ du 18 avril 2003) en raison de son importante toxicité $\left(\mathrm{DL}_{50}=0,9 \mathrm{mg} / \mathrm{kg}\right.$ chez le rat) (INRS, Fiche technique, 1997). Nous rapportons ici un cas d'intoxication volontaire à ce pesticide oublié :

Un agriculteur retraité de 66 ans, atteint de troubles bipolaires, est retrouvé dans son garage par sa femme et, conscient, il avoue avoir ingéré en quantité inconnue du Temik ${ }^{\circledR}$, spécialité contenant de l'aldicarbe. Il est rapidement pris en charge par le SMUR qui relève un myosis, des sueurs profuses, une $\mathrm{TA}=180 / 110 \mathrm{mmHg}$ et une bradycardie. Suite à l'apparition de troubles de la conscience, le patient est intubé, ventilé et reçoit de l'atropine et du midazolam. Lors de son admission dans le service de réanimation 2 heures après l'ingestion, il est en coma profond et présente une hypothermie $\left(34,6^{\circ} \mathrm{C}\right)$, un érythème du haut du corps et des troubles de la repolarisation à l'ECG. La prise en charge consiste en un traitement symptomatique par atropine, une hyperhydratation intensive et un lavage gastrique. L'évolution est favorable : extubation du patient à $\mathrm{J} 4$ et transfert en Centre Hospitalier Spécialisé à $\mathrm{J} 8$.

Méthode : Au cours du séjour en réanimation, en complément du suivi clinique, des bilans biologiques comprenant l'activité cholinestérasique ont été réalisés. Une mesure des concentrations de l'aldicarbe, a posteriori, ont également permis une documentation de ce cas. Le dosage de l'aldicarbe a été effectué par chromatographie en phase liquide couplée à la spectrométrie de masse en tandem (CL-SM/SM). Un traitement par simple défécation de l'échantillon plasmatique $(100 \mu \mathrm{L})$ avec $250 \mu \mathrm{L}$ d'acétonitrile contenant l'étalon interne (diazépam-d5 à $0,1 \mathrm{mg} / \mathrm{L}$ ) permet une détermination des concentrations de 20 à $2000 \mu \mathrm{g} / \mathrm{L}$ (limite de détection : $1 \mu \mathrm{g} / \mathrm{L}$ ).

Résultats : À l'admission, l'activité cholinestérasique était de 693 UI/L dans le plasma et $4173 \mathrm{UI} / \mathrm{L}$ dans les érythrocytes. Le lendemain, cette activité plasmatique était augmentée 1048 UI/L. Les autres paramètres biologiques sont restés dans les valeurs usuelles. La méthode de dosage de l'aldicarbe a été validée (totalité des CV \% et biais relatif inférieurs à $15 \%$ ) et a permis de mesurer, pour ce patient, les concentrations plasmatiques qui sont rapportées dans le tableau suivant :

\begin{tabular}{|l|c|c|c|c|c|}
\hline Délai après ingestion & $2 \mathrm{~h}$ & $21 \mathrm{~h}$ & $45 \mathrm{~h}$ & $50 \mathrm{~h}$ & $71 \mathrm{~h}$ \\
\hline $\begin{array}{l}\text { Concentration plasmatique } \\
\text { d'aldicarbe }\end{array}$ & $3710 \mu \mathrm{g} / \mathrm{L}$ & $373 \mu \mathrm{g} / \mathrm{L}$ & $<20 \mu \mathrm{g} / \mathrm{L}$ & $<20 \mu \mathrm{g} / \mathrm{L}$ & non détecté \\
\hline
\end{tabular}

Deux métabolites toxiques de l'aldicarbe, l'aldicarbe sulfone et l'aldicarbe sulfoxide, ont été détectés mais non quantifiés dans les quatre premiers prélèvements. Pour ces deux composés, un pic plasmatique est observé 21 heures après l'ingestion.

Conclusion : Ce cas clinique confirme les rares données toxicocinétiques concernant l'aldicarbe: absorption digestive rapide et apparition des symptômes quelques minutes seulement après l'ingestion (Covaci et al., Anal Toxicol. 1999). De plus, la concentration mesurée 2 heures après l'ingestion, est parmi les plus hautes concentrations décrites dans la littérature avec une évolution favorable, démontrant l'intérêt d'une prise en charge rapide de ce type d'intoxication (Lacassie et al., Forensic Sci Int. 2001). Enfin, ce cas rappelle que le retrait du marché d'un produit (pesticides, médicament ou toxique) ne signifie pas sa disparition des foyers et qu'il ne doit donc pas être oublié des urgentistes, réanimateurs et toxicologues.

\section{P18. Intoxication par la romifidine : à propos de 3 cas}

AF Villa $^{1}$, E Saussereau ${ }^{2}$, C Lacroix ${ }^{2}$, R Rubinsztajn ${ }^{3}$, A Cordier $^{1}$, I Blanc ${ }^{1}$, D Chataigner ${ }^{1}$, R Garnier ${ }^{1}$

${ }^{I}$ Centre Antipoison de Paris, Hôpital Fernand Widal, Paris ; ${ }^{2}$ Laboratoire de toxicologie, Groupe hospitalier du Havre, Le Havre ; Réanimation infantile, Hôpital Raymond Poincaré, Garches

Introduction : La romifidine est un sédatif et analgésique pour chevaux administrée chez l'animal par voie intraveineuse. Ce médicament est structurellement et pharmacologiquement proche de la clonidine. À notre connaissance il n'y a pas de cas publié d'intoxication humaine par ce médicament. Nous rapportons trois cas.

Cas $\mathbf{n}^{\circ} 1$ : Une femme de 34 ans s'est administré volontairement $26,3 \mathrm{mg}$ de romifidine $\left(3 \mathrm{~mL}\right.$ Sedivet $\left.{ }^{\circledR}\right)$ par voie intraveineuse. Deux heures plus tard elle était bradycarde (38/min), sa pression artérielle (PA), sa fréquence respiratoire et son état de conscience étaient normaux; sa température corporelle était de $36{ }^{\circ} \mathrm{C}$. Le bilan biologique était sans particularité en dehors d'une discrète élévation de la troponine Ic à $0,31 \mu \mathrm{g} / \mathrm{L}(\mathrm{N}<0,1)$. L'ECG ne montrait pas d'autre anomalie que la bradycardie sinusale. L'échocardiographie cardiaque était normale. L'examen clinique et la troponine se sont spontanément normalisés en 48 heures.

Cas $\mathbf{n}^{\circ} 2$ : Un homme de 27 ans s'est administré par voie sous cutanée, dans un but suicidaire, $2 \mathrm{mg}$ de romifidine $\left(2 \mathrm{~mL}\right.$ Romidys $\left.{ }^{\circledR}\right)$. À H12 il était bradycarde (37/min), sa PA était de $111 / 63 \mathrm{mmHg}$ et sa température corporelle de $36,1^{\circ} \mathrm{C}$. L'ECG non montrait pas d'autre anomalie que la bradycardie sinusale. On lui a administré deux fois $0,5 \mathrm{mg}$ d'atropine par voie intraveineuse à $5 \mathrm{~min}$ d'intervalle et il a été transféré dans le service de réanimation où il est arrivé somnolent et toujours bradycarde (40/min); sa 
température était de $36,8{ }^{\circ} \mathrm{C}$. Le bilan biologique était normal. L'évolution clinique a été favorable en $48 \mathrm{~h}$.

Cas $n^{\circ}$ 3: Un enfant de 4 ans sans antécédent particulier, a ingéré accidentellement $26,3 \mathrm{mg}$ de romifidine $\left(3 \mathrm{~mL}\right.$ Sedivet $\left.^{\circledR}\right)$. Trois heures plus tard il avait un état de conscience fluctuant, alternant des phases de somnolence avec pauses respiratoires et des phases de grande agitation; l'examen clinique montrait aussi un myosis serré bilatéral, une bradycardie (70/min ; $\mathrm{N}:$ 90-105) et une pression artérielle un peu élevée (120/60 mmHg). L'enfant a été sédaté, intubé et ventilé. L'ECG ne montrait pas d'autre anomalie que la bradycardie sinusale ; cette dernière a été maximale (55-60/min) au cours de premières heures d'hospitalisation; elle a été corrigé par l'administration d'atropine. Le bilan biologique a toujours été normal. L'enfant a été extubé à J3. La romifidine a été mesurée par LC MS/MS ; (LOQ : $0,2 \mu \mathrm{g} / \mathrm{L}$ ), à H5, dans le plasma, les urines et le liquide gastrique où ses concentrations étaient respectivement de 13,3 $\mu \mathrm{g} / \mathrm{L}, 166,6 \mu \mathrm{g} / \mathrm{L}, 2452,7 \mu \mathrm{g} / \mathrm{L}$. Les concentrations plasmatiques et urinaires à $\mathrm{H} 26$ n'étaient pas quantifiables.

Discussion : La romifidine est un agent agoniste des récepteurs alpha 2 adrénergique. En inhibant la libération de la noradrénaline, la romifidine exerce une activité sédative et analgésique. Sa demi-vie d'élimination de la romifidine est d'environ 2 heures, chez l'animal. Les effets observés dans ces 3 cas sont proches de ceux décrits avec la clonidine. Les patients intoxiqués ont présenté une des troubles de conscience et une bradycardie; chez l'enfant une dépression respiratoire et un myosis ont également été observés.

$\overline{\text { P19. Évolution du rapport de concentration moelle osseuse/ }}$ sang de sept molécules (morphine, caféine, méprobamate, cyamémazine, citalopram, diazépam et nordazépam) Étude cinétique chez le lapin

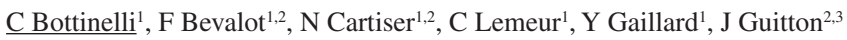
${ }^{1}$ Laboratoire LAT-LUMTOX, Lyon; ${ }^{2}$ Université Lyon 1, Lyon; ${ }^{3}$ Hospices civils de Lyon, Lyon

Objectif : Le but de cette étude était de comparer l'évolution, en fonction du temps, des rapports de concentration moelle osseuse / sang de différentes molécules médicamenteuses. De par son caractère fortement lipidique, la moelle osseuse (MO) peut être un lieu d'accumulation des xénobiotiques (N Cartiser et al., Int J Legal Med. 2010). Afin d'interpréter les résultats d'analyse obtenus sur cette matrice, il est important d'évaluer ce phénomène. Sept molécules aux propriétés différentes, et notamment des $\log \mathrm{P}$ allant de $-0,07$ à 4,53 ont été étudiées.

Méthodes : Deux séries d'expérimentation ont été réalisées : 30 lapins (New Zealand, albinos) ont reçu, par voie intraveineuse, $1 \mathrm{mg} / \mathrm{kg}$ de morphine, $12,5 \mathrm{mg} / \mathrm{kg}$ de caféine, $25 \mathrm{mg} / \mathrm{kg}$ de méprobamate, $3,9 \mathrm{mg} / \mathrm{kg}$ de cyamémazine et ont été sacrifiés après $30 \mathrm{~min}, 1 \mathrm{~h}, 2 \mathrm{~h}, 4 \mathrm{~h}, 7 \mathrm{~h}$ et $17 \mathrm{~h}$ (5 lapins par temps). Vingt-cinq autres lapins ont reçu $2,8 \mathrm{mg} / \mathrm{kg}$ de citalopram et $1,1 \mathrm{mg} / \mathrm{kg}$ de diazépam puis sacrifiés après $2 \mathrm{~h}, 4 \mathrm{~h}, 7 \mathrm{~h}, 17 \mathrm{~h}$ et $32 \mathrm{~h}$ (5 lapins par temps).
La MO et le sang central ont été collectés immédiatement après le sacrifice et analysés par GC-MSMS en utilisant une méthode validée spécifiquement sur chacune des deux matrices. Le métabolite principal du diazépam, le nordazépam, a également été dosé lors des analyses.

Résultats : L'étude du rapport des concentrations MO/sang a permis de comparer la distribution des molécules dans ces deux matrices. Ces rapports (moyenne de $\mathrm{n}=5$ lapins) en fonction du temps (en heures) sont représentés dans les figures ci-dessous; le tracé horizontal représentant sur chaque graphe un rapport MO/Sang $=1$.

Ici, sont représentés les résultats de trois molécules ; A : caféine ( $\log \mathrm{P}-0,07)$, $\mathrm{B}:$ morphine $(\log \mathrm{P} 0,89)$ et $\mathrm{C}$ : diazépam $(\log \mathrm{P} 2,82)$. Les autres molécules ayant un $\log \mathrm{P}>1$ sont la cyamémazine, le nordazépam et le citalopram. Excepté ce dernier, toutes ont un MO/Sang > 1, quel que soit le temps considéré. En ce qui concerne la caféine et le méprobamate $(\log \mathrm{P} 0,7), \mathrm{MO} / \mathrm{Sang}<1$ quel que soit le temps considéré, ce qui est compatible avec les données humaines postmortem précédemment publiées.

Conclusion : D'après ces données, le comportement des molécules testées est de trois types: rapport des concentrations $\mathrm{MO} /$ sang toujours supérieur à 1 , toujours inférieur à 1 ou variable. Le $\log P$ apparait comme un paramètre pouvant prédire l'accumulation dans la MO mais il ne constitue probablement pas le seul paramètre à prendre en compte.

\section{P20. Erreurs médicamenteuses liées au mésusage des dispositifs d'administration des formes liquides orales: estimation de la dose d'exposition}

\section{Muller, E Gomes, P Boltz, C Moulut, J Manel \\ Centre Antipoison et de Toxicovigilance, $\mathrm{CHU}$, Nancy}

Objectif : Les erreurs médicamenteuses liées à une utilisation inappropriée des dispositifs d'administration des médicaments sous forme orale liquide peuvent conduire à des intoxications plus ou moins graves. Actuellement, le centre antipoison du CHU de Nancy est consulté annuellement pour 150 cas. Ce travail a pour objectif d'établir des fiches descriptives des dispositifs de dispensation des formes orales liquides et de proposer un calculateur des quantités de principes actifs effectivement administrés en cas de mésusage : erreur sur le poids ou interversion de dispositifs.

Méthode : Dans une première étape un recensement des médicaments concernés a été établi à partir des situations d'exposition disponibles dans le système d'information des centres antipoison français (Sicap). Des échantillons ont été demandés aux laboratoires concernés et les dispositifs d'administration ont été décrits. Une phase expérimentale de mesure des différents volumes concernés a été réalisée à l'aide d'eau distillée et d'une balance de précision (Denver Instrument S-64). Les spécialités et dispositifs ont été photographiés afin d'illustrer les fiches descriptives par spécialités. Enfin, les informations ont été implémentées dans un calculateur accessible par Internet.
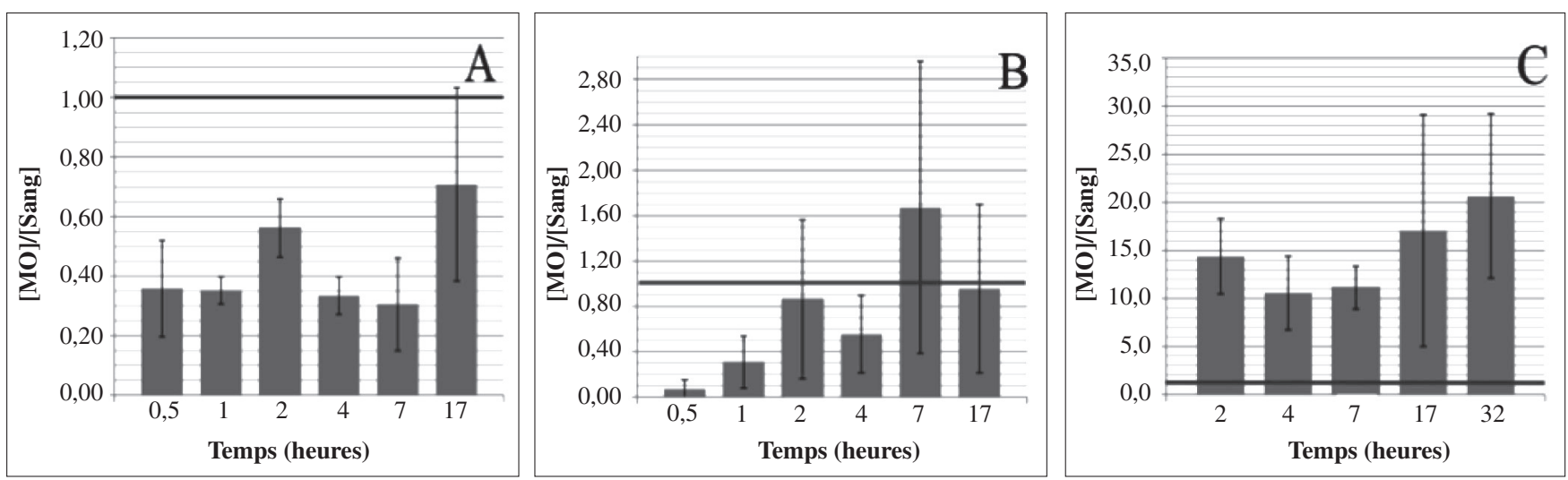
Résultats : Une liste de 59 spécialités a été établie, concernant les classes principalement retrouvées dans ce type d'erreur. Les échantillons de 50 spécialités ont été obtenus 17 antibiotiques, 6 anti-inflammatoires, 4 antiépileptiques, 4 antalgiques, 19 médicaments d'autres classes (diurétiques, antiémétiques, antitussifs...). Pour chacun d'eux, une fiche descriptive standardisée et synthétique du dispositif et de la spécialité est proposée. Les photographies permettent d'échanger précisément avec le demandeur pour approcher précisément la situation d'exposition. Les dispositifs d'administration décrits actuellement sont des pipettes (29) graduées pour la plupart en dose-poids, cuillères (7), gobelets (12) et compte-goutte (2).

Discussion : Les conversions volume - masse avec un volume gradué poids corporel posent au quotidien des problèmes itératifs pour l'évaluation du risque en cas de mésusage ou d'erreur thérapeutique. Les pages web mises à disposition permettre le regroupement des informations utiles et proposent un calculateur permettant la réalisation sans délai des conversions. L'utilisation d'eau distillée permet une approche simplifiée de la mesure des volumes. À défaut de pouvoir disposer d'un recensement exhaustif, ce travail recense les dispositifs qui ont conduit à une erreur thérapeutique.

Conclusion : Reconnaître, préciser, convertir... telles sont les possibilités offertes aux médecins des centres antipoison pour améliorer l'évaluation des risques des expositions aux formes liquides médicamenteuses munies d'un dispositif d'administration. Une extension à l'ensemble des dispositifs d'administration sur le marché devra être mise en œuvre.

\section{P21. Intoxication mortelle par la caféine}

M Evrard ${ }^{1}$, E Desmedt ${ }^{1}$, I Mengin ${ }^{1}$, M Poussel ${ }^{2,3}$, A Kimmoun ${ }^{3,4}$, B Levy ${ }^{3,4}$, $\mathrm{N} \mathrm{Gambier}^{5}$, F Dudek ${ }^{6}$, JF Poussel ${ }^{7}$, B Chenuel ${ }^{2,3}$, E Puskarczyk $^{1}, \mathrm{~J} \mathrm{Manel}^{1}$ ${ }^{1}$ CAPTV, CHU, Nancy; ${ }^{2} E F R, C H U$, Nancy; ${ }^{3}$ Université Lorraine; ${ }^{4}$ Réanimation médicale, Institut Louis Mathieu, CHU, Nancy; ${ }^{5} \mathrm{CRPV}$ de Lorraine, CHU, Nancy; ${ }^{6}$ Service d'accueil des urgences, CHG, Briey; ${ }^{7}$ Réanimation médicale, Hôpital de Mercy, CHR, Metz

Objectif : La caféine (1,3,7-trimethylxanthine, CAS 58-08-2) est la plus méthylée des bases xanthiques et une des substances les plus consommées pour ses propriétés excitantes: boissons, aliments, médicaments, compléments alimentaires et autres « boissons énergisantes » en contiennent. La diminution de la perception de l'effort en fait une molécule de choix des milieux sportifs. Le cas d'une défaillance cardiaque fatale causée par l'ingestion volontaire massive de caféine chez un sujet dépressif amateur de culturisme est rapporté.

Méthodes : Description du cas. Synthèse et discussion bibliographique.

Résultats : Un homme de 44 ans se présente spontanément aux urgences suite à l'ingestion d'une dose massive de caféine anhydre (DSI estimée à $10 \mathrm{~g}$ ) : sueurs, tremblements et tachypnée (TA $123 / 83 \mathrm{mmHg}, \mathrm{FC}=75 / \mathrm{min}$, $\mathrm{S}_{\mathrm{p}} \mathrm{O}_{2}=100 \%$ sans $\mathrm{O}_{2}$ ) rapidement suivis d'une fibrillation ventriculaire $(\mathrm{FV})$ réfractaire à 30 minutes de réanimation. Une ECMO est mise en œuvre 120 minutes après l'arrêt circulatoire initial. Le bilan biologique montre une hyperloceucytose à $14,740 \mathrm{GL}^{-1}, \mathrm{~K}^{+}: 2,6 \mathrm{mEq} . \mathrm{L}^{-1}$, glycémie : 4,02 g.L $\mathrm{L}^{-1}$, CPK : 9040 UI.L ${ }^{-1}$, troponine I : $31,01 \mu \mathrm{g} . \mathrm{L}^{-1}$, CK-MB : 346 UI.L $^{-1}$. Un dosage toxicologique objective des taux sanguins très élevés en caféine : $190,0 \mathrm{mg} \cdot \mathrm{L}^{-1}$ (taux thérapeutiques : $5-20 \mathrm{mg} . \mathrm{L}^{-1}$, toxique $>50 \mathrm{mg} . \mathrm{L}^{-1}$, rapporté létal dès $\left.100 \mathrm{mg} . \mathrm{L}^{-1}\right)$. Malgré une tentative d'épuration par hémofiltration veinoveineuse à haut volume (CVVHF) conjointe à l'ECMO, les concentrations plasmatiques du xénobiotique restent à des niveaux toxiques $\left(145,2 \mathrm{mg} . \mathrm{L}^{-1}\right.$ initialement, $130,1 \mathrm{mg} . \mathrm{L}^{-1}$ après 2 heures de CVVHF). Le patient décède d'un état de choc réfractaire à $\mathrm{H}_{13}$ de l'exposition $\left(\mathrm{H}_{4}\right.$ post ECMO). L'hyperexcitabilité cellulaire induite au niveau du SNC et au niveau cardiaque réalise le risque majeur d'une telle intoxication. L'inhibition compétitive des récepteurs à l'adénosine (A2a notamment) conduit à la majoration de la libération des catécholamines endogènes tandis que l'inhibition des phosphodiestérases cytoplasmiques induit l'accumulation d'AMPc, l'augmentation du calcium intracellulaire avec hypokaliémie de transfert corollaire. L'ensemble conduit à la transduction exacerbée des signaux $\beta 1$ et $\beta 2$ à l'origine du déclenchement, du maintien ou de l'aggravation de troubles du rythme incompatibles avec une hémodynamique efficace. Sédation précoce, utilisation d'un $\beta$-bloqueur à courte durée d'action associé à l'utilisation d'agoniste $\alpha 1$ ou de vasopressine semblent des pistes thérapeutiques utiles, associées à l'administration de charbon activé.

Conclusion: Seules des stratégies thérapeutiques ciblées et précoces permises par la connaissance de la physiopathologie sont susceptibles d'éviter l'issue fatale de telles intoxications massives. Par ailleurs, ce cas pose à nouveau le problème de l'accès du grand public à des quantités létales de substances actives.

\section{$\overline{\text { P22. Quantification de la prégabaline par CLIH-SM à haute }}$ résolution dans des prélèvements post-mortem : à propos de seize cas}

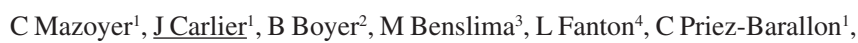
Y Gaillard ${ }^{1}$

${ }^{1}$ Laboratoire LAT-LUMTOX, La Voulte-sur-Rhône ; ${ }^{2}$ Institut de Médecine Légale, Centre Hospitalier Universitaire, Clermont-Ferrand; ${ }^{3}$ Institut de Médecine Légale, Centre Hospitalier Universitaire, Nîmes; ${ }^{4}$ Institut de Médecine Légale, Faculté de médecine, Lyon

Objectif : La prégabaline est un médicament synthétique (Lyrica ${ }^{\circledR}$; Pfizer ; New York, USA) indiqué dans le traitement des épilepsies, des troubles de l'anxiété et des algies neuropathiques. Il existe de rares cas d'intoxication mais dont l'issue fut parfois fatale. Les concentrations en prégabaline dans des prélèvements post-mortem humains et la répartition post-mortem de la molécule demeurent pourtant rarement documentées. Face à une littérature peu prolifique, nous nous proposons de rapporter les concentrations dans les prélèvements autopsiques de seize individus traités par le Lyrica ${ }^{\circledR}$. Nous rapportons entre autres un cas de surdose mortelle mixte impliquant la prégabaline.

Méthodes : Les analyses sont réalisées aux moyens d'une technique originale en chromatographie en phase liquide d'interaction hydrophobe (CLIH) couplée à une détection par spectrométrie de masse (SM) à haute résolution (technologie orbitrap) $(\mathrm{m} / z, 160,1334 \pm 5 \mathrm{ppm})$. L'identification de la prégabaline est confirmée par son spectre de fragmentation $\left(\mathrm{CLIH}-\mathrm{SM}^{2}\right)$. La préparation de l'échantillon consiste en une simple précipitation des protéines dans l'acétonitrile.

Résultats : La méthode a été validée dans le sang total avec des CV inférieurs à $15 \%$. La réponse est linéaire pour des concentrations allant de 1 à $200 \mu \mathrm{g} \cdot \mathrm{mL}^{-1}$ pour des limites de détection et de quantification s'élevant respectivement à 0,025 et $0,060 \mu \mathrm{g} \cdot \mathrm{mL}^{-1}$.

Les concentrations mesurées dans les prélèvements des individus sont résumées dans le tableau ci-dessous :

\begin{tabular}{|c|c|c|c|c|c|c|}
\hline Matrice & $\begin{array}{c}\text { Sang } \\
\text { périphérique } \\
\left(\mu \mathrm{gg} \cdot \mathrm{mL}^{-1}\right)\end{array}$ & $\begin{array}{c}\text { Sang } \\
\text { cardiaque } \\
\left(\mu \mathrm{g} \cdot \mathrm{mL}^{-1}\right)\end{array}$ & $\begin{array}{c}\text { Urine } \\
\left(\mu \mathrm{g} \cdot \mathrm{mL}^{-1}\right)\end{array}$ & $\begin{array}{c}\text { Bile } \\
\left(\mu \mathrm{g} \cdot \mathrm{mL}^{-1}\right)\end{array}$ & $\begin{array}{c}\text { Rein } \\
\left(\mu \mathrm{g} \cdot \mathrm{g}^{-1}\right)\end{array}$ & $\begin{array}{c}\text { Foie } \\
\left(\mu \mathrm{g} \cdot \mathrm{g}^{-1}\right)\end{array}$ \\
\hline Médiane & 5,6 & 4,6 & 574,7 & 18,2 & 14,0 & 4,9 \\
\hline Moyenne & 21,7 & 9,6 & 897,4 & 21,6 & 20,9 & 6,4 \\
\hline $\begin{array}{l}\text { Valeurs } \\
\text { extrêmes }\end{array}$ & 1,1 à 206,7 & 1,5 à 54,1 & 126,6 à 2004,6 & 12,2 à 58,3 & 11,1 à 44,5 & 3,2 à 12,5 \\
\hline
\end{tabular}

Conclusion : La CLIH est particulièrement adaptée à la prégabaline compte tenu de la polarité et de la faible masse de la molécule. L'extraction par précipitation est rapide, simple à mettre en œuvre et présente l'échantillon dans un solvant composé de $90 \%$ d'acétonitrile et $10 \%$ d'eau, conditions adéquates pour l'injection. La détection à haute résolution permet de réaliser une quantification hautement spécifique en mode SIM (selected ion monitoring) ( $\pm 5 \mathrm{ppm}$ ) qui permet de s'affranchir des méthodes de détection classiques en $\mathrm{SM}^{2}$ inadaptées à la prégabaline (transitions non spécifiques). 
Cette méthode nous a permis de mesurer les concentrations dans des prélèvements post-mortem de seize individus, fournissant la première base de données sur la répartition post-mortem du médicament. Nous rapportons parmi ces personnes, un cas d'intoxication mortelle par surdose mixte impliquant la prégabaline. Il s'agit, à notre connaissance, de la concentration sanguine la plus élevée jamais présentée $\left(206,7 \mu \mathrm{g} \cdot \mathrm{mL}^{-1}\right)$.

\section{P23. Étude de la dégradation bactérienne du clonazépam dans le lait de vache : application à un cas médico-légal}

\section{T Besnard, M Nicolas}

\section{Laboratoire LTB, Narbonne}

Objectif : À la suite d'un acte criminel dans une maison de retraite, cinq personnes âgées ont été intoxiquées par du Rivotril ${ }^{\circledast}$ mis dans un broc de lait. Une expertise toxicologique du lait contenu dans le broc nous a été confiée 6 mois après les faits. Les concentrations trouvées en clonazépam dans le lait étaient très faibles et n'étaient pas compatibles avec la survenue des symptômes évoqués lors des intoxications. Nous avons donc émis l'hypothèse d'une dégradation bactérienne du clonazépam au cours du temps dans cette matrice comme cela avait déjà pu être évoqué avec la transformation du flunitrazépam en 7-aminoflunitrazépam par des nitroréductases bactériennes. Nous avons étudié, après isolement de deux souches bactériennes, la dégradation du clonazépam sur un mois à différentes températures et remonter ainsi à la concentration initiale contenue dans le lait.

Méthodes : Les bactéries contenues dans le lait ont été isolées par la technique de MALDI-TOF-MS. Après isolement de deux souches bactériennes et mise en culture, différents aliquots de lait ont été surchargés avec une quantité donnée de clonazépam. Une série d'aliquots a été ensemencée stérilement avec une souche, une autre série avec la deuxième souche et une troisième série avec un mélange à part égale des deux souches. Les dosages du clonazépam et du 7-aminoclonazépam dans le lait ont été réalisés par CLHP-SM/SM. Il s'agissait d'une injection directe après une dilution des échantillons au $1 / 200^{\mathrm{e}}$ dans de l'eau. La séparation chromatographique a été réalisée sur colonne HSS C18 $150 \times 2,1 \mathrm{~mm}$ et la phase mobile était constituée d'un mélange acétonitrile/formiate d'ammonium à $\mathrm{pH}=3$. Le mode MRM a été utilisé pour suivre les transitions 316,1 > 270,1 et 286,1 > 250,1 respectivement pour le clonazépam et le 7-aminoclonazépam. Le clonazépam-d4 et le 7-aminoclonazépam-d4 ont été utilisés comme étalons internes. La méthode a été validée selon les critères habituels. Les séries d'aliquots ont été analysées à différentes températures $\left(-20^{\circ} \mathrm{C},+5^{\circ} \mathrm{C}\right.$ et $+20^{\circ} \mathrm{C}$ ) sur un mois.

Résultats : La méthode de dosage du clonazépam et du 7-aminoclonazépam dans le lait de vache a été validée. Les LOD étaient de 5 et $3 \mathrm{ng} / \mathrm{mL}$ respectivement pour le clonazépam et le 7-aminoclonazépam. Les LOQ étaient de 10 et $7 \mathrm{ng} / \mathrm{mL}$ respectivement pour le clonazépam et le 7-aminoclonazépam. La répétabilité et la reproductibilité ont montré des $\mathrm{CV}<15 \%$. Micrococcus luteus et Enterrococcus faecium, deux bactéries nitroréductases, ont été isolées du lait contenu dans le broc. Nous avons observé à $\mathrm{J} 30$ une dégradation du clonazépam de $+45 \%$ à $+20{ }^{\circ} \mathrm{C}$ pour Enterococcus faecium, $+79 \%$ à $+20{ }^{\circ} \mathrm{C}$ pour Micrococcus luteus et $50 \%$ à $+20^{\circ} \mathrm{C}$ pour le mélange des deux bactéries. Pas de dégradation du clonazépam à $-20^{\circ} \mathrm{C}$ à $\mathrm{J} 30$. Une dégradation de $<1 \%$ est apparue à $\mathrm{J} 25$ et à $+5{ }^{\circ} \mathrm{C}$ pour les deux bactéries pris isolement et combinées. En fonction du rapport clonazépam/7-aminoclonazépam et en tenant compte du pourcentage de dégradation des deux bactéries mélangées, nous avons pu établir une concentration de $1,54 \mu \mathrm{g} / \mathrm{mL}$ de lait de clonazépam au moment des faits.

Conclusion : Notre étude a montré que deux bactéries, Micrococcus luteus et Enterococcus faecium, deux nitroréductases, étaient responsables de la dégradation importante du clonazépam dans le lait à température ambiante (79\% M. luteus vs $45 \%$ E. faecium). La concentration de $1,54 \mu \mathrm{g} / \mathrm{mL}$ de lait de clonazépam trouvée pouvait correspondre à l'introduction criminelle approximative de $2 \mathrm{~mL}$ de clonazépam en solution buvable dans le broc de lait de 3 litres.

\section{P24. Évaluation de l'effet matrice lors de l'analyse de stupéfiants par LC-MS/MS}

\author{
M Deville $^{1}$, N Dubois ${ }^{1}, \mathrm{C}$ Pirard $^{1}$, C Charlier ${ }^{1,2}$
}

${ }^{1}$ Service de Toxicologie Clinique, Médico-légale, de l'Environnement et en Entreprise, Centre Hospitalier Universitaire, Liège, Belgique ; ${ }^{2}$ Centre Interfacultaire de Recherche du Médicament, Université de Liège, Belgique Objectif : La chromatographie liquide couplée à la spectrométrie de masse (LC-MS) est une technique de choix pour le dosage de diverses substances dans les liquides biologiques. Cependant, ces milieux sont susceptibles de contenir des composés qui vont coéluer avec les analytes d'intérêt et altérer leur ionisation (amplification ou suppression d'ionisation). Ce phénomène, appelé « effet matrice » $(\mathrm{EM})$, peut compromettre la fiabilité des résultats. Il doit donc faire partie intégrante du processus de validation. Le but du présent travail est d'évaluer, au moyen de deux approches distinctes, l'importance de l'EM éventuellement présent lors du dosage des cannabinoïdes dans le sérum (THC, THC-OH et THC-COOH) et la salive (THC), prétraîtés par extraction liquide-liquide. D’autres stupéfiants (cocaïne, opiacés, amphétamines) sériques et salivaires ont également été soumis à la même procédure.

Méthodes : La première méthode d'évaluation de l'EM est l'addition post extraction (Matuszewski B.K. et al., Anal Chem. 2003). Elle consiste à comparer l'intensité du signal obtenu en LC-MS d'une même quantité de composé en présence et en l'absence de matrice extraite. Le résultat est exprimé en termes de pourcentage par rapport au signal obtenu sans matrice. La deuxième approche est l'infusion post colonne. Elle consiste à observer la variation du signal lorsque les composés sont introduits directement dans le spectromètre de masse pendant qu'un échantillon biologique extrait est élué de la colonne chromatographique (Bonfiglio R et al., Rapid Commun Mass Spectrom. 1999). Lors de cette étude, l'addition post extraction et l'infusion post colonne ont été réalisées sur 20 échantillons sériques et 15 échantillons salivaires. Les analyses ont été effectuées sur un système UPLC Waters Acquity couplé à un spectromètre de masse Quattro Premier triple quadripôle utilisant une source ESI. Le contrôle de l'instrument et le traitement des données ont été effectués à l'aide du logiciel Masslynx 4.1.

Résultats : Lors de l'analyse du THC, l'EM dans les échantillons de sérum diminue l'intensité du signal de 4 à $78 \%$ suivant l'extrait analysé (valeur moyenne de $19 \%$ ). Cette diminution du signal correspond à une supression d'ionisation. Celle-ci est moins importante pour le THC-COOH qui subit un effet moyen de $7 \%$. Le THC-OH est le seul composé qui connait, dans les échantillons sériques, une augmentation du processus d'ionisation allant de 8 à $44 \%$ ( $25 \%$ en moyenne). Ce renforcement du processus d'ionisation en ESI est un phénomène plutôt rare. Quel que soit l'EM observé et quel que soit le composé analysé, l'intensité de cet effet varie d'un échantillon à l'autre. La suppression d'ionisation pour le THC est plus importante dans le sérum $(19 \%)$ que dans la salive $(7 \%)$.

L'ajout de standards internes deutérés appropriés permet de corriger l'EM observé dans chacun des deux milieux étudiés. En effet, ils subissent la même variation de signal que les analytes correspondants et, dès lors, le rapport de chacun des composés sur son standard interne reste constant quel que soit l'EM. Leur utilisation est donc indispensable pour une quantification fiable de chaque composé. L'infusion post-colonne confirme les EM calculés par la méthode de l'addition post-extraction. Cependant, seuls les EM significatifs entraînent une variation de signal clairement objectivable. Enfin, l'EM observé lors du dosage de divers autres stupéfiants dans le sérum et la salive, a été mesuré par la même approche.

Conclusion : L'effet matrice est un phénomène à la fois composé- et matrice dépendant qui est corrigé de manière adéquate par l'usage de standards internes deutérés, indispensables pour garantir la fiabilité des résultats. 


\section{$\overline{\text { P25. Intoxication aiguë au nébivolol : dosage plasmatique et }}$ suivi d'intoxication par LC-MS/MS}

$\underline{\text { M Soichot }}^{1}$, E Bourgogne $^{1,3}$, B Megarbane ${ }^{2}$, O Laprevote $^{1,3}$

${ }^{1}$ Laboratoire de Toxicologie Biologique, Groupe Hospitalier Saint Louis Lariboisière, AP-HP; 'Réanimation Médicale Toxicologique, Groupe Hospitalier Saint Louis Lariboisière, AP-HP; ${ }^{3}$ Laboratoire Chimie Toxicologie Analytique Cellulaire, EA 4463, Faculté de Pharmacie, Université Paris Descartes, Sorbonne Paris Cité

Introduction : Un homme de 55 ans est admis aux urgences dans un contexte d'intoxication médicamenteuse aiguë volontaire survenue à la suite de conflits familiaux. À l'interrogatoire, le patient dit avoir pris 48 heures plus tôt 30 comprimés de nébivolol $5 \mathrm{mg}^{\circledR}(150 \mathrm{mg}), 20$ comprimés de tétrazépam $50 \mathrm{mg}^{\circledR}(1 \mathrm{~g})$ et 30 comprimés d'amlodipine $5 \mathrm{mg}^{\circledR}(150 \mathrm{mg})$. À l'admission, le patient est conscient, mais asthénique et essoufflé ; son état clinique se dégrade rapidement, avec une hypotension $(87 / 58 \mathrm{~mm} \mathrm{Hg}$ ) et une bradycardie (40/min) accompagnées de marbrures. Le patient est alors mis sous Isuprel ${ }^{\oplus}$ et est pris en charge dans le service de Réanimation Médicale et Toxicologique (RMT). Des prélèvements sanguins et urinaires sont réalisés et envoyés au service de Toxicologie.

Analyses toxicologiques : À l'admission en réanimation, le screening sanguin révèle la présence d'hydroxyzine (concentration estimée à $0,66 \mathrm{mg} / \mathrm{L}$ ), de tétrazépam, d'amlodipine et de nébivolol; le screening urinaire confirme la prise de benzodiazépines. Pour évaluer les concentrations plasmatiques de nébivolol chez le patient et suivre sa cinétique d'élimination, une méthode de dosage a été développée en chromatographie liquide couplée à la spectrométrie de masse en tandem (LC-MS/MS). Dans un tube Eppendorf ${ }^{\boxplus}$ de $1,5 \mathrm{~mL}, 150 \mu \mathrm{L}$ de sérum sont mélangés avec $20 \mu \mathrm{L}$ de colchicine-D3 (standard interne à $100 \mathrm{ng} / \mathrm{mL}$ ) et $150 \mu \mathrm{L}$ de sulfate de zinc $0,14 \mathrm{~mol} / \mathrm{L}$. Après une brève agitation au vortex, le mélange est centrifugé 10 min à $4{ }^{\circ} \mathrm{C}(14000 \mathrm{rpm})$, puis $30 \mu \mathrm{L}$ de surnageant sont injectés dans un système HPLC Aria TLX-1 (Thermo Scientific). La séparation est réalisée sur une colonne Hypersil Gold $100 \times 2,1 \mathrm{~mm} \mathrm{2,1 \mu \textrm {m }}$ (Thermo Scientific) avec un gradient basse pression eau/méthanol ajusté à $\mathrm{pH} 3$ avec $0,1 \%$ d'acide formique à un débit de $0,6 \mathrm{~mL} / \mathrm{min}$. La détection par spectrométrie de masse est réalisée sur un triple quadripôle TSQ Quantum Ultra (Thermo Scientific) opérant à résolution unitaire en mode electrospray positif. Les transitions SRM (Selected Reaction Monitoring) utilisées sont les suivantes : $m / z, 406,3 \rightarrow 151,1$ pour l'identification et la quantification du nébivolol, $m / z, 406,3 \rightarrow 123,1$ pour sa confirmation et $m / z$ 403,2 $\rightarrow 359,2$ pour la colchicine-D3. Le temps d'analyse total est de 12 minutes.

Résultats : Les temps de rétention du nébivolol et de la colchicine-D3 sont respectivement de 7,80 min et de 7,28 $\mathrm{min}$. Une gamme de concentration a été réalisée entre $1 \mathrm{ng} / \mathrm{mL}$ (LLOQ) et $100 \mathrm{ng} / \mathrm{mL}$, avec une linéarité excellente sur 7 points entre la LLOQ et $100 \mathrm{ng} / \mathrm{mL}\left(\mathrm{r}^{2}>0,994\right)$. Le nébivolol a été identifié et quantifié dans le sérum du patient tout au long de sa prise en charge par le service de RMT. Sur les sept différents prélèvements la cinétique d'élimination du nébivolol a été suivie avec des concentrations retrouvées entre 58,9 et $3,2 \mathrm{ng} / \mathrm{mL}$ (concentrations plasmatiques estimées 3 heures après une prise unique de $5 \mathrm{mg}$ de nébivolol : 0,19 à $11,59 \mathrm{ng} / \mathrm{mL}$ (Lefevre et al., Br J Clin Pharmacol. 2007)).

Discussion et Conclusion : À notre connaissance, aucun cas d'intoxication aiguë au nébivolol n'a été décrit à ce jour dans la littérature. À la suite d'une intoxication polymédicamenteuse volontaire massive, l'analyse par LC-MS/ MS a permis d'identifier, de quantifier et de suivre la cinétique d'élimination de ce béta-bloquant. Il est intéressant de noter que la prise en charge du patient a été tardive, soit 48 heures après l'intoxication, et que son état s'est réellement dégradé au moment de son admission aux urgences. Après 5 jours d'hospitalisation en réanimation, le patient, traité par Isuprel ${ }^{\circledR}$ et soumis à une surveillance étroite de ses paramètres vitaux, évoluera favorablement ; il sera ensuite pris en charge dans un service de psychiatrie. $\overline{\text { P26. Simultaneous detection and quantification of } 15 \text { drugs }}$ of abuse in whole blood by online solid-phase extraction and LC-MS/MS

H Grison-Hernando ${ }^{1}$, C Renaud ${ }^{1}$, A Morla ${ }^{2}$, G Deslandes ${ }^{1}$, A Pineau ${ }^{1}$, E Dailly $^{1}$, P Jolliet ${ }^{1}, C_{\text {Monteil-Ganiere }}^{1}$

${ }^{l}$ Laboratoire de Pharmacologie-Toxicologie, $\mathrm{CHU}$ de Nantes ; ${ }^{2} \mathrm{AB}$ Sciex, Les Ulis

Objective: A new quantitation method of drugs of abuse in whole blood was developed and validated with a simple sample pretreatment, online solid-phase extraction and liquid chromatographytandem mass spectrometry (LC-MS/MS): 15 drugs of abuse and metabolites (amphetamine, metamphetamine, MDMA, MDA, MDEA, MBDB, mephedrone, 6-MAM, morphine, codeine, dihydrocodeine, ethylmorphine, cocaine, BEG and cocaethylene) were measured in a single chromatographic run.

Method: For quantitative determination, 14 deuterated analogues were used as internal standards. After protein precipitation of $250 \mu \mathrm{L}$ whole blood with $\mathrm{ZnSO}_{4}$-methanol spiked with a mixture of internal standards, samples were mixed and centrifuged; the supernatant was evaporated to dryness, and reconstituted with mobile phase, before injection in the chromatographic system. First, analytes were loaded on the extraction column (Strata-X, Phenomenex) with a $2 \mathrm{mM}$ ammonium formate buffer. Then, switching the valve entailed the back-flush elution and the passage on the analytical column (Kinetex PFP, Phenomenex) kept at $60{ }^{\circ} \mathrm{C}$. The compounds were well separated in a total run time of $15 \mathrm{~min}$. A gradient was performed with the aqueous phase $\mathrm{A}$ ( $2 \mathrm{mM}$ ammonium formate and $0.2 \%$ formic acid), and the organic phase B ( $2 \mathrm{mM}$ ammonium formate with methanol-acetonitrile (70-30) and $0.2 \%$ formic acid). The mass spectrometer was an API 3200 QTrap $^{\circledast}$ (AB Sciex). The method was developed in scheduled multiple reaction monitoring mode (sMRM), with two transitions per compound. All analytes were detected in positive ionisation mode. The method was validated according to the Cofrac (French Accreditation Committee) document SH GTA 04, and compared to the liquid-liquid extraction-gas chromatography-mass spectrometry (GC-MS) reference method.

Results: The method validation showed a recovery of the precipitation step over $75 \%$. No matrix effect was detected by post-column infusion and by analysis of six blood samples spiked with two different concentrations (relative standard deviation $<15 \%$ ). Intra-day and inter-day precisions, expressed as relative standard deviation, were lower than $15 \%$. Accuracy was higher than $85 \%$. Calibration curves were performed from 10 to $200 \mu \mathrm{g} / \mathrm{L}$ for most analytes with a LOQ of $5.0 \mu \mathrm{g} / \mathrm{L}$, and from 2.5 to $50 \mu \mathrm{g} / \mathrm{L}$ for $6-M A M$ and cocaine with a LOQ of $1.25 \mu \mathrm{g} / \mathrm{L}$. The method was linear with $r>0.995$. The linearity was verified up to $1000 \mu \mathrm{g} / \mathrm{L}$ and $250 \mu \mathrm{g} / \mathrm{L}$, respectively for each calibration range. The results comparison with the GC-MS method showed a good correlation $\left(r^{2}>0.9\right)$.

Conclusion: This method presented sensitivity, accuracy, precision and linearity at least equivalent to those obtained with the GC-MS method. Benefits were multiple: low sample volume, highly reduced time of sample preparation, short chromatography and simultaneous analysis of basic drugs of abuse measured in forensic toxicology, as well as a cathinone compound. It was completely validated and used in routine for drugs of abuse road testing. The present method is the first published measuring simultaneously amphetaminic, cocainic, opiates and mephedrone in whole blood, using online extraction and LC-MS/MS. A close published technique, using online extraction, measured 14 basic drugs of abuse in serum (Ferreiros N et al., Anal Bioanal Chem. 2009). In France, this technique was not available for drugs of abuse road testing, where whole blood was analysed. 
$\overline{\text { P27. Drug facilitated sexual assault by use of ketamine and }}$ diazepam by a gynaecologist

V Di Fazio, SMR Wille, N Samyn

Laboratory of Toxicology, National Institute of Criminalistics and Criminology, Brussels, Belgium

Objective: A DFSA case in a medical setting is discussed; a young woman of 29 years old, only living shortly in Belgium, asked her gynaecologist to place an intra-uterine device as contraception. During the medical procedure, the physician injected a substance ostensibly to reduce pain. She lost her conscience for a while and when she regained it, she felt a second injection from which she collapsed again. When she awoke she noticed that the gynaecologist was touching her intimately. The doctor was very nervous when he noticed that she was awake and he refused her to use her cell phone. She started vomiting and only after he had cleaned up the office, he let her contact her boyfriend. While the doctor insisted that she stayed for observation, the couple left the medical center about an hour after the young women had entered. The next morning, the victim went to the police to press charges for sexual assault.

Method: The Sexual Aggression Set (SAS) used has been developed by our institute and contained evidence seals for genetic analysis, a urine sample and two $5 \mathrm{~mL}$ blood tubes with sodium fluoride and potassium oxalate as anticoagulant were provided around 30 hours after the alleged facts. Our analytical strategy for DFSA cases includes immunological semi-quantitative urine screening for DOA but also, chromatographic screening methods by GC-MS with automated mass spectrometry deconvolution and identification system (AMDIS) and HPLC/DAD for blood and urine. However, to detect compounds at their concentration level a few hours to several days after a single administration, very sensitive and specific target techniques by HPLC or UPLC-MS/MS, GC-MS or GC-FID have been used. In addition, all methods have been validated according to ISO17025 guidelines; several methods have been published by our research group.

Results: The physical examination of the victim revealed the presence of two injection marks on the arm fold of the victim. In this particular case, the evidence for genetic analysis was stored without further analysis since the declarations of the victim did not indicate that sexual contact had occurred. Concerning the toxicological analysis, nordiazepam, temazepam, ketamine and norketamine were detected in urine, while the blood concentrations 30 hours after the alleged facts were as follows: $28 \mathrm{ng} / \mathrm{mL}$ diazepam, $8 \mathrm{ng} / \mathrm{mL}$ nordiazepam, $2 \mathrm{ng} / \mathrm{mL}$ temazepam, $2 \mathrm{ng} / \mathrm{mL}$ ketamine and $6 \mathrm{ng} / \mathrm{mL}$ norketamine. The dosage and the associated effects at the time of the alleged assault were estimated via back-calculations and literature data as required by the prosecutor.

Conclusion: In this case, the toxicological data have contributed to the investigation, by supporting the declarations of the victim and the observations during the physical examination. It is clear that the side effect of irrational behavior and hallucinations due to ketamine can make it difficult for the investigators to evaluate the testimony of the victim. Moreover, ketamine is an illicit substance that is also abused. Analysis of a hair sample would have been of interest to distinguish between a naïve usage and a chronic presumably illicit drug use. Collection of a hair sample was proposed to the judicial authority but unfortunately, refused by the victim. However, the use of ketamine in Belgium is illegal if not performed in a hospital environment.
P28. Stratégies de préparation d'échantillon en ligne : flexibilité du système ARIA ${ }^{\circledR}$ (flux turbulent) en vue de l'analyse rapide de composés d'intérêt clinique dans des matrices biologiques par chromatographie liquide couplée à la spectrométrie de masse (LC-MS/MS)

E Bourgogne $^{1,2}$, M Soichot $^{1}$, C Latour ${ }^{1}$, O Laprevote $^{1,2}$

${ }^{1}$ Laboratoire de Toxicologie Biologique, Groupe Hospitalier Saint Louis Lariboisière, AP-HP; ${ }^{2}$ Laboratoire Chimie Toxicologie Analytique Cellulaire, EA 4463, Faculté de Pharmacie, Université Paris Descartes, Sorbonne Paris Cité

Objectif : La nécessité d'élaborer des stratégies génériques pour cribler rapidement et/ou quantifier de nombreux composés dans les laboratoires de toxicologie biologique demeure un défi. Aujourd'hui, les stratégies modernes de bioanalyse vont de la simple dilution à un prétraitement des échantillons complexes, comme l'extraction en ligne en phase solide (SPE) ou l'extraction liquide-liquide (LLE). Pour accélérer le développement de méthodes et leur validation, les approches génériques à injection directe de fluides biologiques sont hautement souhaitables. Le système de flux turbulent ARIA MX1 (ThermoFisher), un dispositif de permutation de colonne (column-switching) a été évalué comme un système polyvalent pour la recherche ou le dosage de différents composés d'intérêt actuellement dosés au laboratoire de toxicologie. Les objectifs de ce travail sont de présenter, à travers différents exemples (dosage sanguin de cocaïne et opiacés, dosage sanguin de colchicine ou du nébivolol, recherche de LSD ou différenciation des opiacés naturels urinaire), l'avantage d'utiliser le même système de flux turbulent, comme un dispositif de préparation de l'échantillon soit en ligne, soit hors ligne.

Méthodes : Dans le cadre de dosages plasmatiques, après précipitation des protéines par une solution de sulfate de Zinc 0,2 M et ajout d'étalon interne deutéré approprié, les surnageants (10-30 $\mathrm{L}$ ) ont été injectés sur le système d'extraction en ligne pour le dosage de colchicine et cocaïne/opiacés, ou hors ligne pour le dosage du nébivolol. Une séparation chromatographique à l'aide de gradient méthanol+ $0,1 \%$ acide formique/eau $+0,1 \%$ acide formique sur colonne microbore $(50 / 100 \times 2.1 \mathrm{~mm})$ et une détection par spectrométrie de masse en mode tandem en electrospray positif (Thermo Quantum Ultra, ThermoFischer, USA) ont permis d'identifier et de quantifier ces composés. Dans le cadre de recherche urinaire, une étape préalable de déconjugaison ( $\beta$-glucuronidase Patella vulgata) a été effectuée pour améliorer la sensibilité des méthodes.

Résultats et Conclusion : L'utilisation de ce système permet dès à présent de suivre dans le plasma de patients l'évolution de différentes intoxications comme la colchicine entre 0,3 et $15 \mathrm{ng} / \mathrm{mL}$, le nébivolol entre 1 et $100 \mathrm{ng} / \mathrm{mL}$, la cocaïne et ses métabolites méthylecgonine et benzoylecgonine, les opiacés (morphine, codéine, dihydrocodéine et 6 Mono Acétyl Morphine - 6-MAM) entre 1 et $1000 \mathrm{ng} / \mathrm{mL}$. Une confirmation par spectrométrie de masse permet également d'identifier les opiacés urinaires (morphine, normorphine, hydromorphone, codéine, norcodéine, codéthyline, dihydrocodéine, pholcodine, oxycodone, 6-MAM) détectés positivement par immunochimie. Cet instrument peut donc être utilisé comme un appareil générique, capable d'effectuer diverses stratégies en ligne ou hors ligne, adapté au contexte de toxicologie hospitalière (i.e. grand nombre d'analyses différentes de petites séries- ca 20- d'échantillons). Sa facilité d'utilisation pour changer les colonnes de trappage de différentes chimies complète les outils du système ARIA ${ }^{\circledR}$ pour faire face à tous les analytes pour le développement de méthode générique et pour la quantification de petites molécules dans les liquides biologiques. Une stratégie adaptée sera choisie par l'analyste en fonction des propriétés physico-chimiques du composé d'intérêt, de la sensibilité de la méthode nécessaire ainsi que du débit d'analyse souhaité. 